\title{
Deformação harmônica da triangulação de Delaunay
}

\author{
Rafael de Mattos Grisi
}

TESE APRESENTADA

AO

Instituto de Matemática e Estatística

DA

Universidade De SÃo PaUlo

PARA

OBTENÇÃO DO TÍTULO

$\mathrm{DE}$

Doutor EM CIÊNCIAS

\author{
Área de Concentração: Estatística \\ Orientador: Prof. Dr. Pablo Augusto Ferrari
}

Durante o desenvolvimento deste trabalho o autor recebeu auxílio financeiro da CAPES/FAPESP

São Paulo, 28 de agosto de 2009 


\title{
Deformação harmônica da triangulação de Delaunay
}

\author{
Este exemplar corresponde à redação \\ final da tese devidamente corrigida \\ e defendida por Rafael de Mattos Grisi \\ e aprovada pela Comissão Julgadora.
}

Banca Examinadora:

- Prof. Dr. Pablo Augusto Ferrari (orientador) - IME-USP.

- Prof. Dr. Luiz Renato Gonçalves Fontes - IME-USP.

- Prof. Dr. Domingos Humberto Urbano Marchetti - IF-USP.

- Prof. Dr. Pablo José Groisman - UBA.

- Prof. Dr. Roberto Imbuzeiro Moraes Felinto de Oliveira - IMPA. 


\section{Agradecimentos}

Gostaria de começar prestando homenagem a duas pessoas sem as quais este trabalho certamente não haveria tomado forma. Primeiro, sem a ajuda, paciência e orientação de Pablo Ferrari, nada disso teria sido possível. Parafraseando Sebastián Grynberg, "entre el agradecimiento que merece y este que le ofrezco media un desproporción que no desconozco". Em seguida, meu sincero agradecimento ao "quase co-orientador" Pablo Groisman, cujas idéias e incentivo foram essenciais durante todo o processo. Isto sem mencionar sua hospitalidade nas inúmeras visitas que fiz a Buenos Aires durante a realização deste trabalho.

Ainda no âmbito acadêmico, gostaria de agradecer a todos os professores do grupo de probabilidade do IME, especialmente aos professores Antonio Galves, Fábio Machado, Luiz Renato Fontes, Eduardo Jordão e Serguei Popov, pelos ensinamentos e oportunidades que me proporcionaram ao longo destes quatro anos de trabalho.

Não posso deixar de fora todos os meus colegas do IME que, além das muitas e frutíferas discussões acadêmicas, também me proporcionaram diversos momentos de relaxamento e descontração. Em especial, segue o meu muito obrigado a Alexsandro Gallo, Cristian Coletti, Christophe Gallesco, Daniel Takahashi, Pablo Rodriguez, Florência Leonardi, Alexandre Leichsenring, Paulo Lima, Leonardo Rolla, Marcus Marrocos, Jhames Sampaio, Natalia Viana, Sandra Zapata, Paula Cadavid, Gisela Tunes e Artur Lemonte.

Boa parte deste trabalho foi realizado em solo Argentino, segue então o meu agradecimento a todos aqueles com quem tive a felicidade de conviver no período de minha estada. Em especial, e pelos mais diversos motivos, gostaria de agradecer a Inés Armendáriz, Mariela Sued, Maria Eugênia (Maru), Julián Martinez, Sebastián Grynberg, Analia Ferrari e Anaclara Martin.

Passando agora a um foro mais íntimo, como não poderia deixar de ser, agradeço aos meus pais, Léo e Cecília, e meus irmãos, Léo e Mara, pela compreensão, apoio, auxílio e tudo o mais que me proporcionaram, não só no decorrer deste trabalho, mas ao longo de toda vida.

Merecem um agradeciemto especial o meu tio Celso e tias Sandra e Marília, não apenas pela pronta acolhida quando da minha chegada em São Paulo, mas também por todo o suporte e auxílio 
que me prestaram ao longo destes anos. Estarei eternamente em dívida com todos.

Para finalizar gostaria de agradecer especialmente a Ingrid Ramos da Silva, com quem tenho compartilhado minha vida há mais de 5 anos. É ela, sem dúvida, minha maior incentivadora. Foi ela quem me apoiou nos momentos mais turbulentos destes anos, e foi este apoio que me permitiu chegar ao final deste trabalho. 


\section{Resumo}

Dada um processo de Poisson em $\mathbb{R}^{d}$, construímos funções harmônicas na triangulação de Delaunay associada, com comportamento assintótico linear, como limite de um processo de harness sem ruído. Tais funções permitem que construamos uma nova imersão da triangulação de Delaunay em $\mathbb{R}^{d}$, que denominaremos de deformação harmônica.

Palavras-chave: processo pontual ergódico, processo de Poisson, processo de Harness, ladrilhamento de Voronoi, triangulação de Delaunay, medidas de Palm, teorema ergódico. 


\section{Abstract}

Given a Poisson point process in $\mathbb{R}^{d}$, we construct harmonic functions on the associated Delaunay triangulation, with linear assymptotic behaviour, as the limit of a noiseless harness process. These mappings allow us to find a new embedding for the Delaunay triangulation in $\mathbb{R}^{d}$. We call it harmonic deformation of the graph.

Keywords: ergodic point process, Poisson process, harness process, Voronoi tessellation, Delaunay triangulation, Palm measures, ergodic theorem. 


\section{Sumário}

Lista de Símbolos $\quad$ ix

1 Introdução 1

1.1 Considerações Preliminares . . . . . . . . . . . . . . . . . . . 2

1.1.1 Funções Harmônicas em Grafos . . . . . . . . . . . . . . . . . . . . . 2

1.1.2 Ladrilhamento de Voronoi e o Grafo de Delaunay . . . . . . . . . . . . . . . . 3

1.1 .3 A Deformação Harmônica de $\mathcal{G} \ldots \ldots$. . . . . . . . . . . . . . . . 4

1.1.4 O Processo de Harness . . . . . . . . . . . . . . . . . . . . . . . . . 7

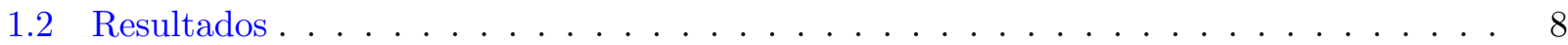

1.3 Organização do Trabalho . . . . . . . . . . . . . . . . . . 9

2 Processos Pontuais $\quad 11$

2.1 O Processo de Poisson . . . . . . . . . . . . . . . . . . . . 12

2.1 .1 A triangulação de Delaunay . . . . . . . . . . . . . . . . . . . . 13

2.2 O Espaço das Medidas de Contagem . . . . . . . . . . . . . . . . . 16

2.3 Processos Pontuais Estacionários . . . . . . . . . . . . . . . . . . . . . . . . 19

2.3.1 Processos Pontuais Ergódicos e Mixing . . . . . . . . . . . . . . . . . . 21

2.4 Medidas de Palm . . . . . . . . . . . . . . . . . . . . . . . 23

2.4.1 Shifts Bijetivos e a Estacionariedade Pontual . . . . . . . . . . . . . . . 29

3 O Passeio Aleatório e o Meio visto da Partícula 33

3.1 Uma breve revisão sobre Processos de Markov . . . . . . . . . . . . . . . . . . . . . . . 34 
3.2 O Passeio Aleatório em $\mathcal{G}$ e o Meio Visto da Partícula . . . . . . . . . . . . 35

4 O Processo de Harness $\quad 41$

4.1 O Processo de Harness sem Ruído . . . . . . . . . . . . . . . . . . . . . . . . . . 41

4.1 .1 A Construção Gráfica . . . . . . . . . . . . . . . . . . . . . . . . . 41

4.2 Funções harmônicas em $\mathcal{G} \ldots \ldots \ldots$. . . . . . . . . . . . . . . . . . . 44

$4.3 \mathrm{O}$ espaço $\mathcal{H} \ldots \ldots \ldots \ldots \ldots \ldots \ldots \ldots \ldots$

$4.4 \mathrm{O}$ processo de Harness como uma sequência em $\mathcal{H} \ldots \ldots$. . . . . . . . . . . . . . 48

4.4 .1 Inclinação . . . . . . . . . . . . . . . . . . . . . 55

4.5 O Resultado Principal . . . . . . . . . . . . . . . . . . . . . . 62

5 Considerações Finais $\quad 71$

5.1 Aplicação - O Princípio de Invariância . . . . . . . . . . . . . . . . . . . . 71

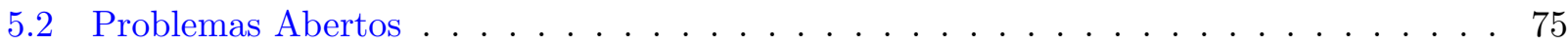

$\begin{array}{ll}\text { A Resultados Auxiliares } & \mathbf{7 7}\end{array}$

A.1 Funções Harmônicas em Grafos Finitos . . . . . . . . . . . . . . . . . . . . 77

A.2 A Medida de Campbell . . . . . . . . . . . . . . . . . . 80

$\begin{array}{lr}\text { Referências Bibliográficas } & 83\end{array}$ 


\section{Lista de Símbolos}

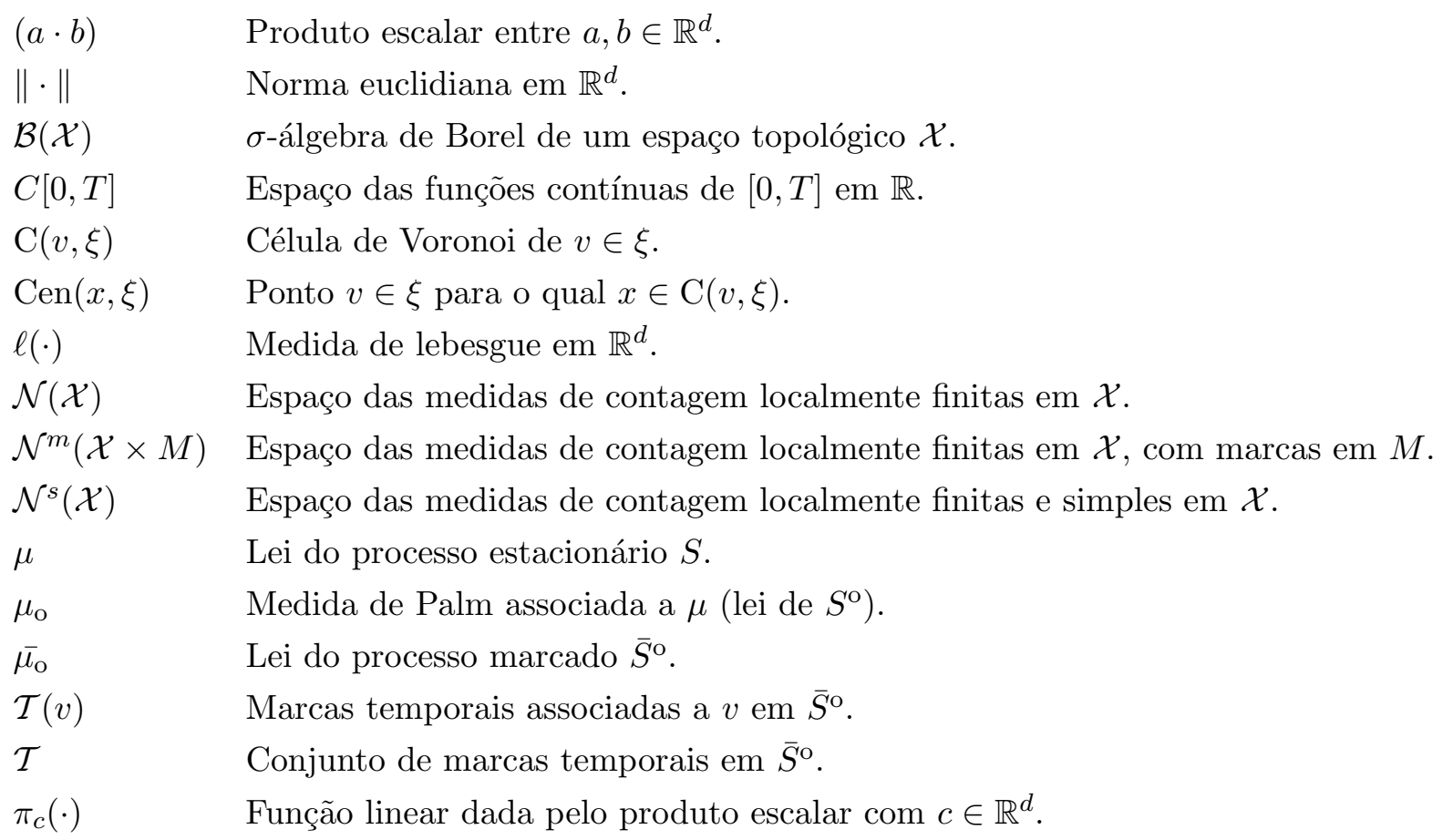




\section{Capítulo 1}

\section{Introdução}

Consideremos o seguinte problema: dado um grafo $\mathcal{G}=(\mathcal{V}, \mathcal{E})$ conexo, imerso em $\mathbb{R}^{d}\left(\mathcal{V} \subset \mathbb{R}^{d}\right)$, é possível encontrar uma nova imersão de $\mathcal{G}$ na qual cada vértice esteja localizado no centro de massa de seu vizinho? Em outras palavras, dado o grafo $\mathcal{G}$, queremos encontrar uma função $h: \mathcal{V} \rightarrow \mathbb{R}^{d}$, não constante, e tal que

$$
h(v)=\frac{1}{|V(v)|} \sum_{w \in V(v)} h(w),
$$

para todo $v \in \mathcal{V}$, onde $V(v)$ é o conjunto dos vértices vizinhos de $v$ em $\mathcal{G}$. De modo similar, podemos pensar no problema coordenada por coordenada, e neste caso o objetivo passa a ser determinar funções $h_{i}: \mathcal{V} \rightarrow \mathbb{R}, i=1, \ldots, d$, preferencialmente distintas, satisfazendo (1.1).

No caso de grafos finitos, a resposta para tal problema é afirmativa (ver Apêndice e [8]), bastando para isso que fixemos a posição de alguns dos vértices (a fronteira). Neste caso, a função determinada satisfaz (1.1) apenas para vértices fora da fronteira.

Para grafos infinitos a questão fica sensivelmente mais complicada, e precisaremos considerar algumas condições e hipóteses adicionais. Suponha primeiro que $\mathcal{G}$ seja tal que, em cada $B \subset \mathbb{R}^{d}$ limitado, existam apenas um quantidade finita de vértices de $\mathcal{V}$. Queremos também que a deformação causada por $h$ não seja "muito grande", o que neste trabalho significará que

$$
\lim _{\|v\| \rightarrow \infty} \frac{\|h(v)-v\|}{\|v\|}=0
$$

Em [4] e [20], os autores estudam este problema para o cluster de percolação independente de elos (caso super-crítico). Em ambos os artigos, a idéia central é definir um corretor que, somado à posição de cada ponto, satisfaça (1.1). No entanto, a verificação da sub-linearidade para o cluster deformado se mostrou um problema ainda mais complicado. Em [4], os autores conseguem determinar a sublinearidade apenas no caso $d=2$. Para outras dimensões os autores verificam apenas versões mais 
fracas de (1.2).

Neste trabalho consideraremos o problema em um grafo aleatório conhecido como Triangulação de Delaunay, construída a partir de pontos de um Processo de Poisson homogêneo em $\mathbb{R}^{d}$. Os argumentos colocados em [4] e [20] poderiam ser facilmente replicados, mas nossa intenção é encontrar uma maneira mais construtiva de resolver o problema. Por esta razão consideraremos aqui uma dinâmica conhecida como processo de Harness, que apresentaremos com mais detalhes um pouco mais a frente. Mas antes, introduziremos nas próximas seções algumas notações e conceitos, deixando mais claro o problema proposto, para depois mostrarmos como pretendemos resolvê-lo.

\subsection{Considerações Preliminares}

\subsubsection{Funções Harmônicas em Grafos}

Dado um conjunto enumerável $\mathcal{V}$ (vértices), associe a cada par $(v, w) \in \mathcal{V} \times \mathcal{V}$ um peso $a(u, v) \geq 0$ com $a(u, v)=a(v, u)$, e tal que $0<a_{v}:=\sum_{w \in \mathcal{V}} a(v, w)<\infty$. Defina $p(v, w)=\frac{a(v, w)}{a_{v}}$. Este conjunto de pesos define um grafo $\mathcal{G}=(\mathcal{V}, \mathcal{E})$ com vértices em $\mathcal{V}$ e conjunto de elos dado por $\mathcal{E}=\{(v, w) \in \mathcal{V} \times \mathcal{V}: a(v, w)>0\}$. Deste ponto em diante consideraremos que o grafo $\mathcal{G}$ assim definido é conexo.

Neste contexto, dado um subconjunto de vértices $\mathcal{V}^{\prime} \subseteq \mathcal{V}$ diremos que uma função $h: \mathcal{V} \rightarrow \mathbb{R}$ é harmônica para $P:=(p(v, w))_{v, w \in \mathcal{V}}$ em $\mathcal{V}^{\prime}$ se

$$
h(v)=\sum_{w \in \mathcal{V}} p(v, w) h(w), \text { para todo } v \in \mathcal{V}^{\prime}
$$

Observe que (1.3) é equivalente a

$$
\sum_{w \in \mathcal{V}} a(v, w)(h(v)-h(w))=0, \text { para todo } v \in \mathcal{V}^{\prime}
$$

No caso em que $\mathcal{V}^{\prime}=\mathcal{V}$ diremos simplesmente que $h$ é harmônica em $\mathcal{G}$. Na teoria de redes elétricas, o peso $a(v, w)$ recebe o nome de condutividade e seu inverso $1 / a(u, v)$ de resistência.

Como não poderia deixar de ser, o estudo de funções harmônicas discretas enfrenta dificuldades sensivelmente diferentes no caso de grafos finitos ou infinitos. Ainda assim, em ambos a relação entre redes elétricas e passeios aleatórios é de substancial importância. É esta ligação que permite, dentre outras coisas, relacionar a resistividade total de uma rede elétrica infinita com a recorrência ou transiência do passeio aleatório associado (ver [8]).

Apesar de considerarmos aqui um grafo infinito, não faremos uso dos resultados existentes para 
este caso. Isso porque a maior parte desta teoria foi desenvolvida para o estudo de funções com energia finita (ver apêndice A), e como ficará claro mais a frente, este não é o nosso caso. Por esta razão não nos estenderemos no assunto. O leitor interessado pode descobrir mais em [25]. No apêndice A faremos um breve resumo de alguns resultados importantes para funções harmônicas em grafos finitos. Trataremos, em especial, do princípio do máximo e do mínimo, que será ferramenta essencial na demonstração de um de nossos resultados.

\subsubsection{Ladrilhamento de Voronoi e o Grafo de Delaunay}

Seja $\xi \subset \mathbb{R}^{d}$ um conjunto enumerável de pontos, tal que $\xi(B):=|\xi \cap B|<\infty(|\cdot|$ denota cardinalidade) para todo $B \subset \mathbb{R}^{d}$ limitado. Dado $v \in \xi$, seja $\mathrm{C}(v):=\mathrm{C}(v, \xi)$ a região aberta de $\mathbb{R}^{d}$ formada por todos os pontos em $\mathbb{R}^{d}$ mais próximos de $v$ que de qualquer outro $w \in \xi$, ou seja,

$$
\mathrm{C}(v)=\left\{x \in \mathbb{R}^{d}:\|x-v\|<\|x-w\| \text {, para todo } w \in \xi, w \neq v\right\} .
$$

O conjunto $\mathrm{C}(v, \xi)$ é conhecido por célula de Voronoi de $v$ associada a $\xi$.

Deste modo, a menos de um subconjunto de medida de Lebesgue 0 , conseguimos dividir $\mathbb{R}^{d}$ em abertos disjuntos $\{\mathrm{C}(v, \xi) ; v \in \xi\}$, formando assim um ladrilhamento de $\mathbb{R}^{d}$ conhecido como ladrilhamento de Voronoi (do inglês 'Voronoi Tessellation'). Note que o fecho de cada célula deste ladrilhamento é um polígono convexo, e que qualquer par destes "ladrilhos" compartilham no máximo uma face. Considere agora o grafo $\mathcal{G}(\xi)=(\xi, \mathcal{E}(\xi))$ formado ao colocarmos um elo entre quaisquer par de sítios $v, w \in \xi$ tais que as respectivas células de Voronoi compartilhem uma face $d-1$ dimensional. O grafo $\mathcal{G}(\xi)$ assim definido é conhecido por grafo de Delaunay ou triangulação de Delaunay associada a $\xi$.

Observação 1.1.1. Nos preocuparemos apenas com configurações de pontos $\xi \subset \mathbb{R}^{d}$ tais que nenhum subconjunto de $d+2$ pontos em $\xi$ está na fronteira de uma mesma bola d dimensional. Nestes casos, outra forma de construir $\mathcal{G}(\xi)$ em $\mathbb{R}^{d}$ é colocar elos entre quaisquer $d+1$ pontos de $\xi$ tais que a bola definida por tais pontos não possua nenhum outro ponto de $\xi$ em seu interior. Esta construção tem a vantagem de possibilitar o cálculo de algumas estimativas, como por exemplo, para o tamanho de um elo. De fato, para um certo elo ser muito grande, deve haver uma região também grande sem nenhum ponto em seu interior.

Neste trabalho consideraremos o grafo de Delaunay associado a um processo de Poisson homogêneo em $\mathbb{R}^{d}$. Este modelo, assim como o ladrilhamento de Voronoi associado, vem sendo amplamente estudado nas últimas décadas. Resultados sobre distribuições e momentos de quantidades relacionadas a tais modelos, como grau de vértices, tamanho de arestas, volume de células, dentre 


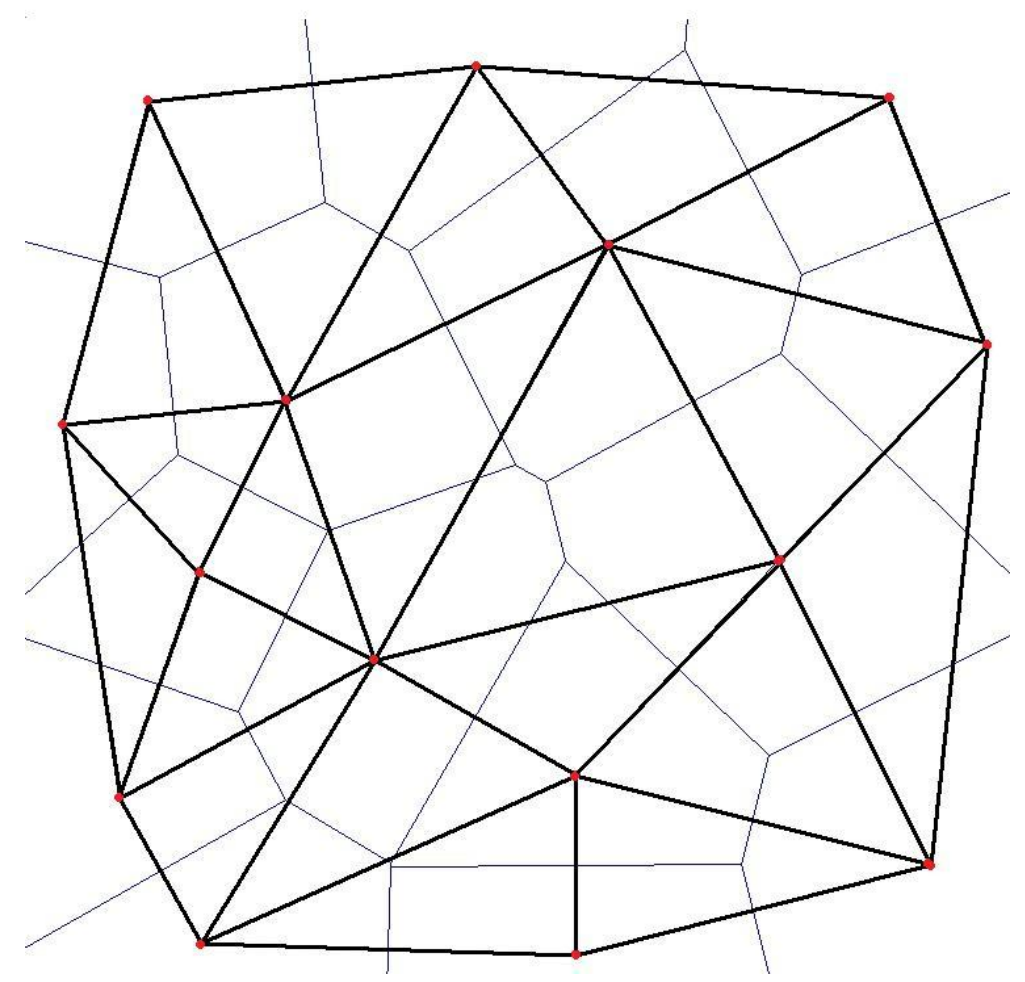

Figura 1.1: Ladrilhamento de Voronoi e a Triangulação de Delaunay associada.

outros podem ser encontrados em [5], [3], [21], [23], [24], e referências ali citadas. As aplicações de tais modelos em outras áreas do conhecimento é também bastante ampla. Para uma aplicação do Ladrilhamento de Voronoi em redes de telecomunicação, ver [1].

Para fixar notação, ao longo deste trabalho usaremos $V(v):=V(v, \xi)$ para notar o conjunto de vizinhos de $v$ em $G(\xi)$, e dado $x \in \mathbb{R}^{d}$, Cen $(x, \xi):=\operatorname{Cen}(x)$ representará o ponto $v \in \xi$ tal que $x \in \mathrm{C}(v)$.

\subsubsection{A Deformação Harmônica de $\mathcal{G}$}

Seja $S$ um processo de Poisson homogêneo em $\mathbb{R}^{d}$, e $\mathcal{G}(S)=(S, \mathcal{E}(S))$ o grafo de Delaunay associado à $S$. A cada par de pontos $v, w \in S$ associe um peso $a(v, w)=a(v, w, S)$ dado por

$$
a(v, w, S)=\mathbf{1}\{(v, w) \in \mathcal{E}(S)\}
$$

Defina então $a(v, S):=|V(v)|=\sum_{w \in S} a(v, w)$. 
Considere a matriz $P(S):=(p(v, w, S))_{v, w}$ dada por

$$
p(v, w, S):=\frac{a(v, w, S)}{a(v, S)} .
$$

Lembrando, nosso objetivo é, dada um configuração de $S$, encontrar funções $h_{i}: S \rightarrow \mathbb{R}, i=$ $1, \ldots d$, harmônicas para $P$ em $S$, e tais que $v \in \mathcal{V} \mapsto h(v):=\left(h_{1}(v), \ldots, h_{d}(v)\right)$ satisfaça $(1.2)$.

Simplificando, estamos interessados na caracterização de funções harmônicas para $P$ em $S$, ou seja, funções harmônicas em $\mathcal{G}$. Em particular, olhando para $\mathcal{G}$ como um grafo naturalmente imerso em $\mathbb{R}^{d}$, queremos encontrar funções harmônicas com comportamento assintótico linear.

Para deixar mais claro nossos objetivos, dado $c=\left(c_{1}, \ldots, c_{d}\right) \in \mathbb{R}^{d}$, denote por $\pi_{c}$ a função linear (ou hiper-plano) $x \mapsto \pi_{c}(x):=(c \cdot x)=c_{1} x_{1}+\cdots+c_{d} x_{d}$. Nossa intenção é verificar se existe uma função $h_{c}$, harmônica em $G$ e com mesmo comportamento assintótico linear de $\pi_{c}$, ou seja,

$$
\lim _{\|v\| \rightarrow \infty} \frac{\left|h_{c}(v)-\pi_{c}(v)\right|}{\|v\|}=0
$$

Infelizmente, só seremos capazes de resolver este problema em $d=2$. Neste caso mostraremos, utilizando as mesmas técnicas descritas em [4], que

$$
\lim _{n \rightarrow \infty} \max _{v \in S \cap[-n, n]^{2}} \frac{\left|h_{c}(v)-\pi_{c}(v)\right|}{n}=0, \quad \text { q.c. }
$$

Para o caso geral, verificaremos a sub-linearidade de $h_{c}-\pi_{c}$ apenas em direções fixas. Para que possamos esclarecer nossas intenções, é necessário que definamos o que entenderemos por inclinação de uma dada função em uma direção fixa.

Para acomodar uma possível dependência em $S$ das funções consideradas defina

$$
\Xi_{1}=\left\{(v, \xi) \in \mathbb{R}^{d} \times \mathcal{N}: v \in \xi\right\}
$$

onde $\mathcal{N}$ denota o espaço onde vivem os processos pontuais em $\mathbb{R}^{d}$, a ser definido no capítulo 2 . Posteriormente, será necessário ampliar a definição do espaço $\Xi_{1}$ para acomodar também processos pontuais marcados, mas por hora esta definição nos basta.

Na definição abaixo, $\mu$ denotará a lei do processo pontual considerado.

Definição 1.1. Dada uma direção $u \in \mathbb{R}^{d},\|u\|=1$, diremos que $f: \Xi_{1} \rightarrow \mathbb{R}$ tem inclinação $I_{u}(f)$ na direção $u$ dada por

$$
I_{u}(f):=\lim _{t \rightarrow \infty} \frac{|f(\operatorname{Cen}(v+t u, \xi), \xi)-f(v, \xi)|}{t}
$$



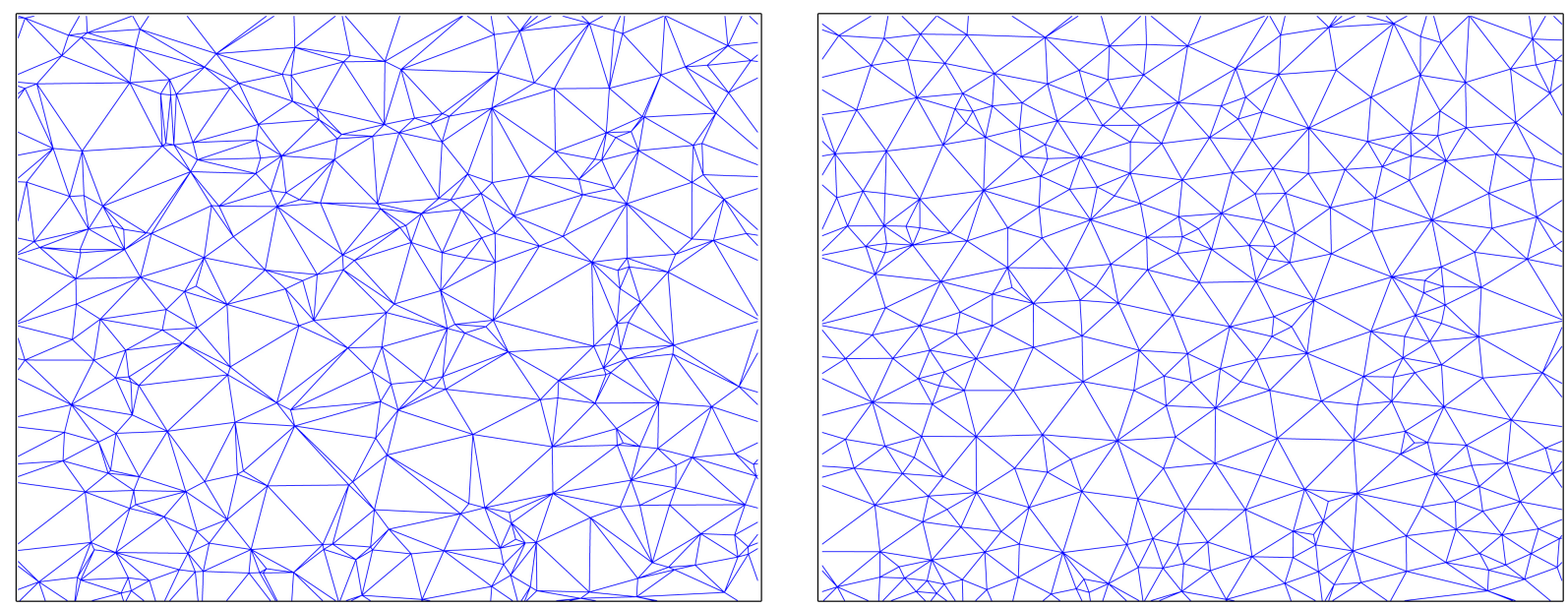

Figura 1.2: Triangulação de Delaunay associada a um processo de Poisson, e a respectiva deformação harmônica.

sempre que o limite acima existe $\mu$-quase certamente e não depende de $v \in \xi$.

Note que $I_{u}(\cdot)$ é linear e, portanto, dizer que $I_{u}(h)=I_{u}(\pi)$ é equivalente a dizer que $I_{u}(\pi-h)=0$, ou seja, $\pi-h$ é sub-linear na direção $u$.

Voltando agora para o problema inicial, considere os planos coordenados $\pi_{i}(x)=x_{i}, i=1, \ldots, d$, e suponha que para cada $i$ seja possível encontrar funções harmônicas $h_{i}$ satisfazendo (1.8). Tome agora $h: S \rightarrow \mathbb{R}^{d}$ dada por $v \in \mathcal{V} \mapsto h(v):=\left(h_{1}(v), \cdots, h_{d}(v)\right)$, e defina uma nova imersão de $\mathcal{G}$ em $\mathbb{R}^{d}$ reposicionando cada ponto $v \in S$ para uma nova posição dada por $h(v)$, e mantendo os elos originais. Mais precisamente, defina $\mathcal{G}^{\prime}(S)=\left(S^{\prime}, \mathcal{E}^{\prime}(S)\right)$ dado por

$$
S^{\prime}=\{h(v) ; v \in S\}
$$

e

$$
\mathcal{E}^{\prime}(S)=\{(h(v), h(w)) ;(v, w) \in \mathcal{E}(S)\}
$$

O grafo $\mathcal{G}^{\prime}$ tem portanto a propriedade de cada ponto $v^{\prime} \in S^{\prime}$ estar posicionado no centro de massa de seus vizinhos, ou seja,

$$
\sum_{\substack{w^{\prime} \in S^{\prime}: \\\left(v^{\prime}, w^{\prime}\right) \in \mathcal{E}^{\prime}}}\left(v^{\prime}-w^{\prime}\right)=0,
$$

para todo $v^{\prime} \in S^{\prime}$. Chamaremos $\mathcal{G}^{\prime}$ de deformação harmônica de $\mathcal{G}(S)$. Em [4] e [20] os autores estudam tal deformação para o cluster de percolação supercrítico. Em ambos, o estudo se faz através da definição de um corretor, mas de maneiras ligeiramente distintas (em [20] são utilizados argumentos 
projetivos, e em [4] os autores usam análise espectral). Em ambos os artigos os autores falham em mostrar que o corretor definido satisfaz (1.8). Única exceção feita a [4] em que, para o caso $d=2$, os autores mostram que vale (1.9).

\subsubsection{O Processo de Harness}

No sentido de tentar buscar uma resposta para os problemas colocados acima, vamos utilizar uma dinâmica conhecida por Processo de Harness. O conceito de Harnesses foi introduzido por Hammersley em [14]. Harnesses são medidas em $\mathbb{R}^{\mathbb{Z}^{d}}$ com a propriedade de que o valor médio da altura em cada sítio $z \in \mathbb{Z}^{d}$ é a média (pesada por uma matriz de transição de Markov $(P(i, j))_{i, j \in \mathbb{Z}^{d}}$, com $P(i, i)=0)$ do valor médio das alturas em todos os sítios diferentes de $z$. É interessante observar que, o uso de $\mathbb{Z}^{d}$ nesta definição é apenas para fixar idéias, não impedindo em nada sua extensão para qualquer conjunto discreto de pontos.

Tal conceito foi introduzido com o propósito de modelar o comportamento de um cristal e também introduzir uma versão multi-dimensional de martigal. O processo conhecido por serial Harness, é um processo Markoviano a tempo discreto, com valores em $\mathbb{R}^{\mathbb{Z}^{d}}$ onde, a cada instante $t>0$, a altura em cada ponto é atualizada para a altura média pesada por $P$ da altura dos outros pontos em $\mathbb{Z}^{d}$ no tempo $t-1$, mais uma variável centrada independente, denominada de ruído. O modelo a tempo contínuo, conhecido por processo de Harness, foi introduzido por Hsiao em [17] e consiste em atualizar cada ponto em tempos de Poisson independentes, seguindo a mesma regra do modelo a tempo discreto.

Em [11], Ferrari et. al. mostram que se o ruído é dado por variáveis Gaussianas centradas, então a medida de Gibbs associada com o Hamiltoniado quadrático

$$
H(\eta)=\sum_{i, j} P(i, j)(\eta(i)-\eta(j))^{2}
$$

onde $\eta \in \mathbb{R}^{\mathbb{Z}^{d}}$, e temperatura $1 / \beta$ dada pela variância do ruído, é reversível para o processo de Harness.

Em [12], Ferrari et. al. estudam uma versão do processo de harness com influência de campo externo fixo $d \in \mathbb{R}^{\mathbb{Z}^{d}}$. Neste modelo, cada sítio $i \in \mathbb{Z}^{d}$ tem um vizinho externo $i^{*}$ com altura fixa $d(i)$, e a atualização é feita considerando peso $\alpha$ para o vizinho no sítio externo e peso $(1-\alpha) P(i, j)$ para $j \in \mathbb{Z}^{d}$. Entre outros resultados, os autores mostram que o processo sem ruído converge para uma única função harmônica $m$ (com atuação do campo externo). Mais precisamente, o processo 
sem ruído converge para uma configuração $m \in \mathbb{R}^{\mathbb{Z}^{d}}$ tal que

$$
m(i)=(1-\alpha) \sum_{j \in \mathbb{Z}^{d}} P(i, j) m(j)+\alpha d(i)
$$

para todo $i \in \mathbb{Z}^{d}$

Neste trabalho consideraremos uma versão "multi-dimensional" de um processo de Harness sem ruído na triangulação $\mathcal{G}(S)$. Em palavras, fixada uma realização de $S$, a posição de cada ponto será atualizada em tempos de Poisson de taxa 1 para o centro de massa dos seus vizinhos.

De maneira equivalente, seguindo mais de perto a idéia do processo de Harness, podemos considerar que os pontos de $S$ estão fixos, e cada um deles associamos um vetor de alturas, evoluindo no tempo de acordo com as regras de um processo Harness. Em outras palavras, teriamos um processo $\left(\eta_{t}^{i}, i=1, \ldots, d\right)_{t \geq 0}$, onde para cada $i=1, \ldots, d, \eta_{t}^{i}$ é um processo de Harness sem ruído definido nos pontos de um processo pontual $S$, utilizando pesos $P(S)=(p(v, w))_{v, w \in S}$ definidos em (1.7).

De modo um pouco mais geral, fixada uma realização de $S$, construiremos o processo de Harness $\eta_{t}^{\gamma}$ em $\mathcal{G}(S)$, com $\eta_{0}^{\gamma} \equiv \gamma$, como função de um processo $S$ em $\mathbb{R}^{d}$ e de marcas temporais associadas a cada $v \in S$. Mostraremos que, começando com uma configuração linear $\pi \in \mathbb{R}^{S}$ o processo de Harness sem ruído em $\mathcal{G}(S)$ converge (de maneira que ficará clara mais a frente) para uma função harmônica $h$ em $\mathcal{G}(S)$, com o mesmo comportamento assintótico de $\pi$ em qualquer direção coordenada. Mais precisamente, dada uma direção $u \in \mathbb{R}^{d}$, mostraremos que $I_{u}(h)=I_{u}(\pi)$. Adaptando as técnicas descritas em [4] mostraremos que no caso $d=2$ vale (1.9).

\subsection{Resultados}

Para $i=1 \ldots, d$, denote por $\pi_{i}$ o $i$-ésimo hiper-plano coordenado, ou seja, $\pi_{i}(x)=x_{i}$, e considere $\eta_{t}^{i}$ o processo de Harness sem ruído em $\mathcal{G}(S)$, com condição inicial dada por $\eta_{0}^{i} \equiv \pi_{i}$. Para $\phi: \Xi_{1} \rightarrow \mathbb{R}$, faça $\nabla \phi(w, v, S)=\phi(v, S)-\phi(w, S)$.

Teorema 1.2. Seja $S$ é um Processo de Poisson homogêneo em $\mathbb{R}^{d}$, e $S^{\mathrm{o}}=S \cup\{0\}$. Então, para quase toda realização de $S^{\circ}$, e para cada $i=1, \ldots, d$, existe $h_{i}: \Xi_{1} \rightarrow \mathbb{R}$, harmônica em $\mathcal{G}\left(S^{\circ}\right)$, com $I_{e}\left(\pi_{i}-h_{i}\right)=0$ para toda direção coordenada $e \in \mathbb{R}^{d}$, e tal que

$$
\lim _{t \rightarrow \infty} \mathbb{E}\left[a(0, v)\left|\nabla \eta_{t}^{i}(0, v)-\nabla h_{i}(0, v)\right|^{2}\right]=0
$$

para todo $v \in S^{\mathrm{o}}$.

Para $d=2$ vale o seguinte resultado. 
Teorema 1.3. Se $h: \Xi_{1} \rightarrow \mathbb{R}$ é uma função harmônica em $\mathcal{G}\left(S^{\circ}\right)$ com $I_{e_{1}}(h-\pi)=I_{e_{2}}(h-\pi)=0$ para alguma função linear $\pi: \mathbb{R}^{2} \rightarrow \mathbb{R}$, então

$$
\lim _{n \rightarrow \infty} \max _{v \in S^{\mathrm{O}} \cap[-n, n]^{2}}\left\{\frac{\left|h\left(v, S^{\mathrm{o}}\right)-\pi(v)\right|}{n}\right\}=0, \quad \text { q.c.. }
$$

Observação 1.2.1. Apesar do resultado estar enunciado para um processo de Poisson, as técnicas utilizadas em sua demonstração permitem estabelecer o mesmo resultado para outros processos pontuais estacionários, desde que algumas condições sobre a distribuição do processo sejam verificadas. No início do Capítulo 3 listaremos as hipóteses a serem cumpridas pelo processo pontual considerado, e comentaremos brevemente cada uma delas.

Como consequência dos dois teoremas acima, no final do trabalho, vamos determinar o princípio de invariância para o passeio aleatório em $\mathcal{G}$. Esta aplicação é, na verdade, a razão principal que levaram os autores em [4] e [20], a estudarem a deformação harmônica do cluster de percolação. A idéia, introduzida por Kipnis e Varadhan em [18], é primeiro estudar o passeio no grafo deformado, onde este é um martingal, e encontrar o princípio de invariância para este novo processo. Depois, usando a sub-linearidade da deformação, mostrar que a diferença entre os dois passeios desaparece após reescalada.

\subsection{Organização do Trabalho}

No Capítulo 2, traremos um pequeno resumo dos principais conceitos e resultados relacionados a processos pontuais necessários para o nosso resultado. Começaremos apresentando uma construção do Processo de Poisson homogêneo, e faremos algumas considerações sobre o grafo de Delaunay relacionado. Passaremos então para o estudo de processos pontuais em geral. Trataremos em particular de processos estacionários, onde abordaremos resultados relacionados as medidas de Palm, incluindo o Teorema Ergódico Espacial.

No Capítulo 3 faremos um breve estudo do passeio aleatório no grafo $\mathcal{G}$, trazendo resultados essenciais para a sequência de nosso trabalho.

O Capítulo 4 trata dos problemas propostos neste trabalho. Nele faremos a construção gráfica do processo de Harness usando passeios aleatórios. Introduziremos o espaço de Hilbert onde vive nosso processo, tentando caracterizar alguns dos elementos deste espaço. Para concluir faremos a demonstração dos resultados propostos.

Para finalizar, no capítulo 5 , vamos demonstrar o princípio de invariância para o passeio aleatório em $\mathcal{G}$ no caso $d=2$. Encerramos então fazendo um breve resumo dos problemas ainda não solucionados neste trabalho. 
O apêndice trará um resumo de alguns resultados sobre funções harmônicas discretas. Trará também uma breve justificativa de alguns passos técnicos utilizados ao longo do texto. 


\section{Capítulo 2}

\section{Processos Pontuais}

Neste capítulo vamos estudar uma classe de processos estocásticos espaciais conhecidos como Processos Pontuais. Nas próximas seções vamos definir o que entenderemos por Processo Pontual, resumindo os principais resultados relacionados a tais modelos. Daremos especial atenção à família de processos estacionários, explorando suas principais características e propriedades. Através das medidas de Palm, estudaremos o comportamento de tais modelos na vizinhança de um "ponto típico". Finalmente, no final do capítulo, estudaremos brevemente os shifts-bijetivos e as relações com a dualidade "estacionariedade/estacionariedade pontual".

Como já salientado na introdução deste trabalho, apesar de boa parte (senão a totalidade) dos resultados apresentados neste trabalho serem válidos para processos pontuais satisfazendo determinadas condições, nosso principal interesse está nos processos de Poisson que, apenas pela posição de destaque que ocupam na teoria de processos pontuais, já mereceriam um capítulo a parte. Por esta razão, antes de introduzir formalmente a noção de Processo Pontual, vamos apresentar uma breve construção de tais processos.

Antes de começar vamos fixar algumas das notações que utilizaremos ao longo dos próximos capítulos. Deste ponto em diante, fixado um espaço métrico $\mathcal{X}$, denotaremos por $\mathcal{B}(\mathcal{X})$ a $\sigma$-álgebra dos borelianos de $\mathcal{X}$. Utilizaremos $\ell(\cdot)$ para denotar a medida de lebesgue em $\left(\mathbb{R}^{d}, \mathcal{B}\left(\mathbb{R}^{d}\right)\right)$ e, correndo de risco de causar alguma ambiguidade, utilizaremos $|\cdot|$ para denotar tanto a cardinalidade de um conjunto, como o valor absoluto de um número real. Usaremos 0 para denotar a origem em $\mathbb{R}^{d} \mathrm{e}$, finalmente, denotaremos por $\|\cdot\|$ a norma euclidiana e por $(a \cdot b)$ o produto escalar convencional em $\mathbb{R}^{d}$, ou seja, $\|x\|^{2}=(x \cdot x)$. 


\subsection{O Processo de Poisson}

Seja $\rho: \mathbb{R}^{d} \rightarrow \mathbb{R}$ positiva e localmente integrável, ou seja,

$$
\mu(B):=\int_{B} \rho(x) d x<\infty
$$

para todo $B \in \mathcal{B}\left(\mathbb{R}^{d}\right)$ limitado. Considere agora uma partição $P:=\left\{A_{n} \in \mathcal{B}\left(\mathbb{R}^{d}\right) ; n \geq 0\right\}$ de $\mathbb{R}^{d}$ formada apenas por borelianos limitados. Apenas para fixar idéias, podemos pensar, por exemplo, em uma partição formada por retângulos. Seja $\left\{N_{n} ; n \geq 0\right\}$ uma coleção de variáveis aleatórias independentes com distribuição Poisson de parâmetro $\mu\left(A_{n}\right)$. Em seguida, para cada $n \geq 0$, sorteie $N_{n}$ pontos e distribua-os independentemente em $A_{n}$ de acordo com uma distribuição de densidade $\left(\rho \cdot \mathbf{1}_{A_{n}}\right)(\cdot) / \mu\left(A_{n}\right)$, denominando este conjunto aleatório de pontos de $X_{n}$. Defina agora

$$
S:=\bigcup_{n \geq 0} X_{n}
$$

É fácil verificar (ver [22]) que a construção apresentada acima não depende da escolha da partição $P$ e, além disso, o processo $S$ possui as seguintes propriedades:

(i) Para cada $B \in \mathcal{B}\left(\mathbb{R}^{d}\right)$ limitado, $S(B):=|S \cap B|$ tem lei Poisson de parâmetro $\mu(B)$;

(ii) $S(B)$ e $S(A)$ são independentes sempre que $A \cap B=\emptyset$;

O Processo $S$ assim definido é conhecido como processo de Poisson em $\mathbb{R}^{d}$, e $\rho$ é chamado de função intensidade de $S$. No caso em que $\rho(\cdot) \equiv \lambda<\infty$ é constante, diremos que o processo de Poisson é homogêneo.

A seguir enunciaremos, sem provar, um importante resultado para processos de Poisson: a Fórmula de Slivnyak-Mecke. O leitor interessado em uma prova pode ver, por exemplo, [22].

Proposição 2.1 (Fórmula de Slivnyak-Mecke). Se $S$ é um processo de Poisson em $\mathbb{R}^{d}$ com função densidade $\rho$, e $f:\left(\mathbb{R}^{d}\right)^{n} \times \mathcal{N} \rightarrow \mathbb{R}$ é positiva e mensurável, então

$$
\mathbb{E}\left[\sum_{x_{1}, \ldots, x_{n} \in S} f\left(x_{1}, \ldots, x_{n}, S\right)\right]=\int_{\mathbb{R}^{d}} \ldots \int_{\mathbb{R}^{d}} \mathbb{E}\left[f\left(x_{1}, \ldots, x_{n}, S \cup\left\{x_{1}, \ldots, x_{n}\right\}\right)\right] \prod_{k=1}^{n} \rho\left(x_{k}\right) d x_{k} .
$$

Na proposição acima, $\mathcal{N}$ representa o espaço onde vivem os processos pontuais, e será definido e estudado na próxima seção. 


\subsubsection{A triangulação de Delaunay}

Vamos agora nos concentrar em conseguir algumas estimativas, necessárias em resultados futuros, para algumas características do grafo de Delaunay formado a partir de um processo de Poisson homogêneo de intensidade $\rho$. Em particular, estamos interessados em estudar a estrutura da vizinhança de um "ponto típico" do processo. Como será justificado mais a frente, uma maneira de fazer isso para um processo de Poisson homogêneo $S$, é considerar o processo $S^{\circ}:=S \cup\{0\}$, e estudar o que acontece em torno do ponto na origem.

Comecemos com o volume da célula de Voronoi da origem em $S^{\mathrm{o}}$. Observe então que para $x \in \mathbb{R}^{d}$ esteja na célula de Voronoi de 0 , precisamos que bola aberta de centro em $x$ e raio $\|x\|$ não possua nenhum ponto de $S^{\mathrm{o}}$, ou seja,

$$
\mathbb{P}\left(x \in \mathrm{C}\left(0, S^{\mathrm{o}}\right)\right)=\mathbb{P}(S \cap B(x,\|x\|)=\emptyset)=e^{-\rho \ell(B(x,\|x\|))}=e^{-\rho \omega_{d}\|x\|^{d}},
$$

onde $\omega_{d}$ é o volume da bola unitária em $\mathbb{R}^{d}$. Do mesmo modo, para $x, y \in \mathbb{R}^{d}$

$$
\mathbb{P}\left(x, y \in \mathrm{C}\left(0, S^{\mathrm{o}}\right)\right)=\mathbb{P}(S \cap\{B(x,\|x\|) \cup B(y,\|y\|)\}=\emptyset)=e^{-\rho \ell(B(x,\|x\|) \cup B(y,\|y\|))} \leq e^{\frac{-\rho \omega_{d}}{2}\left(\|x\|^{d}+\|y\|^{d}\right)} .
$$

Deste modo

$$
\mathbb{E}\left[\ell\left(\mathrm{C}\left(0, S^{\mathrm{o}}\right)\right]=\mathbb{E}\left[\int \mathbf{1}_{\mathrm{C}(0)}(x) d x\right]=\int e^{-\rho \omega_{d}\|x\|^{d}} d x=\frac{1}{\rho} .\right.
$$

Em realidade, pode-se mostrar que o resultado acima é valido para qualquer processo pontual estacionário de intensidade finita (ver [27]). Vale também que

$$
\mathbb{E}\left[\mid \ell\left(\left.\mathrm{C}\left(0, S^{\mathrm{o}}\right)\right|^{2}\right]=\mathbb{E}\left[\left(\int \mathbf{1}_{\mathrm{C}(0)}(x) d x\right)\left(\int \mathbf{1}_{\mathrm{C}(0)}(y) d y\right)\right] \leq \iint e^{\frac{-\rho \omega_{d}}{2}\left(\|x\|^{d}+\|y\|^{d}\right)} d x d y<\infty .\right.
$$

E procedendo da mesma maneira, mostra-se que

$$
\mathbb{E}\left[\mid \ell\left(\left.\mathrm{C}\left(0, S^{\mathrm{o}}\right)\right|^{k}\right]<\infty\right.
$$

para todo $k \geq 0$.

Dado agora um ponto $v \in S^{\mathrm{o}}$, vamos tentar estimar (mesmo que grosseiramente) a probabilidade deste ponto ser vizinho da origem. Para isso lembre que, pela Observação 1.1.1, $v$ é vizinho da origem se, e somente se, existirem $v_{1}, \ldots, v_{d-1} \in S^{\text {o }}$ tais que a bola aberta $B^{0, v, v_{1}, \ldots, v_{d-1}}$ determinada por $0, v, v_{1}, \ldots, v_{d-1}$ não possui nenhum ponto de $S^{\circ}$ em seu interior. Neste sentido, fixado $x \in \mathbb{R}^{d}$, 
considere então o evento

$$
A_{x}=\left\{\exists v_{1}, \ldots, v_{d-1} \in S^{\mathrm{o}} ; B^{x, v_{1}, \ldots, v_{d-1}} \cap S^{\mathrm{o}}=\emptyset\right\}
$$

e observe que o evento $A_{x}$ depende apenas da configuração de pontos no interior de bolas que possuam o ponto $x$ e a origem na fronteira, de modo que $\left\{S^{\circ} \in A_{x}\right\}=\left\{S \backslash\{x\} \in A_{x}\right\}$.

Note agora que para $x \in \mathbb{R}^{d}$, e pela Proposição 2.1 segue que

$$
\begin{aligned}
\mathbb{P}\left(S \in A_{x}\right) & \leq \mathbb{E}\left[\sum_{v_{1}, \ldots, v_{d-1} \in S} \mathbf{1}_{\left\{B^{\left.0, x, v_{1}, \ldots, v_{d-1} \cap S=\emptyset\right\}}\right]}\right] \\
& =\int_{\mathbb{R}^{d}} \cdots \int_{\mathbb{R}^{d}} \mathbb{P}\left(B^{0, x, x_{1}, \ldots, x_{d-1}} \cap S=\emptyset\right) d x_{1} \cdots d x_{d-1} \\
& =\int_{\mathbb{R}^{d}} \cdots \int_{\mathbb{R}^{d}} e^{-\ell\left(B^{\left.0, x, x_{1}, \ldots, x_{d-1}\right)} d x_{1} \cdots d x_{d-1} .\right.}
\end{aligned}
$$

Mas o raio $r$ de $B^{x, x_{1}, \ldots, x_{d-1}}$ é tal que

$$
2 r \geq\|x\|, \text { e } r \geq\left\|x_{i}\right\|, \quad i=1, \ldots, d-1 .
$$

Portanto

$$
r \geq \frac{1}{2 d}\left(\|x\|+\left\|x_{1}\right\| \cdots+\left\|x_{d-1}\right\|\right)
$$

$\mathrm{e}$

$$
\mathbb{P}\left(S \in A_{x}\right) \leq \int_{\mathbb{R}^{d}} \cdots \int_{\mathbb{R}^{d}} e^{-K_{d}\left(\|x\|^{d}+\left\|x_{1}\right\|^{d} \cdots+\left\|x_{d-1}\right\|^{d}\right)} d x_{1} \cdots d x_{d-1}=C_{d} e^{-K_{d}\|x\|^{d}},
$$

onde $C_{d}=\left(\int e^{-K_{d}\|x\|^{d}} d x\right)^{d-1}, K_{d}=w_{d}(2 d)^{-d}$. 
Vamos agora estimar $\mathbb{P}\left(S \in A_{x} \cap A_{y}\right)$. Para isso, note que

$$
\begin{aligned}
\mathbb{P}\left(S \in A_{x} \cap A_{y}\right) & \leq \mathbb{E}\left[\sum_{v_{1}, \ldots, v_{d-1} \in S} \sum_{w_{1}, \ldots, w_{d-1} \in S} \mathbf{1}_{\left\{B^{\left.0, x, v_{1}, \ldots, v_{d-1} \cap S=\emptyset\right\}}\right.} \mathbf{1}_{\left\{B^{\left.0, y, w_{1}, \ldots, w_{d-1} \cap S=\emptyset\right\}}\right.}\right] \\
& \leq \int_{\mathbb{R}^{d}} \cdots \int_{\mathbb{R}^{d}} \mathbb{P}\left(\left\{\left(B^{0, x, x_{1}, \ldots, x_{d-1}} \cup B^{0, y, y_{1}, \ldots, y_{d-1}}\right) \cap S=\emptyset\right) \prod_{k=1}^{d-1} d x_{k} d y_{k}\right. \\
& =\int_{\mathbb{R}^{d}} \cdots \int_{\mathbb{R}^{d}} e^{-\ell\left(B^{0, x, x_{1}, \ldots, x_{d-1}} \cup B^{0, y, y_{1}, \ldots, y_{d-1}}\right)} \prod_{k=1}^{d-1} d x_{k} d y_{k} \\
& =\int_{\mathbb{R}^{d}} \cdots \int_{\mathbb{R}^{d}} e^{-\frac{1}{2} \ell\left(B^{\left.0, x, x_{1}, \ldots, x_{d-1}\right)} e^{-\frac{1}{2} \ell\left(B^{0, y, y_{1}, \ldots, y_{d-1}}\right)} \prod_{k=1}^{d-1} d x_{k} d y_{k}\right.} \\
& \leq C_{d}^{(2)} e^{-\frac{K_{d}}{2}\left(\|x\|^{d}+\|y\|^{d}\right)},
\end{aligned}
$$

onde $C_{d}^{(2)}=\left[\int e^{-\frac{K_{d}}{2}\|x\|^{d}} d x\right]^{2(d-1)}$.

Utilizando os mesmos argumentos, podemos mostrar então que

$$
\mathbb{P}\left(S \in \cap_{k=1}^{n} A_{x_{k}}\right) \leq C_{d}^{(n)} \prod_{k=1}^{n} e^{-\frac{K_{d}}{n}\left\|x_{k}\right\|^{d}},
$$

$\operatorname{com} C_{d}^{(n)}=\left[\int e^{-\frac{K_{d}}{n}\|x\|^{d}} d x\right]^{n(d-1)}$.

Deste modo, utilizando mais uma vez a Proposição 2.1, podemos concluir que, para todo $n \geq 0$ e $c \in \mathbb{R}^{d}$

$$
\begin{array}{r}
\mathbb{E}\left[\sum_{v \in S^{\circ}} a\left(0, v, S^{\mathrm{o}}\right)|(c \cdot v)|^{n}\right]=\mathbb{E}\left[\sum_{v \in S}|(c \cdot v)|^{n} \mathbf{1}_{A_{v}}(S)\right]=\int_{\mathbb{R}^{d}}|(c \cdot x)|^{n} C_{d} e^{-K_{d}\|x\|^{d}} d x<\infty . \\
\mathbb{E}\left[|V(0)|^{n}\right]=\mathbb{E}\left[\sum_{v_{1}, \cdots, v_{n}} \mathbf{1}_{\cap_{k=1}^{n} A_{v_{k}}}(S)\right]=\int_{\mathbb{R}^{d}} \cdots \int_{\mathbb{R}^{d}} \mathbb{P}\left(S \in \cap_{k=1}^{n} A_{x_{k}}\right) d_{x_{1}} \cdots d_{x_{n}}<\infty
\end{array}
$$

Outro conjunto que precisaremos estudar é

$$
V_{2}=\bigcup_{v \in V(0)} V(v)
$$

ou seja, todos os pontos com distância para a origem menor ou igual a 2 (na métrica natural de $\mathcal{G}$ ). 
Para estimarmos os momentos de $\left|V_{2}\right|$, considere os eventos

$$
A_{x, y}=\left\{\exists v_{1}, \ldots, v_{d-1} \in S^{\mathrm{o}} ; B^{x, y, v_{1}, \ldots, v_{d-1}} \cap S^{\mathrm{o}}=\emptyset\right\}
$$

$\mathrm{e}$

$$
A_{y}^{2}=\left\{\exists v \in S^{\mathrm{o}}: S^{\mathrm{o}} \in A_{v} \cap A_{v, y}\right\} .
$$

Usando os mesmos argumentos utilizados acima, podemos mostrar que

$$
\begin{gathered}
\mathbb{P}\left(S \in A_{x, y}\right) \leq C_{d} e^{-K_{d}\|x-y\|^{d}}, \\
\mathbb{P}\left(S \in A_{y}^{2}\right) \leq C_{d}^{(2 n)} \int_{\mathbb{R}^{d}} e^{-\frac{K_{d}}{2}\left(\|x\|^{d}+\|y-x\|^{d}\right)} d x,
\end{gathered}
$$

$\mathrm{e}$

$$
\mathbb{P}\left(S \in \cap_{k=1}^{n} A_{y_{k}}^{2}\right) \leq C_{d}^{(2 n)} \prod_{k=1}^{n}\left(\int_{\mathbb{R}^{d}} e^{-\frac{K_{d}}{2 n}\left(\|x\|^{d}+\left\|y_{k}-x\right\|^{d}\right)} d x\right) .
$$

e concluir então que para todo $n \geq 0$

$$
\mathbb{E}\left[\left|V_{2}\right|^{n}\right]<\infty
$$

Na próxima seção começaremos a construir uma estrutura mais formal para o trabalho com processos pontuais. Como primeiro passo vamos definir o espaço mensurável onde vivem tais processos. Nosso principal objetivo neste capítulo é resumir os principais resultados relativos à teoria de processos pontuais, de modo a possibilitar a leitura do capítulo principal deste trabalho. Por esta razão não traremos as demonstrações da maior parte dos resultados aqui descritos. O leitor interessado em maiores detalhes pode procurar em [6] e [7].

\subsection{O Espaço das Medidas de Contagem}

Seja $\mathcal{X}$ um espaço métrico completo separável (e.m.c.s.) com $\sigma$-álgebra de Borel $\mathcal{B}(\mathcal{X})$. Diremos que uma medida $\xi \mathrm{em}(\mathcal{X}, \mathcal{B}(\mathcal{X}))$ é localmente finita se $\xi(A)<\infty$ para todo $A \in \mathcal{B}(\mathcal{X})$ limitado. Denote agora por $\mathcal{N}(\mathcal{X})$ o espaço das medidas de contagem localmente finitas em $(\mathcal{X}, \mathcal{B}(\mathcal{X}))$. Diremos que $\xi \in \mathcal{N}(\mathcal{X})$ é simples se

$$
\xi(\{x\}) \leq 1 \text {, para todo } x \in \mathcal{X},
$$

e denotaremos por $\mathcal{N}^{s}(\mathcal{X})$ o espaço das medidas de contagem simples localmente finitas em $(\mathcal{X}, \mathcal{B}(\mathcal{X}))$. Para acomodar melhor a definição de processos pontuais marcados, dado um e.m.c.s $M$ defina o espaço $\mathcal{N}^{m}(\mathcal{X} \times M)$ como o espaço das medidas localmente finitas em $(\mathcal{X} \times M, \mathcal{B}(\mathcal{X} \times M))$ com a 
propriedade adicional de que para cada $\bar{\xi} \in \mathcal{N}^{m}(\mathcal{X} \times M)$ a medida base definida por

$$
\xi(A)=\bar{\xi}(A \times M), \quad A \in \mathcal{B}(\mathcal{X})
$$

é um elemento de $\mathcal{N}(\mathcal{X})$.

É uma propriedade das medidas de contagem localmente finitas que $\xi \in \mathcal{N}(\mathcal{X})$, se e somente se, existe um conjunto enumerável $\left\{\left(x_{i}, k_{i}\right)\right\} \subset \mathcal{X} \times \mathbb{Z}^{+}$, com $\left\{x_{i}\right\}$ localmente finito e tal que

$$
\xi=\sum_{i} k_{i} \delta_{x_{i}}
$$

onde $\delta$ é a medida de Dirac. Observe que se $\xi$ é simples então $k_{i}=1$ para todo $i$ e portanto

$$
\xi=\sum_{i} \delta_{x_{i}}
$$

O conjunto $\left\{x_{i}\right\}$ é chamado de suporte da medida $\xi(\operatorname{supp}(\xi))$. Ao longo deste trabalho nos concentraremos em medidas $\bar{\xi} \in \mathcal{N}^{m}(\mathcal{X} \times M)$ cuja medida de base $\xi$ é simples. Neste caso podemos escrever

$$
\bar{\xi}=\sum_{j} \delta_{\left(x_{j}, m_{j}\right)}
$$

onde $m_{j} \in M$ é a marca associada ao ponto $x_{j}$.

Seja $d$ a métrica em $\mathcal{X}$, e $B_{r}(x)$ a bola aberta em $\mathcal{X}$ de centro em $x \in \mathcal{X}$ e raio $r>0$. Agora, dado $F \subset \mathcal{X}$ e $\epsilon>0$ defina

$$
F^{\epsilon}:=\bigcup_{x \in F} B_{\epsilon}(x)
$$

Seguindo a mesma construção dada no Apêndice em [6], dadas duas medidas $\mu$ e $\nu$ finitas em $\mathcal{X}$, defina

$$
d_{p}(\mu, \nu):=\inf \left\{\epsilon \geq 0: \text { para todo } F \subseteq \mathcal{X} \text { fechado, } \mu(F) \leq \nu\left(F^{\epsilon}\right)+\epsilon \text { e } \nu(F) \leq \mu\left(F^{\epsilon}\right)+\epsilon\right\} .
$$

Fixado $x_{0} \in \mathcal{X}$, faça $B_{r}=B_{r}\left(x_{0}\right)$ e para uma $\mu, \nu \in \mathcal{N}(\mathcal{X})$ denote por $\mu_{r}$ e $\nu_{r}$ as restrições (finitas) de $\mu$ e $\nu$ a $B_{r}$. Agora faça

$$
d_{l f}(\mu, \nu):=\int_{0}^{\infty} e^{-r} \frac{d_{p}\left(\mu_{r}, \nu_{r}\right)}{1+d_{p}\left(\mu_{r}, \nu_{r}\right)} d r
$$

Pode-se mostrar que $d_{l f}$ define uma métrica em $\mathcal{N}(\mathcal{X})$ para a qual $\mathcal{N}(\mathcal{X})$ é completo e separável. Mais do que isso, a $\sigma$-álgebra de Borel $\mathcal{B}(\mathcal{N}(\mathcal{X}))$ coincide com a menor $\sigma$-álgebra que torna mensurável as funções do tipo $\xi \mapsto \xi(B)$, com $B \in \mathcal{B}(\mathcal{X})$ limitado. Ou seja, $\mathcal{B}(\mathcal{N}(\mathcal{X}))$ é a $\sigma$-álgebra gerada por 
eventos do tipo

$$
\left\{\xi \in \mathcal{N}(\mathcal{X}): \xi\left(B_{i}\right)=k_{i} ; i=1, \ldots, n\right\},
$$

onde $B_{i}, i=1, \ldots, n$ são borelianos limitados de $\mathbb{R}^{d}$ e $k_{i}$ são inteiros positivos.

Trabalharemos então no espaço mensurável $(\mathcal{N}(\mathcal{X}), \mathcal{B}(\mathcal{N}(\mathcal{X})))$. Deste ponto em diante, a não ser que se faça necessário, ocultaremos a dependência do espaço $\mathcal{X}$ e notaremos apenas $(\mathcal{N}, \mathcal{B}(\mathcal{N}))$.

Estamos prontos agora para definir o que entenderemos por Processo Pontual.

Definição 2.2. $\quad$ (i) Um Processo Pontual em um e.m.c.s. $\mathcal{X}$ é uma função mensurável de um espaço de probabilidade $(\Omega, \mathcal{F}, \mathbb{P})$ em $(\mathcal{N}(\mathcal{X}), \mathcal{B}(\mathcal{N}(\mathcal{X})))$.

(ii) Um Processo Pontual Marcado em $\mathcal{X}$ com marcas em $M$ é uma função mensurável de um espaço de probabilidade $(\Omega, \mathcal{F}, \mathbb{P})$ em $\left(\mathcal{N}^{m}(\mathcal{X} \times M), \mathcal{B}\left(\mathcal{N}^{m}(\mathcal{X} \times M)\right)\right)$

Diremos que um Processo Pontual $S$ é simples se $\mathbb{P}\left(S \in \mathcal{N}^{s}(\mathcal{X})\right)=1$. No caso de um processo pontual marcado $\bar{S}$, o processo pontual dado por

$$
S(A)=\bar{S}(A \times M), \quad A \in \mathcal{B}(\mathcal{X})
$$

será chamado de processo base de $\bar{S}$.

Observação 2.2.1. Todo processo pontual $S$ em $\mathcal{X}$ pode ser escrito como um processo pontual marcado $\bar{S}$ em $\mathcal{X} \times \mathbb{Z}^{+}$, considerando a multiplicidade $k_{i}$ de cada ponto $x_{i}$ como uma marca, $e$ fazendo o processo de base $\bar{S}$ coincidir com o suporte de $S$.

Observação 2.2.2. Por (2.5), toda medida simples $\xi \in \mathcal{N}\left(\mathbb{R}^{d}\right)$ pode ser identificada com o seu suporte $\operatorname{supp}(\xi)$. Por esta razão, vários autores trabalham apenas no espaço das configurações de pontos localmente finitas, e tratam processos não simples como processos pontuais marcados (Observação 2.2.1). Deste modo, sempre que $S$ for um processo pontual simples, usaremos esta identificação $e$ trataremos indiscriminadamente $S$ como uma medida de contagem ou como uma configuração de pontos. Assim, dentre outras notações, $v \in S$ será o mesmo que $v \in \operatorname{supp}(S)$.

Deste ponto em diante, consideraremos apenas processos pontuais simples (marcados ou não).

Exemplo 2.1. Um exemplo simples de processo pontual é obtido da seguinte maneira. Dada uma v.a. $U$ uniformemente distribuída em $\left[-\frac{1}{2}, \frac{1}{2}\right]^{d}$, podemos construir um processo pontual $S^{\circ}$ como

$$
S^{\mathrm{o}}=\left\{z+U: z \in \mathbb{Z}^{d}\right\}
$$


Exemplo 2.2 (Processos de Cox). Seja $Z:=\left\{Z_{x} ; x \in \mathbb{R}^{d}\right\}$ um campo aleatório positivo com

$$
\mathbb{E}\left[\int_{B} Z_{x} d x\right]<\infty
$$

para todo $B \in \mathcal{B}\left(\mathbb{R}^{d}\right)$ limitado. O Processo de Cox determinado por $Z$ é um o processo pontual $S^{\text {o }}$ tal que, para cada realização de $Z, S^{\circ}$ é um processo de Poisson com função intensidade dada por $Z$. Ou seja, $S^{\circ}$ é um processo de Poisson com função intensidade aleatória dada por $\left\{Z_{x} ; x \in \mathbb{R}^{d}\right\}$

Outros exemplos de processos pontuais podem ser encontrados em [22], [6] e [7]. Em [13], por exemplo, os autores constroem, via um algoritmo de simulação perfeita, processos pontuais com lei absolutamente contínua em relação a lei de um processo de Poisson homogêneo.

\subsection{Processos Pontuais Estacionários}

Vamos nos concentrar agora em processos pontuais (marcados ou não) com pontos em $\mathbb{R}^{d}$. Assim, para $x \in \mathbb{R}^{d}$ denote por $\tau_{x}: \mathbb{R}^{d} \rightarrow \mathbb{R}^{d}$ a translação usual em $\mathbb{R}^{d}$ dada por $\tau_{x}(y)=y+x$. Abusando da notação, notaremos também por $\tau_{x}$ a extensão para $\mathcal{N}\left(\mathbb{R}^{d}\right)$ dada por

$$
\left(\tau_{x} \xi\right)(B):=\xi\left(\tau_{x} B\right)
$$

onde $B$ é um boreliano em $\mathbb{R}^{d}$, e $\tau_{x} B=\{x+y: y \in B\}$. Em outras palavras, $\tau_{x} \xi$ é a medida de contagem com suporte em $\{v-x: v \in \operatorname{supp}(\xi)\}$

Se $\bar{\xi} \in \mathcal{N}\left(\mathbb{R}^{d} \times M\right)$ é dado por

$$
\bar{\xi}=\sum_{j} \delta_{\left(x_{j}, m_{x_{j}}\right)}
$$

definiremos $\tau_{x} \bar{\xi}$ por

$$
\tau_{x} \bar{\xi}=\sum_{j} \delta_{\left(x_{j}-x, m_{x_{j}}\right)}
$$

Neste contexto, diremos que um processo pontual $S$ (marcado ou não) é estacionário se a lei $\mu$ de $S$ for invariante por ações do grupo $\left\{\tau_{x}, x \in \mathbb{R}^{d}\right\}$, ou seja, se para todo $x \in \mathbb{R}^{d}$ e $U \in \mathcal{B}(\mathcal{N})$ vale que

$$
\mu(U):=\mathbb{P}(S \in U)=\mathbb{P}\left(\tau_{x} S \in U\right) .
$$

Um exemplo de processo estacionário é o processo de Poisson homogêneo, definido na seção anterior. Outros exemplos são o processo construído no Exemplo 2.1, e o processo de Cox (Exemplo 2.2) definido por um campo $Z$ estacionário.

Definição 2.3. Dado um Processo Pontual $S$, a medida de intensidade de $S$ é a função de conjuntos 
$\lambda: \mathcal{B}\left(\mathbb{R}^{d}\right) \rightarrow \mathbb{R}^{+}$dada por

$$
\lambda(A):=\mathbb{E}[S(A)] .
$$

Proposição 2.4. $\lambda$ é uma medida $\left(\mathbb{R}^{d}, \mathcal{B}\left(\mathbb{R}^{d}\right)\right)$. Se $S$ é estacionário e tal que $\lambda(A)<\infty$ para todo $A \in \mathcal{B}\left(\mathbb{R}^{d}\right)$ limitado, então $\lambda(\cdot)=\rho \ell(\cdot)$ para algum $\rho>0$.

Demonstração. A $\sigma$-aditividade de $\lambda$ segue da $\sigma$-aditividade de $S$. Tomando uma sequência crescente de conjuntos $A_{n} \nearrow A$, segue do teorema da convergência monótona que $\lambda\left(A_{n}\right) \nearrow \lambda(A)$. E assim $\lambda$ é contínua inferiormente e portanto uma medida.

No caso estacionário, $\lambda$ é claramente invariante por translações no $\mathbb{R}^{d}$. Se além disso for localmente finita, só pode ser múltiplo da medida de Lebesgue.

A medida $\lambda$ é também chamada de medida de primeiro momento do processo $S$. No caso em que $\lambda$ é localmente finita, dizemos que que $S$ tem primeiro momento finito. Tal conceito pode ser facilmente estendido para outros momentos.

Definição 2.5. Definimos medida de momento de ordem $k$ de um Processo Pontual $N$ como a medida $\lambda^{(k)}$ em $\left(\mathbb{R}^{d}\right)^{k}$ dada por

$$
\lambda^{(k)}\left(A_{1} \times \cdots \times A_{k}\right):=\mathbb{E}\left[\left|N \cap A_{1}\right| \cdots\left|N \cap A_{k}\right|\right] .
$$

Argumentos usuais agora nos mostram como integrar com relação à $\lambda^{(k)}$, e como consequência temos uma maneira de calcular momentos de somas aleatórias. Este é o conteúdo do lema a seguir.

Lema 2.6. Se $f:\left(\mathbb{R}^{d}\right)^{k} \rightarrow \mathbb{R}$ é limitada e tem suporte limitado então

$$
\mathbb{E}\left[\sum_{x_{i} \in S, i=1, \ldots, k} f\left(x_{1}, \ldots, x_{k}\right)\right]=\int_{\left(\mathbb{R}^{d}\right)^{k}} f d \lambda^{(k)},
$$

em particular

$$
\mathbb{E}\left[\left(\sum_{v \in S} h(v)\right)^{k}\right]=\int_{\left(\mathbb{R}^{d}\right)^{k}} h\left(x_{1}\right) \cdots h\left(x_{k}\right) \lambda^{(k)}\left(d x_{1} \times \cdots \times d x_{k}\right),
$$

para toda $h: \mathbb{R} \rightarrow \mathbb{R}$ limitada com suporte limitado.

Demonstração. No caso $k=1$ basta seguir os argumentos tradicionais de indicadoras, para funções simples, para limites monótonos de funções simples.

Para os outros casos definimos o processo pontual $S_{k}$ em $\left(\mathbb{R}^{d}\right)^{k}$ por $S_{k}\left(A_{1} \times \cdots \times A_{k}\right)=$ $S\left(A_{1}\right) \cdots S\left(A_{k}\right)$, e daí é só observar que $\lambda^{(k)}$ é apenas a medida intensidade do processo $S_{k}$ e o resultado segue do caso $k=1$. 
Para obter (2.10) basta fazer $f\left(x_{1}, \cdots, x_{k}\right)=\prod_{i=1}^{k} h\left(x_{i}\right)$ em $(2.9)$.

Teorema Ergódico Espacial Antes de continuarmos com o estudo de processos pontuais estacionários, faremos um breve parentese para enunciar um importante resultado na teoria de processos espaciais: o Teorema Ergódico Espacial. Provado em 1972 (ver [26]), este teorema é a versão espacial $(d>1)$ do importante Teorema Ergódico de Birkhoff (ver [9]).

Considere então um espaço mensurável $(\Omega, \mathcal{F})$, onde esteja definido um grupo de transformações mensuráveis $\omega \mapsto T_{x} \omega$ indexado por $x \in \mathbb{R}^{d}$. No contexto tratado até agora, podemos considerar o grupo $\left\{\tau_{x} ; x \in R^{d}\right\}$ agindo sobre $(\mathcal{N}, \mathcal{B}(\mathcal{N}))$.

Neste contexto, diremos que uma medida de probabilidade $\mathbb{P}$ definida em $(\Omega, \mathcal{F})$ é invariante por ações do grupo se $T_{x} \mathbb{P}=\mathbb{P}$ para todo $x \in \mathbb{R}^{d}$. Diremos que um evento $U \in \mathcal{F}$ é invariante pela ação do grupo se $\mathbb{P}\left(T_{x} U \triangle U\right)=0$ para todo $x \in \mathbb{R}^{d}$. É um exercício simples verificar que a família de eventos $\mathcal{I}=\{U \in \mathcal{F}: U$ é invariante por ações do grupo $\}$ forma uma $\sigma$-álgebra. Continuando a analogia com processos pontuais, consideraremos a distribuição $\mu$ em $(\mathcal{N}, \mathcal{B}(\mathcal{N}))$ de um processo pontual estacionário, e neste caso diremos que tanto a medida, quanto os eventos de $\mathcal{I}$, são invariantes por translações.

Antes de enunciarmos o teorema ergódico, precisamos de uma última definição.

Definição 2.7. Diremos que $\left\{A_{n} ; n \geq 0\right\} \subset \mathcal{B}\left(\mathbb{R}^{d}\right)$ é uma sequência convexa mediadora em $\mathbb{R}^{d}$ se

(i) $A_{n}$ é convexo para todo $n \geq 0$;

(ii) $A_{n} \subseteq A_{n+1}$ para todo $n \geq 0$;

(iii) $r\left(A_{n}\right):=\sup \left\{r: A_{n}\right.$ contém uma bola de raio $\left.r\right\} \rightarrow \infty$ quando $n \rightarrow \infty$.

Teorema 2.8 (Teorema Ergódico Espacial). Seja $(\Omega, \mathcal{F}, \mathbb{P})$ um espaço de probabilidade, $\mathcal{G}=\left\{T_{x} ; x \in\right.$ $\left.\mathbb{R}^{d}\right\}$ um grupo de transformações em $\Omega$ tal que $\mathbb{P}$ é invariante por ações de $\mathcal{G}$, e $\left(\Lambda_{n} ; n \geq 0\right) \subset \mathcal{B}\left(\mathbb{R}^{d}\right)$ uma sequência convexa mediadora. Nestas condições vale que para toda $f \in L_{1}(\mathbb{P})$,

$$
\lim _{n \rightarrow \infty} \frac{1}{\ell\left(\Lambda_{n}\right)} \int_{\Lambda_{n}} f\left(T_{x} \omega\right) d x=\mathbb{E}[f \mid \mathcal{I}], \quad \text { q.c.. }
$$

\subsubsection{Processos Pontuais Ergódicos e Mixing}

Nesta seção definiremos processos pontuais ergódicos e mixing, e enunciaremos os resultados mais importantes relacionados a tais processos. O leitor interessado em maiores detalhes e demonstrações dos resultados descritos abaixo pode procurar, por exemplo, o livro [7], que traz em detalhes tudo o que será descrito aqui. 
Para facilitar a notação, deste ponto até o final do trabalho, dado um processo estacionário $S$, denotaremos por $\mu$ a lei de $S$ em $(\mathcal{N}, \mathcal{B}(\mathcal{N}))$. Por ora não faremos distinção entre processos marcados ou não.

Seja $S$ um processo pontual estacionário (marcado ou não) em $\mathbb{R}^{d}$ e $\left\{A_{n} ; n \geq 0\right\}$ uma sequência convexa mediadora em $\mathbb{R}^{d}$. Diremos que $\mu$ é

(i) ergódica para o grupo $\left\{\tau_{x} ; x \in \mathbb{R}^{d}\right\}$, ou simplesmente que $S$ é ergódico, se

$$
\lim _{n \rightarrow \infty} \frac{1}{\ell\left(A_{n}\right)} \int_{A_{n}}\left[\mu\left(U_{1} \cap \tau_{x} U_{2}\right)-\mu\left(U_{1}\right) \mu\left(U_{2}\right)\right] d x=0
$$

para todo $U_{1}, U_{2} \in \mathcal{B}(\mathcal{N})$

(ii) mixing para o grupo $\left\{\tau_{x} ; x \in \mathbb{R}^{d}\right\}$, ou simplesmente que $S$ é mixing, se

$$
\lim _{\|x\| \rightarrow \infty}\left|\mu\left(U_{1} \cap \tau_{x} U_{2}\right)-\mu\left(U_{1}\right) \mu\left(U_{2}\right)\right|=0
$$

para todo $U_{1}, U_{2} \in \mathcal{B}(\mathcal{N})$

É fácil ver que se $S$ é mixing então $S$ é ergódico, mas o inverso pode não ser verdade. Exemplos naturais podem ser encontrados entre os chamados processo periódicos, que definimos a seguir. Para isso, considere o evento

$$
\mathcal{M}=\left\{\xi \in \mathcal{N}: \exists x \in \mathbb{R}^{d} \text { tal que } \tau_{x} \xi=\xi, x \neq 0\right\}
$$

Diremos que um processo pontual é $S$ é periódico se $\mathbb{P}(S \in \mathcal{M})=1$. O processo construído no Exemplo 2.1 é um exemplo de processo periódico. Este é também um exemplo de processo ergódico, mas não mixing.

Observe agora que, se $S$ é um processo pontual ergódico com lei $\mu$ e $U \in \mathcal{I}$, a $\sigma$-álgebra dos eventos invariantes por translação, então, pela equação (2.11), temos que

$$
\mu(U)-\mu(U)^{2}=\frac{1}{\ell\left(A_{n}\right)} \int_{A_{n}}\left[\mu(U \cap U)-\mu(U)^{2}\right] d x=\frac{1}{\ell\left(A_{n}\right)} \int_{A_{n}}\left[\mu\left(U \cap \tau_{x} U\right)-\mu(U)^{2}\right] d x \rightarrow 0,
$$

de onde segue que $\mu(U)=\mu(U)^{2}$ e assim $\mu(U) \in\{0,1\}$. Por outro lado, se $\mathcal{I}$ é trivial, fazendo $f:=\mathbf{1}_{U_{1}}$ temos que $f \circ \tau_{x}=\mathbf{1}_{\tau_{-x} U_{1}}$ e do Teorema 2.8

$$
\frac{1}{\ell\left(A_{n}\right)} \int_{A_{n}} \mathbf{1}_{\left\{\tau_{x} U_{1} \cap U_{2}\right\}} d x=\frac{1}{\ell\left(A_{n}\right)} \mathbf{1}_{U_{2}} \int_{A_{n}} f \circ \tau_{-x} d x \rightarrow \mathbf{1}_{U_{2}} \mu\left(U_{1}\right),
$$


$\mu$-quase-certamente. Integrando com relação a $\mu$, pelo teorema da convergência dominada, obtemos então que

$$
\frac{1}{\ell\left(A_{n}\right)} \int_{A_{n}}\left[\mu\left(U_{1} \cap \tau_{x} U_{2}\right)-\mu\left(U_{1}\right) \mu\left(U_{2}\right)\right] d x \rightarrow 0,
$$

de onde concluímos que $\mu$ é ergódica. Mostramos assim que ergodicidade é equivalente a $\sigma$-álgebra invariante $\mathcal{I}$ ser trivial.

Do mesmo modo, dizer que um processo pontual $S$ é mixing é equivalente a $\sigma$-álgebra $\mathcal{J}$ de eventos caudais para $S$ é trivial. Para definir esta $\sigma$-álgebra, dado $n \geq 0$ denote por $\mathcal{J}_{n}$ a menor $\sigma$-álgebra para a qual $S(A), A \in \mathcal{B}\left(\mathbb{R}^{d} \backslash[-n, n]^{d}\right)$ é mensurável, ou seja, a $\sigma$-álgebra determinada pelo comportamento de $S$ fora de $[-n, n]^{d}$. A $\sigma$-álgebra caudal é definida então como a intersecção de todas as $\mathcal{J}_{n}$, isto é,

$$
\mathcal{J}=\bigcap_{n \geq 0} \mathcal{J}_{n}
$$

Em geral, $\mathcal{J}$ é maior que $\mathcal{I}$. Como não teremos necessidade de tais conceitos, não nos estenderemos mais no assunto. $\mathrm{O}$ leitor interessado em maiores detalhes sobre a relação entre mixing e a trivialidade de $\mathcal{J}$ deve ver [7].

\subsection{Medidas de Palm}

Nesta seção introduziremos a chamada medida de Palm associada a um processo pontual. Esta medida representa a distribuição do processo visto de um ponto típico, ou ainda, de um ponto escolhido ao acaso. Tais interpretações ficarão mais claras após a definição que daremos a seguir. Apesar de poderem ser definidas em um contexto geral, as principais aplicações de tais medidas estão no estudo de processos pontuais estacionários. Por esta razão nos concentraremos em dar sua definição apenas nesta classe de processos.

Dado um processo pontual estacionário $S$, seja $\nu: \mathcal{B}\left(\mathbb{R}^{d} \times \mathcal{N}\right) \rightarrow[0,+\infty]$ a medida dada por

$$
\nu(A \times U)=\mathbb{E}\left[\sum_{v \in S \cap A} \mathbf{1}_{U}\left(\tau_{v} S\right)\right] .
$$


Note que para cada $x \in \mathbb{R}^{d}$ e $U \in \mathcal{B}(\mathcal{N})$ fixos

$$
\begin{aligned}
\nu\left(\tau_{x} A \times U\right) & =\mathbb{E}\left[\sum_{v \in S \cap \tau_{x} A} \mathbf{1}_{U}\left(\tau_{v} S\right)\right] \\
& =\mathbb{E}\left[\sum_{v \in \tau_{x} S \cap A} \mathbf{1}_{U}\left(\tau_{v} \tau_{x} S\right)\right] \\
& =\mathbb{E}\left[\sum_{v \in S \cap A} \mathbf{1}_{U}\left(\tau_{v} S\right)\right] \\
& =\nu(A \times U),
\end{aligned}
$$

Deste modo, para cada $U \in \mathcal{B}(\mathcal{N})$ existe uma constante $\hat{\nu}(U)$ tal que

$$
\nu(A \times U)=\hat{\nu}(U) \ell(A) .
$$

Não é difícil mostrar que $\hat{\nu}$ é uma medida em $(\mathcal{N}, \mathcal{B}(\mathcal{N}))$. Além disso, se $S$ tem intensidade $\lambda<\infty$ então $\nu(A \times \mathcal{N})=\lambda \ell(A)$ e portanto

$$
\hat{\nu}(\mathcal{N})=\lambda
$$

e assim podemos definir uma medida de probabilidade $\mu_{\mathrm{o}}$ em $(\mathcal{N}, \mathcal{B}(\mathcal{N}))$ por

$$
\mu_{\mathrm{o}}(\cdot):=\frac{1}{\lambda} \hat{\nu}(\cdot)
$$

A medida $\mu_{\mathrm{o}}$ que acabamos de definir é conhecida como Medida de Palm associada ao processo estacionário $S$. O processo pontual com lei $\mu_{\mathrm{o}}$ será denotado por $S^{\mathrm{o}}$ e denominado de versão Palm de $S$.

Em outras palavras, $\mu_{\mathrm{o}}$ é a medida em $\mathcal{B}(\mathcal{N})$ dada por

$$
\mu_{\mathrm{o}}(U):=\frac{1}{\lambda} \mathbb{E}\left[\sum_{v \in S \cap C_{1}} \mathbf{1}_{U}\left(\tau_{v} S\right)\right],
$$

onde $C_{1}=\left[-\frac{1}{2}, \frac{1}{2}\right]^{d}$, e $U \in \mathcal{B}(\mathcal{N})$.

Observe que a definição acima é válida para processos estacionários marcados ou não. Neste caso, se $\bar{S}$ é um processo marcado estacionário denotaremos por $\bar{\mu}$ sua lei, por $\bar{\mu}_{\mathrm{o}}$ a medida de Palm associada e $\bar{S}$, e por $\bar{S}^{o}$ sua versão de Palm. Uma inspeção mais cuidadosa da definição acima mostra que, se $\overline{S^{\circ}}$ possui marcas independentes (do processo e entre si), então as marcas em $\bar{S}^{\text {o }}$ tem a mesma propriedade. 
É importante observar que a medida $\mu_{\mathrm{o}}$ só enxerga configurações com um ponto na origem. De fato, definindo

$$
\mathcal{N}^{\circ}:=\{\xi \in \mathcal{N}: 0 \in \xi\}
$$

temos que se $\xi \in \mathcal{N}$ então para todo $x \in \mathbb{R}^{d}$ temos que $\tau_{x} \xi \in \mathcal{N}$, em particular, se $x \in \xi$ vale também que $\tau_{x} \xi \in \mathcal{N}^{\circ}$. E assim, por (2.13)

$$
\begin{aligned}
\mu_{\mathrm{o}}\left(\mathcal{N}^{\mathrm{o}}\right) & =\frac{1}{\lambda} \mathbb{E}\left[\sum_{v \in S \cap C_{1}} \mathbf{1}_{\mathcal{N}^{\circ}}\left(\tau_{v} S\right)\right] \\
& =\frac{1}{\lambda} \mathbb{E}\left[\left|S \cap C_{1}\right|\right] \\
& =1
\end{aligned}
$$

Da mesma forma, vale que

Proposição 2.9. Se $S$ é um processo pontual estacionário e $\Gamma \in \mathcal{B}(\mathcal{N})$ um evento invariante por translações com $\mu(\Gamma)=1$, então

$$
\mu_{\mathrm{o}}\left(\Gamma_{\mathrm{o}}\right)=1
$$

onde $\Gamma_{\mathrm{o}}=\Gamma \cap \mathcal{N}^{\mathrm{o}}$.

Demonstração. Como argumentado acima, pela invariância por translações de $\Gamma$, se $\xi \in \Gamma$ então para todo $x \in \mathbb{R}^{d}$ temos que $\tau_{x} \xi \in \Gamma$, em particular, se $x \in \xi$ vale também que $\tau_{x} \xi \in \mathcal{N}^{\circ}$ e portanto $\tau_{x} \xi \in \Gamma_{\mathrm{o}}$. Assim, pela definição de $\mu_{\mathrm{o}}$ vale que

$$
\begin{aligned}
\mu_{\mathrm{o}}\left(\Gamma_{\mathrm{o}}\right) & =\frac{1}{\lambda} \mathbb{E}\left[\sum_{v \in S \cap C_{1}} \mathbf{1}_{\Gamma_{\mathrm{o}}}\left(\tau_{x} S\right)\right] \\
& =\frac{1}{\lambda} \int_{\Gamma_{v \in S \cap C_{1}}} \mathbf{1}_{\Gamma_{\mathrm{o}}}\left(\tau_{x} \xi\right) \mu(d \xi) \\
& =\frac{1}{\lambda} \int_{\Gamma}\left|\xi \cap C_{1}\right| \mu(d \xi) \\
& =1
\end{aligned}
$$

Vamos agora enumerar algumas das diferentes caracterizações para medida de Palm encontradas na literatura. Todas as caracterizações listadas a seguir podem ser encontradas em [7] ou [27].

A proposição abaixo, cuja demonstração pode ser encontrada em [7], justifica a interpretação de que a medida de Palm é dada pela distribuição do processo, condicionada a haver um ponto na 
origem.

Proposição 2.10. Se $S$ é um processo pontual estacionário e $v^{*} \in S$ é o ponto do processo mais próximo da origem, então para toda $f: \mathcal{N} \rightarrow \mathbb{R}$ mensurável, positiva e limitada

$$
\mathbb{E}\left[f\left(S^{\mathrm{o}}\right)\right]=\lim _{r \rightarrow 0} \mathbb{E}\left[f\left(\tau_{v^{*}} S\right) \mid S\left(B_{r}\right)>0\right],
$$

onde $B_{r} \in \mathbb{R}^{d}$ é a bola de raio $r$ e centro na origem.

No caso em que $S$ é um processo de Poisson, uma simples aplicação da fórmula de SlivniackMecke (Proposição 2.1), mostra que $S^{\text {o }}$ pode ser construído a partir de $S$ simplesmente adicionando um ponto na origem. Ou seja, o processo $S+\delta_{0}$ é distribuído de acordo com a medida de Palm associada a $S$. Mais do que isso, pode-se mostrar que se $S+\delta_{0}$ tem lei de Palm associada com algum processo $S$, então $S$ é necessariamente um processo de Poisson, de modo que apenas o processo de Poisson tem esta característica.

A próxima Proposição, que pode ser encontrada em [7] e [27], relaciona as medidas $\mu$ e $\mu_{\mathrm{o}}$ através das células de Voronoi.

Proposição 2.11. Se $S$ é estacionário com intensidade finita $\lambda$, então para toda $f: \mathcal{N} \rightarrow \mathbb{R}$ mensurável

$$
\mathbb{E}[f(S)]=\lambda \mathbb{E}\left[\ell(\mathrm{C}(0, \xi)) f\left(\tau_{U} S^{\mathrm{o}}\right)\right],
$$

onde, condicionado a $S^{\mathrm{o}}, U$ é distribuido uniformemente em $\mathrm{C}\left(0, S^{\mathrm{o}}\right)$.

Como aplicação direta da Proposição anterior concluímos que

$$
\mathbb{E}\left[\ell\left(\mathrm{C}\left(0, S^{\mathrm{o}}\right)\right)\right]=\frac{1}{\lambda} .
$$

O próximo resultado caracteriza o processo $S$ como o limite assintótico de $\tau_{x} S^{\text {o }}$. Em palavras, a medida que nos afastamos da origem o processo $S^{\circ}$, mais o processo se aproxima do equilibrio, se parecendo mais com o processo estacionário $S$. Isso é claro para processos de Poisson, uma vez que que podemos acoplar $S^{\circ}$ e $S$ fazendo $S^{\circ}=S+\delta_{0}$.

Proposição 2.12. Se $S$ é mixing então para toda $f: \mathcal{N} \rightarrow \mathbb{R}$ mensurável e limitada

$$
\lim _{\|x\| \rightarrow \infty} \mathbb{E}\left[f\left(\tau_{x} S^{\mathrm{o}}\right)\right]=\mathbb{E}[f(S)]
$$

Corolário 2.13. Se $S$ é mixing, então $\tau_{x} S^{\circ}$ converge fraco para $S$ quando $\|x\| \rightarrow \infty$. 
A seguir enunciaremos dois importantes resultados relacionados à teoria de Palm para processos pontuais estacionários. O primeiro, conhecido como Fórmula de Campbell, pode ser demonstrado utilizando técnicas padrão da teoria de medidas. O segundo determina as propriedades ergódicas de $\mu_{\mathrm{o}}$, e é consequência (não tão direta) do Teorema 2.8, além de reforçar a intrepetação de que a medida de Palm representa a lei do processo visto a partir de um ponto escolhido ao acaso. A demonstração de ambos pode ser encontrada em [7].

Proposição 2.14 (Fórmula de Campbell). Se $S^{\mathrm{o}}$ é a versão de Palm de um processo pontual estacionário $S$ de intensidade $\lambda<\infty$, então

$$
\mathbb{E}\left[\sum_{v \in S} f(v, S)\right]=\lambda \int \mathbb{E}\left[f\left(x, \tau_{-x} S^{\mathrm{o}}\right)\right] d x .
$$

Equivalentemente

$$
\mathbb{E}\left[\sum_{v \in S} f\left(v, \tau_{v} S\right)\right]=\lambda \int \mathbb{E}\left[f\left(x, S^{\mathrm{o}}\right)\right] d x .
$$

Proposição 2.15 (Teorema Ergódico Pontual). Se S é um processo pontual ergódico com intensidade $\lambda<\infty$ e $f \in L_{1}\left(\mathcal{N}, \mu_{\mathrm{o}}\right)$, então para toda sequência convexa mediadora $\left\{A_{n} ; n \geq 1\right\}$

$$
\lim _{n \rightarrow \infty} \frac{1}{\ell\left(A_{n}\right)} \sum_{v \in S^{\circ} \cap A_{n}} f\left(\tau_{v} S\right)=\lambda \mathbb{E}\left[f\left(S^{\mathrm{o}}\right)\right] \quad \text { q.c. }
$$

O Princípio de Transporte de Massa Outro resultado importante relacionado com a teoria de Palm em processos pontuais é o Princípio de Transporte de Massa (PTM) que, de maneira simplificada, diz que para processos pontuais estacionários $S$, "massa total média que deixa a origem em $S^{\text {o }}$ é igual a massa média que entra na origem". A "massa" a qual nos referimos será dada por uma função $g: \mathbb{R}^{d} \times \mathbb{R}^{d} \times \mathcal{N}\left(\mathbb{R}^{d}\right) \rightarrow \mathbb{R}$, onde para $x, y \in \xi, g(x, y, \xi)$ representa a massa transportada entre $x$ e $y$. Diremos que $g$ é covariante se para todo $x, y, z \in \xi$ vale que

$$
g(x, y, \xi)=g\left(x-z, y-z, \tau_{z} \xi\right) .
$$

Mais precisamente, vale que

Lema 2.16 (Princípio de Transporte de Massa). Seja $S$ um processo pontual estacionário em $\mathbb{R}^{d}$ com intensidade $\lambda<\infty$. Seja $g: \mathbb{R}^{d} \times \mathbb{R}^{d} \times \mathcal{N}\left(\mathbb{R}^{d}\right) \rightarrow \mathbb{R}$ covariante. Se g é positiva, ou tal que

$$
\mathbb{E}\left[\sum_{v \in S^{\circ}}\left|g\left(0, v, S^{\mathrm{o}}\right)\right|\right]<\infty
$$


então

$$
\mathbb{E}\left[\sum_{v \in S^{\circ}} g\left(0, v, S^{\mathrm{o}}\right)\right]=\mathbb{E}\left[\sum_{v \in S^{\circ}} g\left(v, 0, S^{\mathrm{o}}\right)\right]
$$

Demonstração. Para cada $z_{k} \in \mathbb{Z}^{d}$, seja $C_{z_{k}}=\left[-\frac{1}{2}, \frac{1}{2}\right]^{d}+z_{k}$.

Sem perda de generalidade, considere $\lambda=1$.

Vamos primeiro olhar para o caso em que $g$ é positiva. Da covariância de $g$ segue que

$$
\begin{aligned}
\mathbb{E}\left[\sum_{v \in S^{\circ}} g\left(0, v, S^{\mathrm{o}}\right)\right] & =\mathbb{E}\left[\sum_{v \in S \cap C_{0}} \sum_{w \in \tau_{v} S} g\left(0, w, \tau_{v} S\right)\right] \\
& =\mathbb{E}\left[\sum_{v \in S \cap C_{0}} \sum_{w \in S} g\left(0, w-v, \tau_{v} S\right)\right] \\
& =\mathbb{E}\left[\sum_{v \in S \cap C_{0}} \sum_{w \in S} g(v, w, S)\right] \\
& =\mathbb{E}\left[\sum_{v \in S \cap C_{0}} \sum_{k=0}^{\infty} \sum_{w \in S \cap C_{z_{k}}} g(v, w, S)\right]
\end{aligned}
$$

Agora, da estacionariedade de $S$ e do Teorema da Convergência Monótona, segue que

$$
\begin{aligned}
\mathbb{E}\left[\sum_{v \in S^{\circ}} g\left(0, v, S^{\mathrm{o}}\right)\right] & =\sum_{k=0}^{\infty} \mathbb{E}\left[\sum_{v \in S \cap C_{0}} \sum_{w \in S \cap C_{z_{k}}} g(v, w, S)\right] \\
& =\sum_{k=0}^{\infty} \mathbb{E}\left[\sum_{v \in \tau_{z_{k}} S \cap C_{-z_{k}}} \sum_{w \in \tau_{z_{k}} S \cap C_{0}} g\left(v+z_{k}, w+z_{k}, S\right)\right] \\
& =\sum_{k=0}^{\infty} \mathbb{E}\left[\sum_{v \in \tau_{z_{k}} S \cap C_{-z_{k}}} \sum_{w \in \tau_{z_{k}} S \cap C_{0}} g\left(v, w, \tau_{z_{k}} S\right)\right] \\
& =\sum_{k=0}^{\infty} \mathbb{E}\left[\sum_{w \in S \cap C_{0}} \sum_{v \in S \cap C_{-z_{k}}} g(v, w, S)\right] .
\end{aligned}
$$


Concluimos então que

$$
\begin{aligned}
\mathbb{E}\left[\sum_{v \in S^{\circ}} g\left(0, v, S^{\mathrm{o}}\right)\right] & =\mathbb{E}\left[\sum_{w \in S \cap C_{0}} \sum_{k=0}^{\infty} \sum_{v \in S \cap C_{-z_{k}}} g(v, w, S)\right] \\
& =\mathbb{E}\left[\sum_{w \in S \cap C_{0}} \sum_{v \in S} g(v, w, S)\right] \\
& =\mathbb{E}\left[\sum_{w \in S \cap C_{0}} \sum_{v \in S} g\left(v-w, 0, \tau_{w} S\right)\right] \\
& =\mathbb{E}\left[\sum_{w \in S \cap C_{0}} \sum_{v \in \tau_{w} S} g\left(v, 0, \tau_{w} S\right)\right] \\
& =\mathbb{E}\left[\sum_{v \in S^{\mathrm{o}}} g\left(v, 0, S^{\mathrm{o}}\right)\right] .
\end{aligned}
$$

Para o caso integrável, observamos primeiro que da primeira parte do lema segue que

$$
\mathbb{E}\left[\sum_{v \in S^{\circ}}\left|g\left(v, 0, S^{\mathrm{o}}\right)\right|\right]=\mathbb{E}\left[\sum_{v \in S^{\circ}}\left|g\left(0, v, S^{\mathrm{o}}\right)\right|\right]<\infty
$$

Deste ponto em diante, a demostração segue os mesmos passos do caso positivo, mas agora utilizando o Teorema da Convergência Dominada para trocar a esperança com a soma.

\subsubsection{Shifts Bijetivos e a Estacionariedade Pontual}

Para que possamos expor melhor o conceito que queremos explorar, comecemos com um exemplo simples em $d=1$. Seja $N$ um processo de Poisson estacionário de intensidade 1 em $\mathbb{R}$. Escreva $N=\left\{\ldots, S_{-2}, S_{-1}, S_{0}, S_{1}, S_{2}, \ldots\right\}$, com $\cdots<S_{-1}<0 \leq S_{0}<S_{1}<\cdots$. Considere agora a versão Palm $N^{\mathrm{o}}=N \cup\{0\}$ e enumere os pontos de $N^{\mathrm{o}}$ de modo que $\cdots<S_{-1}<0=S_{0}<S_{1}<\cdots$. Ao fazer isso as variáveis $S_{k}-S_{k-1}$ passam a ser independentes com distribuição Exponencial de média 1 , para todo $k \in \mathbb{Z}$, de modo que os intervalos entre pontos formam agora um processo estacionário.

Observação 2.4.1. O mesmo acontece para qualquer processo pontual estacionário em $\mathbb{R}$. Ou seja, para todo processo estacionário $N$ em $\mathbb{R}$, sua versão Palm é tal que os intervalos entre pontos formam um processo estacionário. Para maiores detalhes ver [6].

Em [27] Thorisson chama esta propriedade de ciclo-estacionariedade (ou estacionariedade por intervalos), e pergunta como fazer para estender esta teoria para $d>1$. Neste caso, a função 
dos intervalos poderiam ser facilmente exercida pelas células de Voronoi, mas a noção de cicloestacionariedade é mais complicada de se formular em $d>1$.

Para fazer o papel da ciclo-estacionariedade em dimensões superiores a 1, Thorisson introduz então a noção de ponto-estacionariedade. Em palavras, seria o mesmo que dizer que um dado processo pontual com um ponto na origem tem a mesma lei visto a partir de qualquer outro ponto do processo. Para $d=1$ isso se resume a dizer que $\tau_{S_{n}} N^{o}$ (o processo $N^{o}$ visto a partir de $S_{n}$ ) tem a mesma distribuição de $N^{o}$. Isso é claro para processos de renovação, mas pode ser estendido para processos pontuais estacionários gerais. $\operatorname{Em} d>1$ também não é completamente claro o que isto significa, uma vez a posição dos pontos é aleatória, e a frase "visto de um ponto do processo" não tem significado preciso.

O problema se resuma agora a encontrar maneiras de se escolher um ponto dentro de um processo pontual. Mas tal escolha não pode ser feita de qualquer maneira. Para que a lei seja mantida, uma vez que translademos da origem para o ponto selecionado, precisamos ser capazes de voltar para o ponto original, isto é, o modo de escolha deve ser "bijetivo".

A seguir vamos fazer um pequeno resumo da teoria de ponto-estacionariedade. Tal teoria, que teve início em [27] teve muitos avanços ao longo dos últimos anos, e um apanhado dos principais resultados pode ser encontrado em [7].

Diremos que uma função $\varphi: \mathcal{N}\left(\mathbb{R}^{d}\right) \rightarrow \mathbb{R}^{d}$ é um mapeamento pontual (ou point-map) se $\varphi(\xi) \in \xi$ sempre que $\xi \in \mathcal{N}^{\circ}$ (ou seja, se $0 \in \xi$ ). Dado um mapeamento pontual segue naturalmente um shift pontual dado por $\xi \mapsto \sigma(\xi):=\tau_{\varphi(\xi)} \xi$. Diremos que tanto o mapeamento pontual $\varphi$ quanto o shift pontual associado $\sigma$ são bijetivos se a função $x \mapsto \psi_{\xi}(x):=\varphi\left(\tau_{x} \xi\right)+x$ é uma bijeção em $\xi$. Neste caso observe que a função inversa de $\psi_{\xi}$ é da forma

$$
\psi_{\xi}^{-1}(x):=\psi_{\tau_{x} \xi}^{-1}(0)+x
$$

O lema abaixo mostra que medidas de Palm associadas a processos pontuais estacionários são invariantes por shifts feitos a partir de mapeamentos pontuais bijetivos. Mais exatamente, vale que

Lema 2.17. Se $S$ é processo pontual estacionário de intensidade $\lambda<\infty$ e $\varphi: \mathcal{N}\left(\mathbb{R}^{d}\right) \rightarrow \mathbb{R}^{d} u m$ mapeamento pontual bijetivo e $\sigma$ o shift pontual associado, então

$$
\mathbb{E}\left[g\left(\sigma\left(S^{\mathrm{o}}\right)\right)\right]=\mathbb{E}\left[g\left(S^{\mathrm{o}}\right)\right]
$$

para toda $g: \mathcal{N} \rightarrow \mathbb{R}$

Demonstração. Sem perda de generalidade considere $\lambda=1$ e observe que se $A=[-1 / 2,1 / 2]^{d}$, então 
pela Fórmula de Campbell (Proposição 2.14)

$$
\begin{aligned}
\mathbb{E}\left[g\left(\sigma\left(S^{\mathrm{o}}\right)\right)\right] & =\mathbb{E}\left[g\left(\tau_{\varphi\left(S^{\circ}\right)} S^{\mathrm{o}}\right)\right] \\
& =\mathbb{E}\left[\sum_{v \in S} \mathbf{1}_{A}(v) g\left(\tau_{\varphi\left(\tau_{v} S\right)+v} S\right)\right] \\
& =\mathbb{E}\left[\sum_{v \in S} \mathbf{1}_{A}(v) g\left(\tau_{\psi_{S}(v)} S\right)\right] \\
& =\mathbb{E}\left[\sum_{w \in S} \mathbf{1}_{A}\left(\psi_{S}^{-1}(w)\right) g\left(\tau_{w} S\right)\right] \\
& =\mathbb{E}\left[\sum_{w \in S} \mathbf{1}_{A}\left(\psi_{\tau_{w} S}^{-1}(0)+w\right) g\left(\tau_{w} S\right)\right] \\
& =\mathbb{E}\left[\int \mathbf{1}_{A}\left(\psi_{S^{\circ}}^{-1}(0)+x\right) g\left(S^{\mathrm{o}}\right) d x\right] \\
& =\mathbb{E}\left[g\left(S^{\mathrm{o}}\right)\right]
\end{aligned}
$$

Neste sentido segue a seguinte definição.

Definição 2.18. Diremos que um processo pontual $S^{\circ}$ é ponto estacionário se $\mathbb{P}\left(0 \in S^{\circ}\right)=1$ e a lei de $S^{\mathrm{o}}$ é invariante para todo shift bijetivo $\sigma$.

O exemplo a seguir foi dado por Häggström e citado em [27]

Exemplo 2.3. Diremos que $v, w$ em $\xi \in \mathcal{N}\left(\mathbb{R}^{d}\right)$ são vizinhos mais próximos mútuos em $\xi$ se o ponto de $\xi$ mais próximo de $v$ é $w$ e o ponto de $\xi$ mais próximo de $w$ é $v$. Defina então o shift pontual $\varphi: \mathcal{N}^{\circ} \rightarrow \mathbb{R}^{d}$ dado por

$$
\varphi(\xi)= \begin{cases}v, & \text { se } v \text { e } 0 \text { são vizinhos mais próximos mútuos em } \xi \\ 0, & \text { caso contrário. }\end{cases}
$$

Em [15] Heveling e Last mostram a família de shifts bijetivos é rica o suficiente para determinar as medidas de Palm. Ou seja, que ponto-estacionaridade é uma caracteristica apenas de medidas de Palm, no sentido de que todo processo $N$ ponto-estacionário é distribuído de acordo com a medida de Palm associada a algum processo pontual estacionário.

No mesmo artigo, os autores mostram também que existem mapeamentos pontuais bijetivos que não possuem pontos fixos, o que motiva uma outra pergunta: dado um processo $S^{\circ}$, é possível encontrar um mapeamento bijetivo $\varphi$ tal que $S^{\mathrm{o}}=\left\{\varphi^{n}\left(S^{\circ}\right) ; n \in \mathbb{Z}\right\}$, onde $\varphi^{n}\left(S^{\circ}\right)=\varphi^{n-1}\left(\tau_{\varphi\left(S^{\circ}\right)} S^{\circ}\right)=$ $\varphi^{n-1}\left(\sigma\left(S^{\mathrm{o}}\right)\right) ?$ 
Em [10] Ferrari et.al. resolveram este problema para processos de Poisson em dimensões 2 e 3 , construindo uma árvore invariante por isometrias de $\mathbb{R}^{d}$ utilizando todos os pontos do processo. Em [16] Holroyd e Peres estendem o resultado para processos de Poisson em todas as dimensões. Em 2004, Timar [28] mostrou que existe uma árvore com estas características para todo processo pontual ergódico com todos os grupos de isometrias triviais (em relação a $\mu$ ). Em [15] Heveling e Last conjeturaram que esta propriedade deve valer para todo processo estacionário não-periódico $S$, no sentido de que quase-certamente não existe $x \in \mathbb{R}^{d}$ tal que $\tau_{x} S=S$

Deste ponto em diante, trataremos apenas de processos pontuais para os quais possamos escrever $S^{\circ}=\left\{\varphi^{n}\left(S^{\circ}\right) ; n \in \mathbb{Z}\right\}$ para algum mapeamento pontual bijetivo $\varphi$. 


\section{Capítulo 3}

\section{O Passeio Aleatório e o Meio visto da Partícula}

Este capítulo será dedicado ao estudo do passeio aleatório simples no grafo de Delaunay. Os resultados obtidos aqui serão necessários para entendermos o processo de Harness, principal objeto de interesse neste trabalho, que construiremos no próximo capítulo. Para estudar o passeio aleatório utilizaremos sua relação com outro modelo: o meio visto da partícula. Este é o processo utilizado em [19] para o estudo do passeio aleatório no cluster infinito de percolação supercrítica.

Como comentado na introdução deste trabalho, apesar de enunciarmos os resultados principais em termos de um processo de Poisson em $\mathbb{R}^{d}$, os mesmos resultados valem para processos estacionários satisfazendo certas condições. Tais hipóteses são necessárias para que alguns resultados preliminares possam ser estendidos para outros processos pontuais estacionários. É interessante comentar que, mesmo que cada uma das condições listadas a seguir apareça na demostração de algum lema ou proposição, elas nunca aparecem todas ao mesmo tempo. De modo que é interessante que listemos todas estas hipóteses antes de seguirmos com nosso trabalho.

Hipóteses H1. Nas hipóteses que seguem $S$ é um processo pontual estacionário em $\mathbb{R}^{d}$ com intensidade finita, e versão de Palm $S^{\circ}$.

(i) $S$ é mixing;

(ii) $S$ é isotrópico, no sentido de sua lei ser invariante por isometrias de $\mathbb{R}^{d}$;

(iii) $S$ é aperiódico, i.e.,

$$
\mathbb{P}\left(\left\{\exists x \in \mathbb{R}^{d}: \tau_{x} S=S, x \neq 0\right\}\right)=0
$$

(iv) Nenhum conjunto de $d+2$ pontos em $S$ estão na fronteira de uma mesma bola;

(v) $\mathbb{E}\left[\left|V_{2}\left(0, S^{\mathrm{o}}\right)\right|^{3}\right]<\infty$, onde

$$
V_{2}\left(0, S^{\mathrm{o}}\right):=\bigcup_{v \in V(0)}|V(v)|
$$


(vi) $\mathbb{E}\left[\sum_{v \in S^{\circ}} a(0, v)\left|v_{1}\right|^{r}\right]<\infty$ para algum $r>4$;

(vii) $\mathbb{E}\left[\left|\ell\left(\mathrm{C}\left(0, S^{\mathrm{o}}\right)\right)\right|^{2}\right]<\infty$;

(viii) Podemos escrever $S^{\circ}=\left\{\varphi^{n}\left(S^{\circ}\right) ; n \in \mathbb{Z}\right\}$, para algum mapeamento pontual bijetivo $\varphi$.

A condição (iii), apesar de essencial na relação entre o passeio aleatório e o meio visto da partícula, pode ser facilmente contornada. Basta que associemos a cada ponto $v \in S$ uma marca $U_{v}$ uniforme em $[0,1]$, independentes entre si e independentes de $S$.

A hipótese (iv) torna possível a construção alternativa do grafo de Delaunay feita na observação 1.1.1 e, em última instância, tornam possíveis as estimativas feitas em (2.2), (2.3) e (2.4). A verficação de tal hipótese para o processo de Poisson homogêneo é parte dos resultados principais em [29].

As outras hipóteses foram verificadas para o processo de Poisson no capítulo anterior. Infelizmente, ainda não é claro que condições devem ser satisfeitas por um processo pontual para que tais hipóteses sejam verificadas.

Na próxima seção, vamos relembrar alguns resultados sobre processos Markovianos.

\subsection{Uma breve revisão sobre Processos de Markov}

Dado em e.m.c.s. $\mathcal{X}$, denote por $B F(\mathcal{X})$ o espaço das funções limitadas em $\mathcal{X}$. Considere agora um processo de Markov $\left\{S_{n} ; n \geq 0\right\}$ com valores em $\Xi$ e trajetórias $\mathbf{x}=\left(x_{n}\right)_{n \geq 0} \in \Xi^{\mathbb{Z}^{+}}$. Para $m \geq 0$ denote por $\theta_{m}$ e $R_{m}$, respectivamente, os operadores de translação temporal e reflexão temporal em $\Xi^{\mathbb{Z}^{+}}$, dados por

$$
\begin{aligned}
& \left(\theta_{m} \mathbf{x}\right)_{n}:=x_{n+m}, \\
& \left(R_{m} \mathbf{x}\right)_{n}:=x_{2 m-n}, \quad n \leq 2 m .
\end{aligned}
$$

Denotaremos por $P_{x}$ a lei de $\left\{S_{n} ; n \geq 0\right\}$ dado $S_{0}=x$. Do mesmo modo, denotaremos por $P_{\mu}$ a lei do processo quando a distribuição inicial é $\mu$, ou seja, $P_{\mu}=\int \mu(d x) P_{x}$. Diremos que $S_{t}$ é estacionário sob $\mu$ se, para toda $F \in B F\left(\Xi^{Z^{+}}\right)$cilíndrica (dependendo apenas de $\left(x_{n}\right)_{n \in I}$, para algum $I \subset \mathbb{Z}^{+}$ finito),

$$
E_{\mu}(F)=E_{\mu}\left(F \circ \theta_{m}\right), \quad \forall m \geq 0,
$$

o que, pela propriedade de Markov, é equivalente a

$$
E_{\mu}\left(f\left(S_{1}\right)\right)=E_{\mu}\left(f\left(S_{0}\right)\right), \quad \forall f \in B F(\Xi) .
$$


Da mesma forma, $S_{n}$ é reversível sob $\mu$ (ou $\mu$ reversível para $S_{n}$ ) se para toda $F \in B F\left(\Xi^{Z^{+}}\right.$) nas mesmas condições descritas acima,

$$
E_{\mu}(F)=E_{\mu}\left(F \circ R_{m}\right), \quad \forall m \geq 0,
$$

ou equivalentemente

$$
E_{\mu}\left(f\left(S_{0}\right) g\left(S_{1}\right)\right)=E_{\mu}\left(f\left(S_{1}\right) g\left(S_{0}\right)\right), \quad \forall f, g \in B F(\Xi)
$$

É importante observar que invariância é consequência direta de reversibilidade (basta fazer $f \equiv 1$ em (3.4)). Finalmente, diremos que um processo estacionário $S_{t}$ é ergódico (temporal) sob $\mu$, se $P_{\mu}(A) \in\{0,1\}$ sempre que $A \in \mathcal{B}\left(\Xi^{\mathbb{Z}^{+}}\right)$é invariante para $\theta_{1}$, no sentido de $\theta_{1} A=A$.

Lembramos também que, pelo teorema de extensão de Kolmogorov, a lei de todo processo estacionário com trajetória em $\Xi^{\mathbb{Z}^{+}}$pode ser estendida para $\Xi^{\mathbb{Z}}$. Neste caso consideraremos a extensão dos operadores (3.1) e (3.2) para sequências em $\Xi^{\mathbb{Z}}$.

\subsection{O Passeio Aleatório em $\mathcal{G}$ e o Meio Visto da Partícula}

Fixe uma configuração de pontos $\xi \in \mathcal{N}^{\circ}$, e considere $\tilde{X}_{n}$ um passeio aleatório simples em $\mathcal{G}(\xi)$. Para $v \in \xi$ denote por $\tilde{P}_{\xi}^{v}$ e $\tilde{E}_{\xi}^{v}$ a lei e esperança de $\tilde{X}_{n}$ em $(\xi)^{\mathbb{Z}^{+}}$com estado inicial $v$, ou seja, $\tilde{P}_{\xi}^{v}\left(\tilde{X}_{0}=v\right)=1 \mathrm{e}$

$$
\tilde{P}_{\xi}^{v}\left(\tilde{X}_{n}=v^{\prime} \mid \tilde{X}_{n-1}=w\right)=\frac{a\left(w, v^{\prime}, \xi\right)}{a(w, \xi)} .
$$

Para construir o passeio em tempo contínuo, seja $N=\left\{T_{v} ; v \in \mathbb{N}\right\}$ um processo de Poisson homogêneo de taxa 1 em $\mathbb{R}^{+}$, independente de $\left(\tilde{X}_{n}\right)_{n \geq 0}$. Defina agora

$$
X_{t}:=\tilde{X}_{N(t)}
$$

onde $N(t)=N(0, t]$ é o número de pontos de $N$ no intervalo $(0, t]$. Faça $P_{\xi}^{v}=\tilde{P}_{\xi}^{v} \otimes Q$, onde $Q$ é a lei de $N$ em $\left(\mathcal{N}\left(\mathbb{R}^{+}\right), \mathcal{B}\left(\mathcal{N}\left(\mathbb{R}^{+}\right)\right)\right)$.

Para tentar compreender melhor algumas propriedades dos passeios $X_{t}$ e $\tilde{X}_{n}$ com estado inicial $0 \in \xi$, defina o processo

$$
S_{n}=\tau_{\tilde{X}_{n}} \xi
$$

Tal processo, pode ser interpretado como o meio vista da partícula que realiza o passeio aleatório $\tilde{X}_{n}$ com lei $\tilde{P}_{\xi}^{0}$. $S_{n}$ é claramente um processo de Markov com valores em $\mathcal{N}^{\circ}$ e, assim como anteriormente, denotaremos por $P_{\xi}$ a lei de $S_{n}$ em $\left(\mathcal{N}^{\circ}\right)^{\mathbb{Z}^{+}}$com estado inicial $\xi$. 
Tendo em mãos a trajetória de $S_{n}$, vamos agora tentar recuperar a trajetória do passeio $\tilde{X}_{n}$. Para isto, no entanto, é necessário que o meio onde acontece o passeio seja aperiódico. Relembrando, diremos que um processo pontual $S$ é aperiódico $\operatorname{se} \mathbb{P}\left(S \in \mathcal{M}^{\prime}\right)=1$, onde $\mathcal{M}^{\prime} \subseteq \mathcal{N}$ é dado por

$$
\mathcal{M}^{\prime}=\left\{\xi \in \mathcal{N}: \tau_{x} \xi \neq \xi \text { para todo } x \in \mathbb{R}^{d}, x \neq 0\right\}
$$

Para o que segue, considere portanto um processo $S$ aperiódico.

Seja $\Lambda: \mathcal{M}^{\prime} \times \mathcal{N}^{\circ} \rightarrow \mathbb{R}^{d}$ dada por

$$
\Lambda\left(\xi, \xi^{\prime}\right)= \begin{cases}v ; & \text { se } \xi^{\prime}=\tau_{v} \xi \\ 0 ; & \text { caso contrário. }\end{cases}
$$

Note que, se $S_{0}=\xi \in \mathcal{M}^{\prime}$, então $S_{n} \in \mathcal{M}$ e

$$
X_{n}-X_{n-1}=\Lambda\left(S_{n-1}, S_{n}\right)
$$

para todo $n \geq 0$. Mais do que isso, vale também que

$$
X_{n}-X_{0}=\Lambda\left(S_{0}, S_{n}\right)=\sum_{k=1}^{n} \Lambda\left(S_{k-1}, S_{k}\right) .
$$

Dado um processo pontual ergódico $S^{\mathrm{o}}$ em $\mathbb{R}^{d}$, denote por $\nu_{o}$ a medida de probabilidade em $\left(\mathcal{N}^{\circ}, \mathcal{B}\left(\mathcal{N}^{\circ}\right)\right)$ dada por

$$
\int \phi(\xi) \nu_{o}(d \xi)=\frac{1}{\mathbb{E}[|V(0)|]} \mathbb{E}\left[|V(0)| \phi\left(S^{\mathrm{o}}\right)\right]
$$

Lema 3.1. O processo $\left(S_{n}\right)_{n \geq 0}$ é reversível e ergódico sob $\nu_{o}$.

Demonstração. Verificar reversibilidade é equivalente a mostrar que

$$
\int E_{\xi}\left[f(\xi) g\left(S_{1}\right)\right] \nu_{o}(d \xi)=\int E_{\xi}\left[f\left(S_{1}\right) g(\xi)\right] \nu_{o}(d \xi)
$$

para $f, g$ limitadas.

Para isso, faça $\phi\left(v, w, S^{\mathrm{o}}\right)=a\left(v, w, S^{\mathrm{o}}\right) f\left(\tau_{v} S^{\mathrm{o}}\right) g\left(\tau_{w} S^{\mathrm{o}}\right)$ e note que $\phi$ é covariante e integrável, e 
portanto, pelo principio de transporte de massa, vale que

$$
\begin{aligned}
\int E_{\xi}\left[f(\xi) g\left(S_{1}\right)\right] \nu_{o}(d \xi) & =(1 / \mathbb{E}[|V(0)|]) \mathbb{E}\left[\sum_{v \in S^{\circ}} a\left(0, v, S^{\mathrm{o}}\right) f\left(S^{\mathrm{o}}\right) g\left(\tau_{v} S^{\mathrm{o}}\right)\right] \\
& =(1 / \mathbb{E}[|V(0)|]) \mathbb{E}\left[\sum_{v \in S^{\mathrm{o}}} \phi\left(0, v, S^{\mathrm{o}}\right)\right] \\
& =(1 / \mathbb{E}[|V(0)|]) \mathbb{E}\left[\sum_{v \in S^{\mathrm{o}}} \phi\left(v, 0, S^{\mathrm{o}}\right)\right] \\
& =(1 / \mathbb{E}[|V(0)|]) \mathbb{E}\left[\sum_{v \in S^{\mathrm{o}}} a\left(0, v, S^{\mathrm{o}}\right) f\left(\tau_{v} S^{\mathrm{o}}\right) g\left(S^{\mathrm{o}}\right)\right] \\
& =\int E_{\xi}\left[f\left(S_{1}\right) g(\xi)\right] \nu_{o}(d \xi) .
\end{aligned}
$$

Para mostrar a ergodicidade, considere um evento $A \in \mathcal{B}\left(\mathcal{N}^{\circ}\right)$ tal que $\theta_{1} A=A$, e note que se $\xi \in A$ então $\tau_{v} \xi \in A$ para todo $v \in \xi$. De fato, pela conectividade de $\mathcal{G}(\xi)$, dado $v \in \xi$ existe $n>0$ tal que $\tau_{v} \xi \in \theta_{n} A=A$. Segue portanto de Proposição 2.9 que $\mu_{o}(A):=\mathbb{P}\left(S^{\circ} \in A\right) \in\{0,1\}$ e, como $\nu_{o} \ll \mu_{o}$ e $\mu_{o} \ll \nu_{o}$, segue que $\nu_{o}(A) \in\{0,1\}$.

O Lema 3.1 nos permite agora compreender melhor algumas propriedades do passeio aleatório $X_{t}$.

Proposição 3.2. Dados $r \geq 1$ e $c \in \mathbb{R}^{d}$, se $S^{o}$ é tal que $\mathbb{E}\left[\sum_{v \in V(0)}|(c \cdot v)|^{r}\right]<\infty$ então existe uma constante $C>0$ tal que

$$
\mathbb{E}\left(E_{S^{\circ}}^{0}\left[\left|\left(c \cdot X_{t}\right)\right|^{r}\right]\right) \leq C M_{r}(t)
$$

onde $M_{r}(t)$ é o r-ésimo momento de uma variável aleatório de Poisson com parâmetro $t$.

Demonstração. Note que

$$
\mathbb{E}\left(E_{S^{\circ}}^{0}\left[\left|\left(c \cdot X_{t}\right)\right|^{r}\right]\right)=\sum_{n=0}^{\infty} \mathbb{E}\left(E_{S^{\circ}}^{0}\left[\left|\left(c \cdot \tilde{X}_{n}\right)\right|^{r} \mathbf{1}_{\{N(t)=n\}}\right]\right)=\sum_{n=0}^{\infty} \mathbb{E}\left(E_{S^{\circ}}^{0}\left[\left|\left(c \cdot \tilde{X}_{n}\right)\right|^{r}\right]\right) \mathrm{e}^{-t} \frac{t^{n}}{n !} .
$$

Observe que $\left(c \cdot \tilde{X}_{n}\right)=\sum_{k=1}^{n}\left(c \cdot\left[\tilde{X}_{k}-\tilde{X}_{k-1}\right]\right)$,

e portanto, da desigualdade de Hölder, segue que

$$
\left|\left(c \cdot \tilde{X}_{n}\right)\right|^{r} \leq n^{r-1} \sum_{k=1}^{n}\left|\left(c \cdot\left[\tilde{X}_{k}-\tilde{X}_{k-1}\right]\right)\right|^{r},
$$


Da reversibilidade de $\nu_{o}$ concluímos então que

$$
\begin{aligned}
\mathbb{E}\left[E_{S^{\circ}}^{0}\left[\left|\left(c \cdot \tilde{X}_{n}\right)\right|^{r}\right]\right. & \leq n^{r-1} \sum_{k=1}^{n} \mathbb{E}\left(E_{S^{\circ}}^{0}\left[\left|\left(c \cdot\left[\tilde{X}_{k}-\tilde{X}_{k-1}\right]\right)\right|^{r}\right]\right) \\
& \leq n^{r-1} \mathbb{E}[|V(0)|] \sum_{k=1}^{n} E_{\nu_{o}}\left[\left|\left(c \cdot \Lambda\left(S_{k-1}, S_{k}\right)\right)\right|^{r}\right] \\
& =n^{r} \mathbb{E}[|V(0)|] E_{\nu_{o}}\left[\left|\left(c \cdot \Lambda\left(S_{0}, S_{1}\right)\right)\right|^{r}\right] \\
& =n^{r} \mathbb{E}\left[|V(0)| E_{S^{\circ}}^{0}\left[\left|\left(c \cdot \tilde{X}_{1}\right)\right|^{r}\right]\right] \\
& =n^{r} \mathbb{E}\left[\sum_{v \in S^{\circ}} a\left(0, v, S^{\mathrm{o}}\right)|(c \cdot v)|^{r}\right],
\end{aligned}
$$

e portanto, fazendo $C:=\mathbb{E}\left[\sum_{v \in S^{\circ}} a\left(0, v, S^{\circ}\right)|(c \cdot v)|^{r}\right]$, obtemos

$$
\mathbb{E}\left(E_{S^{\circ}}^{0}\left[\left|\left(c \cdot X_{t}\right)\right|^{r}\right]\right) \leq C \sum_{n=0}^{\infty} n^{r} \mathrm{e}^{-t} \frac{t^{n}}{n !}<C M_{r}(t) .
$$

O Lema abaixo é parte integrante da demonstração do Teorema 2.1 de [19]. Incluiremos uma versão resumida da demonstração original apenas para completude do trabalho.

Lema 3.3. Se $S^{\text {o }}$ é tal que

$$
\mathbb{E}\left[\sum_{v \in V\left(0, S^{\circ}\right)}|c \cdot v|^{2}\right]<\infty
$$

então

$$
\lim _{n \rightarrow \infty} \frac{\mathbb{E}\left(|V(0)| E_{S^{\circ}}^{0}\left[\left|\left(c \cdot \tilde{X}_{n}\right)\right|^{2}\right]\right)}{n}<\infty
$$

$e$

$$
\lim _{t \rightarrow \infty} \frac{\mathbb{E}\left(|V(0)| E_{S^{\circ}}^{0}\left[\left|\left(c \cdot X_{t}\right)\right|^{2}\right]\right)}{n}<\infty
$$

Demonstração. Seguindo a equação (3.8) defina

$$
Y_{n}=\left(c \cdot \Lambda\left(S_{n-1}, S_{n}\right)\right),
$$

e note que $\left(c \cdot \tilde{X}_{n}\right)=\sum_{k=1}^{n} Y_{k}$, e portanto

$$
\frac{1}{\mathbb{E}[|V(0)|]} \mathbb{E}\left(|V(0)| E_{S^{\circ}}^{0}\left[\left|\left(c \cdot \tilde{X}_{n}\right)\right|^{2}\right]\right)=E_{\nu_{o}}\left[\left(\sum_{k=1}^{n} Y_{k}\right)^{2}\right] \text {. }
$$


Seja $\mathcal{F}_{n}$ a $\sigma$-álgebra gerada por $\left\{S_{k} ; k=0, \ldots, n\right\}$, e defina

$$
\phi_{n-1}=E_{\nu_{o}}\left[Y_{n} \mid \mathcal{F}_{n-1}\right] .
$$

Note agora que a sequência

$$
M_{n}=Y_{n}-\phi_{n-1}
$$

é estacionária ( $\operatorname{sob} \nu_{o}$ ), quadrado-integrável (segue do Proposição 3.2) e tal que

$$
\sum_{k=1}^{n} M_{k}
$$

é um martingal com relação a $\left\{\mathcal{F}_{k} ; k \geq 0\right\}$.

Observe também que

$$
E_{\nu_{o}}\left[\left(\sum_{k=1}^{n} Y_{k}\right)\left(\sum_{k=0}^{n} \phi_{k}\right)\right]=0 .
$$

De fato, escrevendo $\phi\left(S_{0}, \ldots, S_{n}\right)=\sum_{k=0}^{n} \phi_{k}$, segue da reversibilidade de $\nu_{o}$ que

$$
\begin{aligned}
E_{\nu_{o}}\left[\left(\sum_{k=1}^{n} Y_{k}\right)\left(\sum_{k=0}^{n} \phi_{k}\right)\right] & =E_{\nu_{o}}\left[\left(\sum_{k=1}^{n} \Lambda\left(S_{k-1}, S_{k}\right)\right) \phi\left(S_{0}, \ldots, S_{n}\right)\right] \\
& =E_{\nu_{o}}\left[\left(\sum_{k=1}^{n} \Lambda\left(S_{k}, S_{k-1}\right)\right) \phi\left(S_{n}, \ldots, S_{0}\right)\right] \\
& =-E_{\nu_{o}}\left[\left(\sum_{k=1}^{n} \Lambda\left(S_{k-1}, S_{k}\right)\right) \phi\left(S_{0}, \ldots, S_{n}\right)\right],
\end{aligned}
$$

e (3.11) segue.

Concluímos então que

$$
\begin{aligned}
n E_{\nu_{o}}\left[M_{1}^{2}\right] & =E_{\nu_{o}}\left[\left(\sum_{k=1}^{n} Y_{k}\right)^{2}\right]+E_{\nu_{o}}\left[\left(\sum_{k=0}^{n-1} \phi_{k}\right)^{2}\right]-2 E_{\nu_{o}}\left[\left(\sum_{k=1}^{n} Y_{k}\right)\left(\sum_{k=0}^{n-1} \phi_{k}\right)\right] \\
& =E_{\nu_{o}}\left[\left(\sum_{k=1}^{n} Y_{k}\right)^{2}\right]+E_{\nu_{o}}\left[\left(\sum_{k=0}^{n-1} \phi_{k}\right)^{2}\right]+2 E_{\nu_{o}}\left[\phi_{n}\left(\sum_{k=1}^{n} Y_{k}\right)\right] .
\end{aligned}
$$

Usando a desigualdade de Cauchy-Schwarz no terceiro termo da última soma, concluímos que os dois primeiros termos são de ordem $n$ e o terceiro é da ordem de $n^{\frac{1}{2}}$, e (3.9) segue. Usando agora que $X_{t}=\tilde{X}_{N(t)}$ e $N(t)$ é um Poisson homogêneo, concluimos (3.10). 
Observação 3.2.1. Na demonstração do Teorema 2.1 em [19], os autores são mais exatos no resultado, conseguindo calcular inclusive o valor de

$$
\lim _{n \rightarrow \infty} \frac{1}{n} E_{\nu_{o}}\left[\left(\sum_{k=0}^{n-1} \phi_{k}\right)^{2}\right]
$$

mas não precisamos destes detalhes para nosso propósito. 


\section{Capítulo 4}

\section{O Processo de Harness}

A partir deste ponto, e pelo resto do trabalho, $S$ denotará um processo de Poisson homogêneo em $\mathbb{R}^{d}$, e $S^{\mathrm{o}}=S+\delta_{0}$ sua versão de Palm. Para facilitar a leitura, e sem perda de generalidade, consideraremos que $S^{\circ}$ tem intensidade $\lambda=1$.

\subsection{O Processo de Harness sem Ruído}

Dada uma configuração de pontos $\xi \in \mathcal{N}$, o Processo de Harness $\eta_{t}^{\gamma} \in \mathbb{R}^{\xi}$ consiste em, iniciando de uma configuração $\gamma \in \mathbb{R}^{\xi}$, atualizar o valor de $\eta_{t}^{\gamma}$ em cada ponto $v \in \xi$, à taxa 1 , para a média aritmética da altura de seus vizinhos em $\mathcal{G}(\xi)$, isto é, um processo $\eta_{t}^{\gamma}$ com valores em $\mathbb{R}^{\xi}, \eta_{0}^{\gamma}=\gamma \in \mathbb{R}^{\xi}$ e gerador

$$
L^{\xi} g(\eta)=\sum_{v \in \xi}\left[g\left(M_{v}^{\xi} \eta\right)-g(\eta)\right]
$$

agindo em funções $g: \mathbb{R}^{\xi} \rightarrow \mathbb{R}$ limitadas, dependendo apenas de uma quantidade finita de pontos em $\xi$, e onde

$$
M_{v}^{\xi} \eta(w)= \begin{cases}\frac{1}{|V(v, \xi)|} \sum_{u \in \xi} a(v, u, \xi) \eta(u) & \text { se } w=v \\ \eta(w) & \text { se } w \neq v\end{cases}
$$

Para nossos objetivos, será suficiente considerarmos como configuração inicial apenas funções lineares, ou seja, $\gamma(v)=\pi_{c}(v)=(c \cdot v)$ para algum $c \in \mathbb{R}^{d}$. Deste ponto em diante, fixado $c \in \mathbb{R}^{d}$ notaremos $\eta_{t}^{c}:=\eta_{t}^{\pi_{c}}$.

\subsubsection{A Construção Gráfica}

Para construção gráfica do processo, fixado $\xi \in \mathcal{N}$, vamos associar a cada ponto $v \in \xi$ um processo de Poisson $\mathcal{T}(v)=\left\{T_{1}(v) ; T_{2}(v) ; \ldots\right\}$ de taxa 1 em $\mathbb{R}^{+}$, e um conjunto $\mathcal{U}(v)=\left\{U_{1}(v) ; U_{2}(v) ; \ldots\right\}$ de variáveis aleatórias independentes e uniformemente distribuídas no intervalo $[0,1]$. Em outras 
palavras, consideraremos configurações de pontos $\bar{\xi} \in \mathcal{N}^{m}\left(\mathbb{R}^{d} \times \mathcal{N}\left(\mathbb{R}^{+}\right) \times[0,1]\right)$, com configuração de base $\xi \in \mathcal{N}\left(\mathbb{R}^{d}\right)$.

Fixe $t>0$ e considere uma família $\left\{B_{[t, s]}^{v} ; s \leq t, v \in \xi\right\}$ de passeios aleatórios simples nos pontos de $\xi$, para trás no tempo, começando em $v \in \xi$ no instante $t$, andando de acordo com as marcas temporais de $\mathcal{T}=\{\mathcal{T}(v) ; v \in \xi\}$ e d as variáveis de $\mathcal{U}=\{\mathcal{U}(v) ; v \in \xi\}$. Ou seja, $B_{[t, t]}^{v}=v$ e se no instante $s+$ o passeio está em $w \in \xi$ e $T_{n}(w)=s$ então o passeio utiliza a variável $U_{n}(w)$ para escolher uniformemente um dos vizinhos de $w$ (cada vizinho é escolhido com probabilidade $\frac{1}{|V(w, \xi)|}$ ). Denote por $P_{\xi}$ a lei conjunta de $\left(B_{[t, s]}^{v} ; v \in \xi\right)$.

É interessante observar que a lei de $B_{[t, 0]}^{v}$ sob $P_{\xi}$ é a mesma de $X_{t}$ sob $P_{\xi}^{v}$, para todo $v \in \xi$, ou seja,

$$
\left.E_{\xi}\left[f\left(B_{[t, 0]}^{v}\right)\right]\right]=E_{\xi}^{v}\left[f\left(X_{t}\right)\right]
$$

Defina $\eta_{t}^{\gamma}(v)$ como a média de $\gamma\left(B_{[t, 0]}^{v}\right)$ condicionada aos tempos de salto $\mathcal{T}$. Ou seja, abusando da notação e chamando de $\mathcal{T}$ a $\sigma$-álgebra gerada por $\mathcal{T}$ e definindo

$$
p_{t}(v, w, \bar{\xi})=E_{\xi}\left[\mathbf{1}_{\left\{B_{[t, 0]}^{v}=w\right\}} \mid \mathcal{T}\right]
$$

então

$$
\eta_{t}^{\gamma}(v)=\sum_{w \in \xi} p_{t}(v, w, \bar{\xi}) \gamma(w)
$$

Observe que, do modo como foi definido, o processo não depende das marcas de $\mathcal{U}$. Por esta razão, deste ponto em diante podemos esquecer de tais marcas e considerar $\bar{\xi}$ como um elemento de $\mathcal{N}^{m}\left(\mathbb{R}^{d} \times \mathcal{N}\left(\mathbb{R}^{+}\right)\right)$.

Olhando este processo para frente no tempo, verificamos que $\eta_{0}^{\gamma}(v)=\gamma(v)$ e, se um relógio $T_{n}(v)$ toca em um sítio $v \in \xi$ em um instante $t$, então,

$$
p_{t}(v, w, \bar{\xi})=\sum_{u \in \xi} \frac{a(v, u, \xi)}{V(v)} p_{t-}(u, w, \bar{\xi})
$$

e portanto a altura em $v$ é atualizada para

$$
\eta_{t}^{\gamma}(v)=\sum_{w \in \xi} \sum_{u \in \xi} \frac{a(v, u, \xi)}{V(v)} p_{t-}(u, w, \bar{\xi}) \gamma(w)=M_{v}^{\xi} \eta_{t-}^{\gamma}(v)
$$

enquanto as alturas nos outros pontos de $\xi$ permanecem inalteradas.

Desde ponto em diante, sempre que necessário, tornaremos a dependência de $\eta_{t}^{\gamma}$ em $\bar{\xi}$ explicita, 
escrevendo

$$
\eta_{t}^{\gamma}(v)=\eta_{t}^{\gamma}(v, \bar{\xi})
$$

É interessante notar que

$$
p_{t}(v, w, \bar{\xi})=p_{t}\left(0, w-v, \tau_{v} \bar{\xi}\right)
$$

e portanto, dado $c \in \mathbb{R}^{d}$ vale que

$$
\begin{aligned}
\eta_{t}^{c}(v, \bar{\xi}) & =\sum_{w \in \bar{\xi}} p_{t}(v, w, \bar{\xi})(c \cdot w) \\
& =\sum_{w \in \bar{\xi}} p_{t}\left(0, w-v, \tau_{u} \bar{\xi}\right)(c \cdot[w-v])+(c \cdot v) \\
& =\sum_{w \in \tau_{v} \bar{\xi}} p_{t}\left(0, w, \tau_{v} \bar{\xi}\right)(c \cdot w)+(c \cdot v) \\
& =\eta_{t}^{c}\left(0, \tau_{v} \bar{\xi}\right)+(c \cdot v)
\end{aligned}
$$

Proposição 4.1. Dado $c \in \mathbb{R}^{d}$, o processo $\eta_{t}^{\gamma}$, com $\gamma(v)=(c \cdot v)$, está bem definido e tem gerador (4.1).

Demonstração. Para verificar que o processo está bem definido, basta mostrar que a soma do lado direito de (4.4) é finita.

Para isso, denote por $\bar{\mu}_{\mathrm{o}}$ a lei do processo pontual marcado $\bar{S}^{\mathrm{o}}$ com processo base $S^{\mathrm{o}}$ e marcas dadas por $\left\{\mathcal{T}(v) ; v \in S^{\circ}\right\}$, e observe que, por (4.6), só precisamos verificar que, para todo $v \in S^{\circ}$

$$
\sum_{w \in \tau_{v} \bar{S}^{\circ}} p_{t}\left(0, w, \tau_{v} \bar{S}^{\mathrm{o}}\right)(c \cdot w)<\infty, \quad \bar{\mu}_{\mathrm{o}} \text {-quase certamente. }
$$

Como $S^{\text {o }}$ é ponto-estacionário, precisamos verificar (4.7) apenas para $v=0$. Mas, de (4.3) e pela Proposição 3.2

$$
\int\left|\sum_{w \in \bar{\xi}} p_{t}(0, w, \bar{\xi})(c \cdot w)\right| \bar{\mu}_{\mathrm{o}}(d \bar{\xi}) \leq \mathbb{E}\left[E_{S^{\circ}}\left[\left|\left(c \cdot B_{[t, 0]}^{0}\right)\right|\right]\right]=\mathbb{E}\left[E_{S^{\circ}}^{0}\left|\left(c \cdot X_{t}\right)\right|\right]<\infty
$$

e a afirmativa segue.

A demostração de que $\eta_{t}^{\gamma}$ tem gerador dado por (4.1), segue de (4.5) e de $\xi$ ser localmente finito, utilizando passos usuais (ver, por exemplo, [13]). 


\subsection{Funções harmônicas em $\mathcal{G}$}

Nesta seção vamos fixar notações e conceitos sobre funções definidas nos vértices e elos do grafo aleatório $\mathcal{G}\left(S^{\circ}\right)$. Como já foi comentado na seção anterior, as funções consideradas neste trabalho carregam naturalmente uma dependência do processo marcado $\bar{S}^{\circ}$, por esta razão consideraremos funções tomando valores nos espaços

$$
\Xi_{1}=\left\{(x, \bar{\xi}) \in \mathbb{R}^{d} \times \mathcal{N}^{m}: x \in \xi\right\}
$$

$\mathrm{e}$

$$
\Xi_{2}=\left\{(x, y, \bar{\xi}) \in \mathbb{R}^{d} \times \mathbb{R}^{d} \times \mathcal{N}^{m}: x, y \in \xi\right\}
$$

Do modo como foi definido o grafo $\mathcal{G}\left(S^{\circ}\right)$ é não-orientado, mas por razões de coerência, precisaremos distinguir elos de acordo com sua orientação. Por esta razão consideraremos $\mathcal{G}\left(S^{\mathrm{o}}\right)$ orientado, mas tal que se $(x, y) \in \mathcal{E}\left(S^{\circ}\right)$, então $(y, x) \in \mathcal{E}\left(S^{\circ}\right)$.

Agora, dada uma função $f: \Xi_{1} \rightarrow \mathbb{R}$, defina o gradiente de $f$ como a função $\nabla f: \Xi_{2} \rightarrow \mathbb{R}$ dada por

$$
\nabla f\left(v, w, \bar{S}^{\mathrm{o}}\right)=f\left(w, \bar{S}^{\mathrm{o}}\right)-f\left(v, \bar{S}^{\mathrm{o}}\right)
$$

Deste ponto em diante, denotaremos por campos todas funções definidas em $\Xi_{2}$. Diremos que um campo $\zeta$ é um fluxo em $\mathcal{G}$ se

$$
\zeta\left(v, w, \bar{S}^{\mathrm{o}}\right)=-\zeta\left(w, v, \bar{S}^{\mathrm{o}}\right)
$$

para qualquer $(v, w) \in \mathcal{E}\left(S^{\mathrm{o}}\right)$.

Diremos que um campo $\zeta$ é covariante se para todo $v, w \in \bar{\xi}$

$$
\zeta(v, w, \bar{\xi})=\zeta\left(0, w-v, \tau_{v} \bar{\xi}\right)
$$

Note que, por (4.6),

$$
\nabla \eta_{t}^{\gamma}\left(v, w, \bar{S}^{\mathrm{o}}\right)=\eta_{t}^{\gamma}\left(w-v, \tau_{v} \bar{S}^{\mathrm{o}}\right)+(c \cdot v)-\eta_{t}^{\gamma}\left(0, \tau_{v} \bar{S}^{\mathrm{o}}\right)-(c \cdot v)=\nabla \eta_{t}^{\gamma}\left(0, w-v, \tau_{v} \bar{S}^{\mathrm{o}}\right),
$$

para todo instante de tempo $t \geq 0$, e portanto $\nabla \eta_{t}^{\gamma}$ é um campo covariante.

Mais do que isso, se definirmos $\phi_{t}\left(v, S^{\circ}\right)=\eta_{t}^{\gamma}\left(0, \tau_{v} \bar{S}^{\circ}\right)$, então

$$
\eta_{t}^{\gamma}\left(v, \bar{S}^{\mathrm{o}}\right)=\gamma(v)+\phi_{t}\left(v, \bar{S}^{\mathrm{o}}\right)
$$


e $\phi_{t}$ é tal que $\phi_{t}\left(v, \bar{S}^{\mathrm{o}}\right)=\phi_{t}\left(0, \tau_{v} \bar{S}^{\mathrm{o}}\right)$.

Dado um fluxo $\zeta$ em $\mathcal{G}$ definimos o divergente de $\zeta$ como a função $\operatorname{div} \zeta: \Xi_{1} \rightarrow \mathbb{R}$ dada por

$$
(\operatorname{div} \zeta)\left(v, \bar{S}^{\mathrm{o}}\right)=\sum_{w \in V\left(v, S^{\circ}\right)} \zeta\left(v, w, \bar{S}^{\mathrm{o}}\right)
$$

Continuando, para qualquer função $f: \Xi_{1} \rightarrow \mathbb{R}$ definimos o laplaciano discreto de $f$ como a função $\Delta f: \Xi_{1} \rightarrow \mathbb{R}$ dada por

$$
\Delta f(v)=(\operatorname{div} \nabla f)(v)=\sum_{w \in V\left(v, \bar{S}^{\circ}\right)} \nabla f\left(v, w, \bar{S}^{\circ}\right)=\sum_{w \in V\left(v, \bar{S}^{\circ}\right)}(f(w)-f(v)) .
$$

Diremos que uma função $h: \Xi_{1} \rightarrow \mathbb{R}$ é harmônica em $\mathcal{G}$ se, para quase toda realização de $\bar{S}^{\circ}$,

$$
\Delta h\left(v, S^{\mathrm{o}}\right)=0, \quad \text { para todo } v \in S^{\mathrm{o}} .
$$

Segue diretamente desta definição que $h$ é harmônica se e somente se o valor da função $h$ em um vértice é a média aritmética do valor da função nos vizinhos deste vértice, ou seja,

$$
h\left(v, \bar{S}^{\mathrm{o}}\right)=\frac{1}{\left|V\left(v, S^{\mathrm{o}}\right)\right|} \sum_{w \in V\left(v, \bar{S}^{\circ}\right)} h\left(w, \bar{S}^{\mathrm{o}}\right), \quad \text { para todo } v \in S^{\mathrm{o}} .
$$

\section{3 $\mathrm{O}$ espaço $\mathcal{H}$}

Como primeiro passo da solução do nosso problema, vamos caracterizar o gradiente $\nabla \eta_{t}^{c}$ como elemento de um espaço de Hilbert.

Com este fim, considere o processo pontual marcado $\bar{S}^{\text {o }}$, defina $\mathcal{X}=\mathbb{R}^{d} \times \mathbb{R}^{d} \times \mathcal{N}^{m}$ e denote por $\mathcal{C}$ a medida em $(\mathcal{X}, \mathcal{B}(\mathcal{X}))$ tal que

$$
\int_{\mathcal{X}} \zeta d \mathcal{C}:=\frac{1}{2} \mathbb{E}\left[\sum_{v \in V\left(0, S^{\circ}\right)} \zeta\left(0, v, \bar{S}^{\circ}\right)\right] .
$$

A existência de tal medida é consequência das considerações feitas no capítulo 13 de [7]. Fazemos um resumo de tais resultados no apêndice deste trabalho. Deste ponto em diante denotaremos

$$
\mathcal{C}(\zeta):=\int_{\mathcal{X}} \zeta d \mathcal{C}
$$


Denote por $\mathcal{H}$ o espaço $L_{2}(\mathcal{X}, \mathcal{C})$, e note que se $\zeta_{1}$ e $\zeta_{2}$ são dois campos em $\mathcal{H}$ tais que $\zeta_{1}\left(0, v, \bar{S}^{\mathrm{o}}\right)=$ $\zeta_{2}\left(0, v, \bar{S}^{\mathrm{o}}\right)$ para todo $v \in V\left(0, S^{\mathrm{o}}\right)$ então $\zeta_{1}=\zeta_{2}$ em $\mathcal{H}$, ou seja, para determinar um campo em $\mathcal{H}$ basta olharmos para seus valores nos elos saindo da origem.

Nossa motivação vem da teoria relacionada a um caso distinto do nosso: funções harmônicas em redes infinitas, mas com energia finita. Vamos esclarecer. Considere um grafo genérico e conexo $\mathcal{G}=(\mathcal{V}, \mathcal{E})$. Considere (como temos feito desde o início) que $\mathcal{E}$ é orientado, e que $e \in \mathcal{E}$ se e só se $-e \in \mathcal{E}$. Tome então um fluxo $\zeta$ em $\mathcal{E}$ e defina a energia de $\zeta$ como o funcional

$$
\mathbf{E}[\zeta]=\frac{1}{2} \sum_{e \in E}|\zeta(e)|^{2}
$$

Tal funcional é de central importância na teoria de funções harmônicas. Pode-se mostrar, por exemplo, que gradientes de funções harmônicas são minimizantes locais $\mathbf{E}[\cdot]$ (ver Apêndice). Outra observação importante é que a família dos fluxos em $\mathcal{E}$, com energia finita, formam um espaço de Hilbert, e é neste ponto que queremos focar nossa atenção.

Apesar de importante, este resultado claramente não se aplica em nosso contexto. Observe porém que, ao considerarmos campos covariantes, uma nova possíbilidade se abre. Para entender o que temos em mente, fixada uma configuração de pontos $\xi$ e uma caixa limitada $A$, denote por $\mathcal{E}_{A}(\xi)$ todos os elos $(v, w) \in \mathcal{E}(\xi)$ tais que $v \in \xi \cap A$. Segue então que, para um campo covariante $\zeta$,

$$
\sum_{e \in \mathcal{E}_{A}(\xi)} \zeta(e)=\sum_{v \in \xi \cap A} \sum_{w \in V(v, \xi)} \zeta(v, w, \xi)=\sum_{v \in \xi \cap A} \sum_{w \in V\left(0, \tau_{v} \xi\right)} \zeta\left(0, w, \tau_{v} \xi\right) .
$$

Deste modo, fazendo com a a caixa $A$ cresça para $\mathbb{R}^{d}$, segue do Teorema Ergódico Pontual (ver Teorema 2.15) que

$$
\lim _{A \rightarrow \mathbb{R}^{d}} \frac{1}{2 \ell(A)} \sum_{v \in S \cap A} \sum_{w \in V(v)} \zeta\left(v, w, \bar{S}^{\mathrm{o}}\right)=\mathcal{C}(\zeta), \quad \text { q.c.. }
$$

Esta relação permite que interpretemos $\mathcal{C}\left(|\zeta|^{2}\right)$ como uma espécie de "energia média" associada ao fluxo $\zeta$. Outras analogias podem ser feitas para reforçar esta interpretação, mas, por ora, nos satisfaremos com esta. Ao longo do resto do trabalho tentaremos manter esta relação em mente, o que será útil para que façamos novas interpretações de resultados, a primeira vista, apenas técnicos.

Continuando com a caracterização de $\mathcal{H}$, vamos agora enunciar uma fórmula de integração por partes, associando campos em $\mathcal{H}$ com funções definidas em $\Xi_{1}$.

Proposição 4.2 (Fórmula de Integração por Partes). Seja $f: \Xi_{1} \rightarrow \mathbb{R}$ tal que

$$
f\left(v, \bar{S}^{\mathrm{o}}\right)=f\left(0, \tau_{v} \bar{S}^{\mathrm{o}}\right)
$$


$e \zeta: \Xi_{2} \rightarrow \mathbb{R}$ um fluxo covariante. Nestas condições, se $\nabla f, \zeta \in \mathcal{H}$ então

$$
\mathcal{C}(\nabla f \cdot \zeta)=-\mathbb{E}[f \cdot \operatorname{div} \zeta]:=-\mathbb{E}\left[f\left(0, \bar{S}^{\mathrm{o}}\right) \operatorname{div} \zeta\left(0, \bar{S}^{\mathrm{o}}\right)\right]
$$

Demonstração. Note que

$$
\begin{aligned}
\mathcal{C}(\nabla f \cdot \zeta) & =\frac{1}{2} \mathbb{E}\left[\sum_{v \in S^{\circ}} a\left(0, v, \bar{S}^{\mathrm{o}}\right) \nabla f\left(0, v, \bar{S}^{\mathrm{o}}\right) \zeta\left(0, v, \bar{S}^{\mathrm{o}}\right)\right] \\
& =\frac{1}{2} \mathbb{E}\left[\sum_{v \in S^{\circ}} a\left(0, v, \bar{S}^{\mathrm{o}}\right) f\left(v, \bar{S}^{\mathrm{o}}\right) \zeta\left(0, v, \bar{S}^{\mathrm{o}}\right)\right]-\frac{1}{2} \mathbb{E}\left[\sum_{v \in S^{\circ}} a\left(0, v, \bar{S}^{\mathrm{o}}\right) f\left(0, \bar{S}^{\mathrm{o}}\right) \zeta\left(0, v, \bar{S}^{\mathrm{o}}\right)\right] \\
& =\frac{1}{2} \mathbb{E}\left[\sum_{v \in S^{\circ}} a\left(0, v, \bar{S}^{\mathrm{o}}\right) f\left(v, \bar{S}^{\mathrm{o}}\right) \zeta\left(0, v, \bar{S}^{\mathrm{o}}\right)\right]-\frac{1}{2} \mathbb{E}\left[f\left(0, \bar{S}^{\mathrm{o}}\right) \operatorname{div} \zeta\left(0, \bar{S}^{\mathrm{o}}\right)\right] .
\end{aligned}
$$

Basta mostrar então que

$$
\mathbb{E}\left[\sum_{v \in S^{\circ}} a\left(0, v, \bar{S}^{\mathrm{o}}\right) f\left(v, \bar{S}^{\mathrm{o}}\right) \zeta\left(0, v, \bar{S}^{\mathrm{o}}\right)\right]=-\mathbb{E}\left[f\left(0, \bar{S}^{\mathrm{o}}\right) \operatorname{div} \zeta\left(0, \bar{S}^{\mathrm{o}}\right)\right]
$$

Para isso, observe primeiro que se $T\left(v, w, \bar{S}^{\mathrm{o}}\right)=a\left(v, w, \bar{S}^{\mathrm{o}}\right) f\left(w, \bar{S}^{\mathrm{o}}\right) \nabla \zeta\left(v, w, \bar{S}^{\mathrm{o}}\right)$, segue da covariância de $a$ e $\zeta$, e da condição (4.19) que $T$ é covariante. Assim, pelo princípio de transporte de massa (Lema 2.16) segue que

$$
\begin{aligned}
\mathbb{E}\left[\sum_{v \in S^{\circ}} a\left(0, v, \bar{S}^{\circ}\right) f\left(v, \bar{S}^{\circ}\right) \zeta\left(0, v, \bar{S}^{\circ}\right)\right] & =\mathbb{E}\left[\sum_{v \in S^{\circ}} T\left(0, v, \bar{S}^{\circ}\right)\right] \\
& =\mathbb{E}\left[\sum_{v \in S^{\circ}} T\left(v, 0, \bar{S}^{\circ}\right)\right] \\
& =\mathbb{E}\left[\sum_{v \in S^{\circ}} a\left(v, 0, \bar{S}^{\circ}\right) f\left(0, \bar{S}^{\circ}\right) \zeta\left(v, 0, \bar{S}^{\circ}\right)\right] \\
& =-\mathbb{E}\left[\sum_{v \in S^{\circ}} a\left(0, v, \bar{S}^{\circ}\right) f\left(0, \bar{S}^{\circ}\right) \zeta\left(0, v, \bar{S}^{\circ}\right)\right] \\
& =-\mathbb{E}\left[f\left(0, \bar{S}^{\circ}\right) \operatorname{div} \zeta\left(0, \bar{S}^{\circ}\right)\right],
\end{aligned}
$$

onde a penúltima igualdade segue do fato de $\zeta$ ser um fluxo e de $a\left(0, v, \bar{S}^{\circ}\right)=a\left(v, 0, \bar{S}^{\circ}\right)$. 


\subsection{O processo de Harness como uma sequência em $\mathcal{H}$}

Nosso próximo passo é localizar o campo gradiente do processo Harness como uma sequência de campos em $\mathcal{H}$. Mas antes façamos uma análise menos formal do comportamento de $\mathcal{C}\left(\left|\nabla \eta_{t}^{c}\right|^{2}\right)$.

Para isso, fixe momentaneamente uma caixa limitada $A \subset \mathbb{R}^{d}$ e, dado um intervalo de tempo $[t, t+s]$, ordene todas as marcas temporais que ocorrem nos pontos de $S \cap A$ em $[t, t+s]$, ou seja, faça $\{\mathcal{T}(v) \cap[t, t+s] ; v \in S \cap A\}=\left\{t_{1}, \ldots, t_{k}\right\}$, onde $k=|\{\mathcal{T}(v) \cap[t, t+s] ; v \in S \cap A\}|$ e $t<t_{1}<\cdots<t_{k}<t+s$

Suponha que a marca $t_{1}$ acontece no ponto $v$. É um exercício simples verificar que $M_{v}^{S} \eta_{t}^{c}(v)$ minimiza a função $f(x)=\sum_{w \in V(v)}\left|\eta_{t}^{c}(w)-x\right|^{2}$, de modo que

$$
\sum_{w \in V(v)}\left|\nabla \eta_{t_{1}}^{c}(v, w)\right|^{2} \leq \sum_{w \in V(v)}\left|\nabla \eta_{t}^{c}(v, w)\right|^{2}
$$

e portanto

$$
\sum_{v \in S \cap A} \sum_{w \in V(v)}\left|\nabla \eta_{t_{1}}^{c}(v, w)\right|^{2} \leq \sum_{v \in S \cap A} \sum_{w \in V(v)}\left|\nabla \eta_{t}^{c}(v, w)\right|^{2}
$$

Repetindo o mesmo argumento para $t_{2}, \ldots, t_{k}$ concluímos que

$$
\sum_{v \in S \cap A} \sum_{w \in V(v)}\left|\nabla \eta_{t+s}^{c}(v, w)\right|^{2} \leq \sum_{v \in S \cap A} \sum_{w \in V(v)}\left|\nabla \eta_{t}(v, w)\right|^{2}
$$

e por (4.18) teriamos que

$$
\begin{aligned}
\mathcal{C}\left(\left|\nabla \eta_{t+s}^{c}\right|^{2}\right) & =\lim _{A \rightarrow \mathbb{R}^{d}} \frac{1}{2 \ell(A)} \sum_{v \in A \cap S} \sum_{w \in V(v)}\left|\nabla \eta_{t+s}^{c}(v, w)\right|^{2} \\
& \leq \lim _{A \rightarrow \mathbb{R}^{d}} \frac{1}{2 \ell(A)} \sum_{v \in A \cap S} \sum_{w \in V(v)}\left|\nabla \eta_{t}^{c}(v, w)\right|^{2} \\
& =\mathcal{C}\left(\left|\nabla \eta_{t}^{c}\right|^{2}\right) .
\end{aligned}
$$

Existe porém uma falha neste argumento. Ordenamos as marcas associadas aos pontos dentro da caixa, mas nos esquecemos daquelas associadas a pontos na fronteira, i.e., pontos que não pertençam à caixa $A$, mas que possuem ao menos um vizinho em $A$.

Apesar deste problema, a argumentação acima nos dá uma boa idéia do que esperar do campo $\nabla \eta_{t}^{c}$. Ela nos mostra que, se $\nabla \eta_{0}^{c} \in \mathcal{H}$, então $\nabla \eta_{t}^{c} \in \mathcal{H}$, e sequência das normas deve ser decrescente. Isto é novamente coerente com a interpretação de $\mathcal{C}\left(|\zeta|^{2}\right)$ como energia. De fato, se $\nabla \eta_{t}^{c}$ convergir em $\mathcal{H}$, o fato de $\mathcal{C}\left(\left|\nabla \eta_{t}^{c}\right|^{2}\right)$ decrescer é um indício de que seu limite, possivelmente um gradiente de função 
harmônica, pode ser um minimizante local de tal quantidade. Nada disso pode ser afirmado ainda, mas o lema a seguir nos deixa um pouco mais próximos, confirmando a suspeita de que $\mathcal{C}\left(\left|\nabla \eta_{t}^{c}\right|^{2}\right)$ é de fato decrescente, e dando também sua taxa de decaimento.

Como de costume, no lema abaixo consideramos $\pi_{c}(v)=(c \cdot v)$ para algum $c \in \mathbb{R}^{d}$.

Lema 4.3. Para todo $t>0$ e todo $c \in \mathbb{R}^{d}$

$$
\frac{d}{d t} \mathcal{C}\left(\left|\nabla \eta_{t}^{c}\right|^{2}\right)=-2 \mathbb{E}\left[|V(0)|\left|\Delta^{\star} \eta_{t}^{c}\left(0, \bar{S}^{\mathrm{o}}\right)\right|^{2}\right]
$$

onde $\Delta^{\star} \eta_{t}^{c}\left(0, \bar{S}^{\mathrm{o}}\right)=\frac{\Delta \eta_{t}^{c}\left(0, \bar{S}^{\circ}\right)}{|V(0)|}$

Demonstração. Seja

$$
V_{2}:=\bigcup_{v \in V(0)} V(v)
$$

o conjunto de pontos a distância menor ou igual a 2 da origem (na métrica natural de $\mathcal{G}$ ) e

$$
\mathcal{T}_{2}:=\bigcup_{v \in V_{2}} \mathcal{T}(v)
$$

o conjunto de marcas temporais nos pontos de $V_{2}$.

Defina agora os eventos

$$
\begin{gathered}
F_{1}:=F_{1}(t, h)=\left\{\left|\mathcal{T}_{2} \cap[t, t+h]\right|=1\right\} ; \\
F_{1, v}:=F_{1, v}(t, t+h)=F_{1} \cap\{|\mathcal{T}(v) \cap[t, t+h]|=1\} \cap\left\{v \in V_{2}\right\} ; \\
F_{2}:=F_{2}(t, h)=\left\{\left|\mathcal{T}_{2} \cap[t, t+h]\right| \geq 2\right\} .
\end{gathered}
$$

Note que $\mathcal{T}_{2}$ é um processo pontual estacionário em $\mathbb{R}$, dado pela união de uma quantidade aleatória $\left|V_{2}\right|$ de processos de Poisson independentes, ou seja, fixado os pontos de $S^{\mathrm{o}}$, $\mathcal{T}_{2}$ é um processo de Poisson de intensidade $\left|V_{2}\right|$, e portanto, abusando da notação e denotando por $S^{\circ}$ a $\sigma$-álgebra gerada por $S^{\mathrm{o}}$, vale que

$$
\begin{aligned}
& \mathbb{P}\left(F_{1} \mid S^{\mathrm{o}}\right)=\mathbb{E}\left[\mathbf{1}_{F_{1}} \mid S^{\mathrm{o}}\right]=\left|V_{2}\right| h e^{-\left|V_{2}\right| h}, \\
& \mathbb{P}\left(F_{1, v} \mid S^{\mathrm{o}}\right)=h e^{-\left|V_{2}\right| h} \mathbf{1}_{V_{2}}(v),
\end{aligned}
$$


$\mathrm{e}$

$$
\begin{aligned}
\mathbb{P}\left(F_{2} \mid S^{\mathrm{o}}\right) & =\sum_{k \geq 2} \frac{\left(h\left|V_{2}\right|\right)^{k}}{k !} e^{-h\left|V_{2}\right|} \\
& \leq h^{2}\left|V_{2}\right|^{2} \sum_{k \geq 2} \frac{\left(h\left|V_{2}\right|\right)^{k-2}}{(k-2) !} e^{-h\left|V_{2}\right|} \\
& =h^{2}\left|V_{2}\right|^{2}
\end{aligned}
$$

Segue então que

$$
\begin{aligned}
\frac{1}{h} \mathcal{C}\left(\left|\nabla \eta_{t+h}^{c}\right|^{2}-\left|\nabla \eta_{t}^{c}\right|^{2}\right)= & \frac{1}{2 h} \mathbb{E}\left[\sum_{v \in S^{\circ}} a(0, v)\left(\left|\nabla \eta_{t+h}^{c}(0, v)\right|^{2}-\left|\nabla \eta_{t}^{c}(0, v)\right|^{2}\right)\right] \\
= & \frac{1}{2 h} \mathbb{E}\left[\sum_{v \in S^{\circ}} a(0, v)\left(\left|\nabla \eta_{t+h}^{c}(0, v)\right|^{2}-\left|\nabla \eta_{t}^{c}(0, v)\right|^{2}\right) \mathbf{1}_{F_{1}}\right]+ \\
& +\frac{1}{2 h} \mathbb{E}\left[\sum_{v \in S^{\circ}} a(0, v)\left(\left|\nabla \eta_{t+h}^{c}(0, v)\right|^{2}-\left|\nabla \eta_{t}^{c}(0, v)\right|^{2}\right) \mathbf{1}_{F_{2}}\right]
\end{aligned}
$$

Passaremos agora a uma análise caso a caso das possíveis atualizações. Mas antes observe que, fazendo $M_{v}=M_{v}^{S^{\circ}}$,

$$
\Delta^{\star} \eta(v)=\frac{1}{|V(v)|} \sum_{w \in V(v)}(\eta(w)-\eta(v))=M_{v} \eta(v)-\eta(v)
$$

Além disso,

$$
\left|\nabla \eta_{t+h}^{c}(0, v)\right|^{2}-\left|\nabla \eta_{t}^{c}(0, v)\right|^{2}=\left[\nabla \eta_{t+h}^{c}(0, v)-\nabla \eta_{t}^{c}(0, v)\right]^{2}+2 \nabla \eta_{t}^{c}(0, v)\left[\nabla \eta_{t+h}^{c}(0, v)-\nabla \eta_{t}^{c}(0, v)\right]
$$

Vamos a análise. Quando ocorre o evento $F_{1}$, se a marca não acontece na origem, nem em um de seus vizinhos então para todo $v \in V(0)$

$$
\nabla \eta_{t+h}^{c}(0, v)=\nabla \eta_{t}^{c}(0, v)
$$

e diferença é zero. 
Se a marca acontece na origem então, para cada $v \in V(0)$,

$$
\begin{aligned}
\left|\nabla \eta_{t+h}^{c}(0, v)\right|^{2}-\left|\nabla \eta_{t}^{c}(0, v)\right|^{2} & =\left[-M_{0} \eta_{t}^{c}(0)+\eta_{t}^{c}(0)\right]^{2}+2 \nabla \eta_{t}^{c}(0, v)\left[-M_{0} \eta_{t}^{c}(0)+\eta_{t}^{c}(0)\right] \\
& =-2 \nabla \eta_{t}^{c}(0, v) \Delta^{\star} \eta_{t}^{c}(0)+\left|\Delta^{\star} \eta_{t}^{c}(0)\right|^{2}
\end{aligned}
$$

Quando a marca acontece em $v \in V(0)$, o gradiente nos elos não incidentes em $v$ fica inalterado, isto é,

$$
\nabla \eta_{t+h}^{c}(0, w)=\nabla \eta_{t}^{c}(0, w)
$$

para $w \neq v$. Já no elo $(0, v)$ temos

$$
\begin{aligned}
\left|\nabla \eta_{t+h}^{c}(0, v)\right|^{2}-\left|\nabla \eta_{t}^{c}(0, v)\right|^{2} & =\left[M_{v} \eta_{t}^{c}(v)-\eta_{t}^{c}(v)\right]^{2}+2 \nabla \eta_{t}^{c}(0, v)\left[M_{v} \eta_{t}^{c}(v)+\eta_{t}^{c}(v)\right] \\
& =2 \nabla \eta_{t}^{c}(0, v) \Delta^{\star} \eta_{t}^{c}(v)+\left|\Delta^{\star} \eta_{t}^{c}(v)\right|^{2}
\end{aligned}
$$

Observe agora que, dado $S^{\circ}$, o processo $\mathcal{T}_{2} \cap[t, t+h]$ é independente de $\eta_{t}^{c}$, e assim, por (4.25) e (4.26), segue que

$$
\begin{aligned}
\frac{1}{h} \mathbb{E}\left[\sum_{v \in V(0)}\left(\left|\nabla \eta_{t+h}^{c}(0, v)\right|^{2}-\left|\nabla \eta_{t}^{c}(0, v)\right|^{2}\right) \mathbf{1}_{F_{1}}\right] \\
=\frac{1}{h} \mathbb{E}\left[\sum_{w \in V_{2}} \sum_{v \in V(0)}\left(\left|\nabla \eta_{t+h}^{c}(0, v)\right|^{2}-\left|\nabla \eta_{t}^{c}(0, v)\right|^{2}\right) \mathbf{1}_{F_{1, w}}\right] \\
+\frac{1}{h} \mathbb{E}\left[\sum_{v \in V(0)}\left(\left|\nabla \eta_{t+h}^{c}(0, v)\right|^{2}-\left|\nabla \eta_{t}^{c}(0, v)\right|^{2}\right) \mathbf{1}_{F_{1,0}}\right] \\
=\frac{1}{h} \mathbb{E}\left[\sum_{v \in V(0)}\left(2 \nabla \eta_{t}^{c}(0, v) \Delta^{\star} \eta_{t}^{c}(v)+\left|\Delta^{\star} \eta_{t}^{c}(v)\right|^{2}\right) \mathbf{1}_{F_{1, v}}\right] \\
+\frac{1}{h} \mathbb{E}\left[\sum_{v \in V(0)}\left(-2 \nabla \eta_{t}^{c}(0, v) \Delta^{\star} \eta_{t}^{c}(0)+\left|\Delta^{\star} \eta_{t}^{c}(0)\right|^{2}\right) \mathbf{1}_{F_{1,0}}\right]
\end{aligned}
$$


Consequentemente, por (4.21) e (4.22), vale que

$$
\begin{aligned}
\frac{1}{h} \mathbb{E}\left[\sum_{v \in V(0)}\left(\left|\nabla \eta_{t+h}^{c}(0, v)\right|^{2}-\left|\nabla \eta_{t}^{c}(0, v)\right|^{2}\right) \mathbf{1}_{F_{1}}\right] \\
=\frac{1}{h} \mathbb{E}\left[\mathbb{E}\left[\sum_{s \in V(0)}\left(2 \nabla \eta_{t}^{c}(0, v) \Delta^{\star} \eta_{t}^{c}(v)+\left|\Delta^{\star} \eta_{t}^{c}(v)\right|^{2}\right) \mid S^{\mathrm{o}}\right] h \mathrm{e}^{\left.-\left|V_{2}\right| h\right]}\right. \\
\left.+\frac{1}{h} \mathbb{E}\left[\sum_{v \in V(0)}\left(-2 \nabla \eta_{t}^{c}(0, v) \Delta^{\star} \eta_{t}^{c}(0)+\left|\Delta^{\star} \eta_{t}^{c}(0)\right|^{2}\right) \mid S^{\mathrm{o}}\right] h \mathrm{e}^{-\left|V_{2}\right| h}\right] \\
=\mathbb{E}\left[\mathrm{e}^{\left.-\left|V_{2}\right| h \sum_{v \in V(0)}\left(2 \nabla \eta_{t}^{c}(0, v) \nabla \Delta^{\star} \eta_{t}^{c}(0, v)+\left|\Delta^{\star} \eta_{t}^{c}(v)\right|^{2}+\left|\Delta^{\star} \eta_{t}^{c}(0)\right|^{2}\right)\right]}\right.
\end{aligned}
$$

Pelo teorema da convergência monótona e pelo princípio de transporte de massa (lema 2.16), segue então que

$$
\begin{gathered}
\lim _{h \rightarrow 0} \frac{1}{2 h} \mathbb{E}\left[\sum_{v \in V(0)}\left(\left|\nabla \eta_{t+h}^{c}(0, v)\right|^{2}-\left|\nabla \eta_{t}^{c}(0, v)\right|^{2}\right) \mathbf{1}_{F_{1}}\right]= \\
2 \mathcal{C}\left(\nabla \eta_{t}^{c} \nabla \Delta^{\star} \eta_{t}^{c}\right)+\frac{1}{2} \mathbb{E}\left[\sum_{v \in V(0)}\left(\left|\Delta^{\star} \eta_{t}^{c}(v)\right|^{2}+\left|\Delta^{\star} \eta_{t}^{c}(0)\right|^{2}\right)\right] \\
=2 \mathcal{C}\left(\nabla \eta_{t}^{c} \nabla \Delta^{\star} \eta_{t}^{c}\right)+\mathbb{E}\left(|V(0)|\left|\Delta^{\star} \eta_{t}^{c}\right|^{2}\right)
\end{gathered}
$$

Antes de analisarmos o segundo termo, observe que

$$
\mathcal{C}\left(\left|\nabla \eta_{t}^{c}\right|^{r}\right) \leq \mathcal{C}\left(\left|\nabla \pi_{c}\right|^{r}\right)+\mathcal{C}\left(\left|\nabla \phi_{t}\right|^{r}\right)
$$

$\mathrm{e}$

$$
\mathcal{C}\left(\left|\nabla \phi_{t}\right|^{r}\right) \leq \mathbb{E}\left[\sum_{v \in V(0)}\left|\phi_{t}(v)-\phi_{t}(0)\right|^{r}\right] \leq \mathbb{E}\left[|V(0)|\left|\phi_{t}(0)\right|^{r}\right] \leq C M_{r}(t)
$$

Passemos agora a analise do segundo termo de (4.24). Para isso, considere o campo $\zeta_{h}$ dado por

$$
\zeta_{h}\left(v, w, \bar{S}^{\mathrm{o}}\right)=\mathbf{1}_{F_{2}}\left(\tau_{v} \bar{S}^{\mathrm{o}}\right),
$$


e observe que

$$
\begin{aligned}
\frac{1}{h} \mathbb{E}\left[\sum_{v \in V(0)}\left|\nabla \eta_{s}^{c}(0, v)\right|^{2} \mathbf{1}_{F_{2}}\right] & =\frac{1}{h} \mathcal{C}\left(\left|\nabla \eta_{s}^{c}\right|^{2} \zeta_{h}\right) \\
& \leq \frac{1}{h}\left[\mathcal{C}\left(\left|\nabla \eta_{s}^{c}\right|^{2 p}\right)\right]^{\frac{1}{p}}\left[\mathcal{C}\left(\zeta_{h}\right)\right]^{\frac{1}{q}} \\
& \leq \frac{1}{h}\left[\mathcal{C}\left(\left|\nabla \eta_{s}^{c}\right|^{2 p}\right)\right]^{\frac{1}{p}}\left[\mathbb{E}\left[|V(0)| \mathbf{1}_{F_{2}}\right]\right]^{\frac{1}{q}} \\
& \leq\left[C+C M_{2 p}(s)\right]^{\frac{1}{p}} \mathbb{E}\left[\left|V_{2}\right|^{3}\right]^{\frac{1}{q}} h^{\frac{2}{q}-1}
\end{aligned}
$$

De onde segue que

$$
\begin{aligned}
\frac{1}{h}\left|\mathbb{E}\left[\sum_{v \in V(0)}\left(\left|\nabla \eta_{t+h}^{c}(0, v)\right|^{2}-\left|\nabla \eta_{t}^{c}(0, v)\right|^{2}\right) \mathbf{1}_{F_{2}}\right]\right| \\
\leq\left(\left[C+C M_{2 p}(t+h)\right]^{\frac{1}{p}}+\left[C+C M_{2 p}(t)\right]^{\frac{1}{p}}\right) \mathbb{E}\left[\left|V_{2}\right|^{3}\right]^{\frac{1}{q}} h^{\frac{2}{q}-1}
\end{aligned}
$$

e portanto, tomando $q<2$ (e consequentemente $p>2$ )

$$
\lim _{h \rightarrow 0} \frac{1}{2 h} \mathbb{E}\left[\sum_{v \in V(0)}\left(\left|\nabla \eta_{t+h}^{c}(0, v)\right|^{2}-\left|\nabla \eta_{t}^{c}(0, v)\right|^{2}\right) \mathbf{1}_{F_{2}}\right]=0 .
$$

De (4.24), (4.27), (4.28) e da fórmula de integração por partes, segue que

$$
\begin{aligned}
\frac{d}{d t} \mathcal{C}\left(\left|\nabla \eta_{t}^{c}\right|^{2}\right) & =2 \mathcal{C}\left(\nabla \eta_{t}^{c} \nabla \Delta^{\star} \eta_{t}^{c}\right)+\mathbb{E}\left[\left|V(0) \| \Delta^{\star} \eta_{t}^{c}\right|^{2}\right] \\
& =-\mathbb{E}\left[\left|V(0) \| \Delta^{\star} \eta_{t}^{c}\right|^{2}\right]
\end{aligned}
$$

Do Lema 4.3 segue diretamente que

Corolário 4.4. $\mathcal{C}\left(\left|\nabla \eta_{t}^{c}\right|^{2}\right)$ é decrescente em $t$, sendo constante a partir do instante $t \geq 0$ se, e somente se, $\eta_{t}^{c}$ for harmônica em $\mathcal{G}\left(S^{\circ}\right)$.

Outra consequência (não tão direta) do Lema 4.3 é dada no seguinte resultado. 
Lema 4.5. Se $S^{\circ}$ satisfaz as hipóteses do Lema 4.3, então

$$
|V(0)|^{-1} \Delta \eta_{t}^{c}\left(0, \bar{S}^{\mathrm{o}}\right) \rightarrow 0, \quad \text { quando } t \rightarrow \infty
$$

quase-certamente e em $L^{2}$.

Demonstração. Para simplificar a notação, faça

$$
Z_{t}:=\frac{\left|\Delta \eta_{t}^{c}\left(0, \bar{S}^{\mathrm{o}}\right)\right|^{2}}{|V(0)|}=|V(0)|\left|\Delta^{\star} \eta_{t}^{c}\left(0, \bar{S}^{\mathrm{o}}\right)\right|^{2}
$$

Seja $\mathcal{T}_{1}$ o processo pontual estacionário em $\mathbb{R}^{+}$dado por

$$
\mathcal{T}_{1}=\mathcal{T}(0) \cup\left\{\bigcup_{v \in V(0)} \mathcal{T}(v)\right\}
$$

onde $\mathcal{T}(v)$ é o processo de marcas temporais associadas a $v \in S^{\circ}$ definido na construção do processo de harness.

Como para cada $v \in S^{\circ}$ o processo $\mathcal{T}(v)$ é um processo de Poisson de taxa 1 , então, dado $S^{\circ}, \mathcal{T}_{1}$ é um processo de Poisson de intensidade $|V(0)|+1$. Enumere os pontos de $\mathcal{T}_{1}$ por $0<t_{1}<t_{2}<\cdots$. Para simplificar a notação, faça $t_{0}=0$, mas lembre que isto não significa que $\mathcal{T}_{1}$ tem um ponto na origem. Observe que, para cada $n \geq 0, Z_{t_{n}}$ depende apenas de $t_{k}$ para $k \leq n$ e, portanto, dado $S^{\circ}$, $Z_{t_{n}}$ é independente de $\left(t_{n+1}-t_{n}\right)$.

Segue que

$$
\begin{aligned}
\int_{0}^{\infty} \mathbb{E}\left[Z_{t}\right] d t & =\mathbb{E}\left[\int_{0}^{\infty} Z_{t} d t\right] \\
& =\sum_{k=0}^{\infty} \mathbb{E}\left[Z_{t_{k}}\left(t_{k+1}-t_{k}\right)\right] \\
& =\sum_{k=0}^{\infty} \mathbb{E}\left[\mathbb{E}\left[Z_{t_{k}} \mid S^{\mathrm{o}}\right] \frac{1}{|V(0)|+1}\right] \\
& =\sum_{k=0}^{\infty} \mathbb{E}\left[\frac{Z_{t_{k}}}{|V(0)|+1}\right]
\end{aligned}
$$

de onde concluímos que, pelo Lema 4.3

$$
\mathbb{E}\left[\sum_{k=0}^{\infty} \frac{Z_{t_{k}}}{|V(0)|+1}\right]<\infty
$$


Em particular,

$$
\sum_{k=0}^{\infty} \Delta \eta_{t_{k}}\left(0, \bar{S}^{\mathrm{o}}\right)<\infty \quad \text { q.c. }
$$

$\mathrm{e}$

$$
\lim _{t \rightarrow \infty} \Delta \eta_{t}\left(0, \bar{S}^{\mathrm{o}}\right)=0 \quad \text { q.c.. }
$$

Para a convergência em $L_{2}$ note primeiro que, para todo $t \geq 0,\left|\Delta^{\star} \eta_{t}^{c}(0)\right|^{2} \leq \sum_{n \geq 0}\left|\Delta^{\star} \eta_{t_{n}}^{c}(0)\right|^{2}$. Assim, pelo teorema da convergência dominada, e por (4.30)

$$
\lim _{t \rightarrow \infty} \mathbb{E}\left[\left|\Delta^{\star} \eta_{t}^{c}\left(0, \bar{S}^{\mathrm{o}}\right)\right|^{2}\right]=0
$$

\subsubsection{Inclinação}

Vamos começar agora a discutir a linearidade do nosso possível limite. Nesta seção vamos nos concentrar em encontrar uma forma de caracterizar $I_{u}(\cdot)$ para funções com gradiente em $\mathcal{H}$. Neste sentido, dada uma direção $u \in \mathbb{R}^{d},\|u\|=1$, e $v, w \in S^{\circ}$ tais que $w \in V(v)$, seja $b\left(v, w, S^{\circ}\right)$ a face $(d-1)$-dimensional comum às células de Voronoi de $v$ e $w$, e denote por $b_{u}\left(v, w, S^{\circ}\right)$ a medida de lebesgue $d$-1-dimensional da projeção $p_{u}\left(v, w, S^{\circ}\right)$ de $b\left(v, w ; S^{\circ}\right)$ sobre o hiperplano perpendicular a $u$. Defina agora

$$
\zeta_{u}\left(v, w, S^{\mathrm{o}}\right):=\frac{1}{2} \operatorname{sg}([w-v] \cdot u) b_{u}\left(v, w, S^{\mathrm{o}}\right)
$$

onde $\operatorname{sg}(x)=\frac{x}{|x|}, x \in \mathbb{R}$.

A Proposição a seguir dá a caracterização que buscamos, fornecendo uma maneira fácil de calcular a inclinação de funções com gradiente covariante.

Proposição 4.6. Seja $\eta: \Xi_{1} \rightarrow \mathbb{R}$ com $\nabla \eta \in \mathcal{H}$ covariante. Então, dada uma direção $u \in \mathbb{R}^{d}$

$$
I_{u}(\eta)=\mathcal{C}\left(\nabla \eta \cdot \zeta_{u}\right) \quad \text { q.c.. }
$$

Para demonstrar a Proposição 4.6 será necessário que mostremos antes um resultado técnico, mas importante.

Para $y \in \mathbb{R}^{d-1}$, seja $l_{y}=\left\{(0, y)+\alpha e_{1} ; \alpha \in \mathbb{R}\right\}$ a reta passando por $(0, y) \in \mathbb{R}^{d}$ na direção $e_{1}=(1,0, \ldots, 0) \in \mathbb{R}^{d}$. Fixado $\xi \in \mathcal{N}$, defina $L(y, \xi):=\left\{v \in \xi: \mathrm{C}(v) \cap l_{y} \neq \emptyset\right\}$. Agora, para $x=\left(x_{1}, \ldots, x_{d}\right) \in \mathbb{R}^{d}$, seja $x^{*}=\left(x_{2}, \ldots, x_{d}\right) \in \mathbb{R}^{d-1}$. 


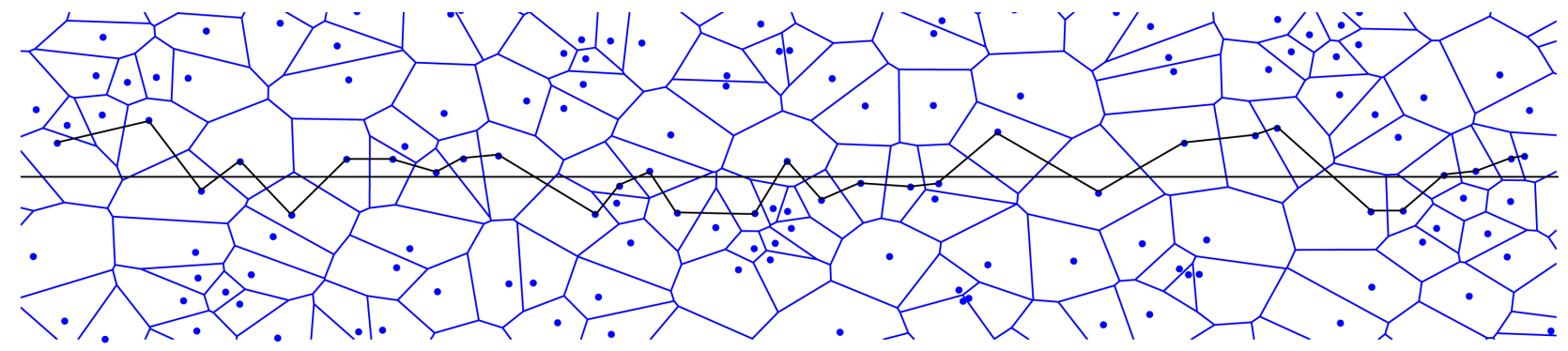

Figura 4.1: Pontos de $L(0, \xi)$ ao longo da direção coordenada $e_{1}$.

Defina agora $\omega: \mathbb{R}^{d-1} \times \Xi_{2} \rightarrow \mathbb{R}$ e $\theta: \mathbb{R}^{d-1} \times \Xi_{1} \rightarrow \mathbb{R}$ por

$$
\omega(y ; v, w, \xi)= \begin{cases}1, & \text { se } v, w \in L(y), b(v, w, \xi) \cap l_{y} \neq \emptyset ; \\ 0, & \text { caso contrário }\end{cases}
$$

e

$$
\theta(y ; v, \xi)=\sum_{w \in \xi} w a^{+}(v, w, \xi) \omega(y ; v, w, \xi)
$$

onde $a^{+}(v, w, \xi)=a(v, w, \xi) \mathbf{1}\{(w \cdot u)>(v \cdot u)\}$. Em palavras, $\theta(y ; v, \xi)$ é o vizinho de $v$, que está "a frente" de $v$ na direção $u$, e cuja face da célula de Voronoi compartilhada com $\mathrm{C}(v)$ é cruzada pela reta $l_{y}$.

Observe que

$$
w(y ; v, w, \xi)=w\left(y-x^{*} ; v-x, w-x, \tau_{x} \xi\right),
$$

e

$$
\theta(y ; v, \xi)-x=\theta\left(y-x^{*} ; v-x, \tau_{x} \xi\right)
$$

para todo $x \in \mathbb{R}^{d}$.

Lema 4.7. Se $\zeta \in \mathcal{H}$ é um fluxo então

$$
\mathbb{E}\left[\sum_{v \in S} \zeta(v, \theta(0 ; v, S), S) \mathbf{1}_{L(0, S)}(v) \mathbf{1}_{A}\left(v_{1}\right)\right]=\ell(A) \mathcal{C}\left(\zeta \cdot \zeta_{e_{1}}\right)
$$

para todo $A \in \mathcal{B}(\mathbb{R})$ com medida de lebesgue $\ell(A)<\infty$.

Demonstração. Defina

$$
g(v, S):=|\zeta(v, \theta(0 ; v, S), S)| \mathbf{1}_{L(0, S)}(v) \mathbf{1}_{A}\left(v_{1}\right) .
$$


Segue então de (4.32), (4.33), da Fórmula de Campbell Generalizada (Proposição 2.14), e por Fubini que

$$
\begin{aligned}
\mathbb{E}\left[\sum_{v \in S} g(v, S)\right] & =\int_{\mathbb{R}^{d}} \mathbb{E}^{o}\left[g\left(v, \tau_{-v} S^{\mathrm{o}}\right)\right] d v \\
& =\int_{\mathbb{R}^{d}} \mathbb{E}^{o}\left[\left|\zeta\left(v, \theta\left(0 ; v, \tau_{-v} S^{\mathrm{o}}\right), \tau_{-v} S^{\mathrm{o}}\right)\right| \mathbf{1}_{L\left(0, \tau_{-v} S^{\circ}\right)}(v) \mathbf{1}_{A}\left(v_{1}\right)\right] d v \\
& =\int_{\mathbb{R}^{d}} \mathbb{E}^{o}\left[\left|\zeta\left(0, \theta\left(-v^{*} ; 0, S^{\mathrm{o}}\right), S^{\mathrm{o}}\right)\right| \mathbf{1}_{L\left(-v^{*}, S^{\circ}\right)}(0) \mathbf{1}_{A}\left(v_{1}\right)\right] d v \\
& =\ell(A) \int_{\mathbb{R}^{d-1}} \mathbb{E}^{o}\left[\left|\zeta\left(0, \theta\left(-v^{*} ; 0, S^{\mathrm{o}}\right), S^{\mathrm{o}}\right)\right| \mathbf{1}_{L\left(-v^{*}, S^{\circ}\right)}(0)\right] d v^{*} \\
& =\ell(A) \int_{\mathbb{R}^{d-1}} \mathbb{E}^{o}\left[\sum_{w \in S^{\circ}}\left|\zeta\left(0, w, S^{\mathrm{o}}\right)\right| \mathbf{1}\left\{\theta\left(-v^{*} ; 0, S^{\mathrm{o}}\right)=w\right\} \mathbf{1}_{L\left(-v^{*}, S^{\circ}\right)}(0)\right] d v^{*} \\
& =\ell(A) \mathbb{E}^{o}\left[\sum_{w \in S^{\circ}}\left|\zeta\left(0, w, S^{\mathrm{o}}\right)\right| \int_{\mathbb{R}^{d-1}} \mathbf{1}\left\{\theta\left(-v^{*} ; 0, S^{\mathrm{o}}\right)=w\right\} \mathbf{1}_{L\left(-v^{*}, S^{\circ}\right)}(0) d v^{*}\right] \\
& =\ell(A) \mathbb{E}^{o}\left[\sum_{w \in S^{\circ}}\left|\zeta\left(0, w, S^{\mathrm{o}}\right)\right| \int_{\mathbb{R}^{d-1}} \mathbf{1}\left\{\theta\left(v^{*} ; 0, S^{\mathrm{o}}\right)=w\right\} \mathbf{1}_{L\left(-v^{*}, S^{\circ}\right)}(0) d v^{*}\right]
\end{aligned}
$$

Mas para $w \in S^{\circ}$ tal que $a^{+}\left(0, w, S^{\circ}\right)=1$, vale que

$$
\left\{w=\theta\left(-v^{*} ; 0, S^{\mathrm{o}}\right), 0 \in L\left(-v^{*}, S^{\mathrm{o}}\right)\right\}=\left\{l_{-v^{*}} \cap b\left(0, w, S^{\mathrm{o}}\right) \neq \emptyset\right\}
$$

isto é, $-v^{*} \in p_{e_{1}}\left(0, w, S^{\circ}\right)$. Deste modo

$$
\int_{\mathbb{R}^{d-1}} \mathbf{1}\left\{\theta\left(-v^{*} ; 0, S^{\circ}\right)=w\right\} \mathbf{1}_{L\left(-v^{*}, S^{\circ}\right)}(0) d v^{*}=\int_{\mathbb{R}^{d-1}} \mathbf{1}_{\left\{p_{e_{1}}\left(0, w, S^{\circ}\right)\right\}}\left(-v^{*}\right) d v^{*}=\zeta_{e_{1}}\left(0, w, S^{\mathrm{o}}\right) .
$$

Segue que

$$
\mathbb{E}\left[\sum_{v \in S} g(v, S)\right]=\ell(A) \mathbb{E}^{o}\left[\sum_{w \in S^{\circ}} a^{+}\left(0, w, S^{\mathrm{o}}\right)\left|\zeta\left(0, w, S^{\mathrm{o}}\right)\right| \zeta_{e_{1}}\left(0, w, S^{\mathrm{o}}\right)\right]<\infty .
$$

Agora que sabemos que $\mathbb{E}\left[\sum_{v \in S} g(v, S)\right]<\infty$, podemos repetir os mesmos argumentos para mostrar que

$$
\mathbb{E}\left[\sum_{v \in S} g(v, S)\right]=\ell(A) \mathbb{E}^{o}\left[\sum_{w \in S^{\circ}} a^{+}\left(0, w, S^{\mathrm{o}}\right)\left|\zeta\left(0, w, S^{\mathrm{o}}\right)\right| \zeta_{e_{1}}\left(0, w, S^{\mathrm{o}}\right)\right]
$$


Aplicando agora o princípio de transporte de massa (Lema 2.16) para $a^{+} \cdot \zeta \cdot \zeta_{e_{1}}$, e notando que

$$
a^{+}\left(v, 0, S^{\mathrm{o}}\right) \zeta\left(v, 0, S^{\mathrm{o}}\right) \zeta_{e_{1}}\left(v, 0, S^{\mathrm{o}}\right)=a^{-}\left(0, v, S^{\mathrm{o}}\right) \zeta\left(0, v, S^{\mathrm{o}}\right) \zeta_{e_{1}}\left(0, v, S^{\mathrm{o}}\right)
$$

onde $a^{-}(v, w, \xi)=a(v, w, \xi) \mathbf{1}\{(w \cdot u)<(v \cdot u)\}$, segue que

$$
\begin{aligned}
\mathbb{E}\left[\sum_{v \in S} g(v, S)\right] & =\ell(A) \mathbb{E}^{o}\left[\sum_{w \in S^{\circ}} a^{+}\left(0, w, S^{\mathrm{o}}\right) \zeta\left(0, w, S^{\mathrm{o}}\right) \zeta_{e_{1}}\left(0, w, S^{\mathrm{o}}\right)\right] \\
& =\ell(A) \mathbb{E}^{o}\left[\sum_{w \in S^{\circ}} a^{+}\left(w, 0, S^{\mathrm{o}}\right) \zeta\left(w, 0, S^{\mathrm{o}}\right) \zeta_{e_{1}}\left(w, 0, S^{\mathrm{o}}\right)\right] \\
& =\ell(A) \mathbb{E}^{o}\left[\sum_{w \in S^{\circ}} a^{-}\left(0, w, S^{\mathrm{o}}\right) \zeta\left(0, w, S^{\mathrm{o}}\right) \zeta_{e_{1}}\left(0, w, S^{\mathrm{o}}\right)\right]
\end{aligned}
$$

e portanto

$$
\begin{aligned}
\mathbb{E}\left[\sum_{v \in S} g(v, S)\right] & =\ell(A) \frac{1}{2} \mathbb{E}^{o}\left[\sum_{w \in S^{\circ}} a\left(0, w, S^{\mathrm{o}}\right) \zeta\left(0, w, S^{\mathrm{o}}\right) \zeta_{e_{1}}\left(0, w, S^{\mathrm{o}}\right)\right] \\
& =\ell(A) \mathcal{C}\left(\nabla \eta \cdot \zeta_{e_{1}}\right) .
\end{aligned}
$$

Estamos prontos para demonstrar a Proposição 4.6.

Demostração da Proposição 4.6. Considere inicialmente que $t$ varia de maneira discreta, ou seja, $t \in \mathbb{Z}+$. Para deixar isto claro, escreveremos $K$ ao invés de $t$.

Primeiro observe que, pela covariância de $\nabla \eta$, temos

$\eta\left(\operatorname{Cen}(v+K u), S^{\mathrm{o}}\right)-\eta\left(v, S^{\mathrm{o}}\right)=\eta\left(\operatorname{Cen}(v+K u)-v, \tau_{v} S^{\mathrm{o}}\right)-\eta\left(0, \tau_{v} S^{\mathrm{o}}\right)=\eta\left(\operatorname{Cen}(K u), \tau_{v} S^{\mathrm{o}}\right)-\eta\left(0, \tau_{v} S^{\mathrm{o}}\right)$. Agora, como $S^{o}$ é ponto-estacionário, segue que, se o limite em (1.10) existe, ele não depende de $v \in S^{\mathrm{o}}$.

Seguindo a diante considere, sem perda de generalidade, $u=e_{1}$. Faça $A_{K}:=[0, K] \times \mathbb{R}^{d-1} \mathrm{e}$ defina

$$
\bar{v}_{K}=\operatorname{argmax}\left\{\left(v \cdot e_{1}\right): v \in L\left(0, S^{\mathrm{o}}\right) \cap A_{K}\right\}
$$

e

$$
v_{K}=\theta\left(0 ; \bar{v}_{K}, S^{\mathrm{o}}\right)
$$


ou seja, $v_{K}$ é o primeiro ponto de $L\left(0, S^{\mathrm{o}}\right)$ "a direita" de $A_{K}$.

Faça agora $Z_{K}=\eta\left(\operatorname{Cen}\left(K u, S^{\circ}\right), S^{\circ}\right)-\eta\left(v_{K}\left(S^{\circ}\right), S^{\circ}\right)$ e observe que

$$
\eta\left(\operatorname{Cen}(K u), S^{\mathrm{o}}\right)-\eta\left(0, S^{\mathrm{o}}\right)=\sum_{v \in L\left(0, S^{\circ}\right) \cap A_{K}}\left(\eta\left(\theta\left(0 ; v, S^{\mathrm{o}}\right), S^{\mathrm{o}}\right)-\eta\left(v, S^{\mathrm{o}}\right)\right)+Z_{K}
$$

Primeiro observe que, usando a equação $(4.36), I_{u}(\eta)$ pode ser reescrito como

$$
\begin{aligned}
I_{u}(\eta) & =\lim _{K \rightarrow \infty} \frac{1}{K}\left[\sum_{v \in L\left(0, S^{\circ}\right) \cap A_{K}} \nabla \eta\left(v, \theta\left(0 ; v, S^{\mathrm{o}}\right), S^{\mathrm{o}}\right)+Z_{K}\right] \\
& =\lim _{K \rightarrow \infty} \frac{1}{K}\left[\sum_{v \in S^{\circ}} \nabla \eta\left(v, \theta\left(0 ; v, S^{\mathrm{o}}\right), S^{\mathrm{o}}\right) \mathbf{1}_{L\left(0, S^{\circ}\right)}(v) \mathbf{1}_{[0, K]}\left(v_{1}\right)+Z_{K}\right],
\end{aligned}
$$

e portanto precisamos mostrar que

$$
\tilde{I}:=\lim _{K \rightarrow \infty} \frac{1}{K}\left[\sum_{v \in \xi} \nabla \eta(v, \theta(0 ; v, \xi), \xi) \mathbf{1}_{L(0, \xi)}(v) \mathbf{1}_{[0, K]}\left(v_{1}\right)+Z_{K}\right]=\mathcal{C}\left(\nabla \eta \cdot \gamma_{u}\right),
$$

$\mu_{\mathrm{o}}$-quase certamente, onde $\mu_{\mathrm{o}}$ é a lei de $S^{\mathrm{o}}$.

Defina então $\Gamma=\left\{\xi \in \mathcal{N}: \tilde{I}=\mathcal{C}\left(\nabla \eta \cdot \zeta_{u}\right)\right\}$. Da covariância de $\nabla \eta$, da definição de $L(\cdot, \xi)$ e de (4.33), segue que o evento $\Gamma$ é invariante pela ação de $\tau_{u}=\tau_{e_{1}}$. Portanto, da Proposição 2.12 concluímos que

$$
\mu(\Gamma)=\lim _{k \rightarrow \infty} \mu_{\mathrm{o}}\left(\tau_{k u} \Gamma\right)=\mu_{\mathrm{o}}(\Gamma) .
$$

Assim, mostrar que $\mu_{\mathrm{o}}(\Gamma)=1$ é o mesmo que mostrar que $\mu(\Gamma)=1 \mathrm{e}$, por isso, podemos então deixar de lado o ponto na origem, e trabalhar apenas com o processo estacionário $S$ de lei $\mu$.

Defina $T: \mathcal{N} \rightarrow \mathcal{N}$ por $T(\xi):=\tau_{e_{1}} \xi$. Observe que $T$ possui inversa $T^{-1}:=\tau_{-e_{1}}$. Se $S$ é mixing, segue que para $U_{1}, U_{2} \in \mathcal{N}$

$$
\lim _{k \rightarrow \infty}\left|\mu\left(U_{1} \cap T^{k} U_{2}\right)-\mu\left(U_{1}\right) \mu\left(U_{2}\right)\right|=0,
$$

e portanto $\mu$ é mixing também para $T$.

$$
\text { Seja } \phi(S)=\sum_{s \in S} \nabla \eta(s, \theta(0 ; s, S), S) u(0 ; s, S) \mathbf{1}_{\{[0,1]\}}\left(s_{1}\right) \text {. Note que } L(0, S)=L\left(0, T^{k}\left(S^{\mathrm{o}}\right)\right) \text { e }
$$


portanto, de (4.33) e da covariância de $\nabla \eta$, segue que

$$
\begin{aligned}
\phi \circ T^{k}(S) & =\sum_{v \in T^{k}(S)} \nabla \eta\left(v, \theta\left(0 ; v, T^{k}(S)\right), T^{k}(S)\right) \mathbf{1}_{L\left(0, T^{k}(S)\right)}(v) \mathbf{1}_{[0,1]}\left(v_{1}\right) \\
& =\sum_{v \in S \cap L(0, S)} \nabla \eta\left(v-k e_{1}, \theta\left(0 ; v-k e_{1}, \tau_{k e_{1}} S\right), \tau_{k e_{1}} S\right) \mathbf{1}_{[0,1]}\left(v_{1}-k\right) \\
& =\sum_{v \in S \cap L(0, S)} \nabla \eta\left(v, \theta\left(0 ; v-k e_{1}, \tau_{k e_{1}} S\right)+k e_{1}, S\right) \mathbf{1}_{[0,1]}\left(v_{1}-k\right) \\
& =\sum_{v \in S \cap L(0, S)} \nabla \eta(v, \theta(0 ; v, S), S) \mathbf{1}_{[k, k+1]}\left(v_{1}\right)
\end{aligned}
$$

e com isso

$$
\begin{aligned}
\sum_{s \in S} \nabla \eta(s, \theta(0 ; s, S), S) \omega(0 ; s, S) \mathbf{1}_{[0, K]}\left(s_{1}\right) & =\sum_{k=0}^{K-1} \sum_{s \in S} \nabla \eta(s, \theta(0 ; s, S), S) \omega(0 ; s, S) \mathbf{1}_{[k, k+1]}\left(s_{1}\right) \\
& =\sum_{k=0}^{K-1} \phi \circ T^{k}(S) .
\end{aligned}
$$

Segue do Lema 4.7 e do Teorema Ergódico de Birkoff que

$$
\lim _{K \rightarrow \infty} \frac{1}{K} \sum_{k=0}^{K-1} \phi \circ T^{k}(S)=\mathbb{E}^{o}\left[\phi\left(S^{\circ}\right)\right]=\mathcal{C}\left(\nabla \eta \cdot \gamma_{u}\right) \quad \text { a.s.. }
$$

Só nos resta mostar que

$$
\lim _{K \rightarrow \infty} \frac{Z_{K}}{K}=0
$$

quase certamente. Para isso observe agora que

$$
\begin{aligned}
Z_{k}(S) & =\eta\left(\operatorname{Cen}\left(k e_{1}, S\right)-k e_{1}, \tau_{k e_{1}} S\right)-\eta\left(v_{k}(S)-k e_{1}, \tau_{k e_{1}} S\right) \\
& =\eta\left(\operatorname{Cen}\left(0, \tau k e_{1} S\right), \tau_{k e_{1}} S\right)-\eta\left(v_{0}\left(\tau_{k e_{1}} S\right), \tau_{k e_{1}} S\right) \\
& =Z_{0} \circ T^{k}(S) .
\end{aligned}
$$

Sabemos também que $\mathbb{E}\left(\left|Z_{0}(S)\right|\right)=\mathbb{E}\left[\ell(\mathrm{C}(0))\left|Z_{0}\left(S^{\mathrm{o}}\right)\right|\right]$. Mas $\operatorname{Cen}\left(0, S^{\mathrm{o}}\right)=0$, e portanto $v_{0}\left(S^{\mathrm{o}}\right) \in$ $V\left(\operatorname{Cen}\left(0, S^{\circ}\right)\right)$, de onde segue que

$$
\mathbb{E}\left[\ell(\mathrm{C}(0)) Z_{0}\left(S^{\mathrm{o}}\right)\right] \leq \mathbb{E}\left[\ell(\mathrm{C}(0)) \sum_{v \in V(0)}\left|\nabla \eta\left(0, v, S^{\mathrm{o}}\right)\right|\right]=\mathcal{C}(|\nabla \eta| \cdot \zeta)
$$


onde $\zeta(v, w, \xi)=\ell\left(\mathrm{C}\left(0, \tau_{v} \xi\right)\right)$. Pela desigualdade de Hölder segue então que

$$
\mathbb{E}\left(\left|Z_{0}\left(S^{\mathrm{o}}\right)\right|\right)<\infty
$$

Do Teorema de Birkhoff concluímos portanto que

$$
\frac{1}{K} \sum_{k=0}^{K} Z_{k} \rightarrow \mathbb{E}\left[Z_{0}\left(S^{\mathrm{o}}\right)\right],
$$

quase-certamente, quando $K \rightarrow \infty$, e assim

$$
\lim _{K \rightarrow 0} \frac{Z_{K}}{K}=0
$$

quase certamente, e o resultado segue.

Para o caso de $t$ variando continuamente, ou seja, $t \in \mathbb{R}^{+}$basta notar que

$$
\eta\left(\operatorname{Cen}\left(t u, S^{\mathrm{o}}\right)\right)-\eta(0)=\eta\left(\operatorname{Cen}\left(t u, S^{\mathrm{o}}\right)\right)-\eta\left(\operatorname{Cen}\left([t] u, S^{\mathrm{o}}\right)\right)+\eta\left(\operatorname{Cen}\left([t] u, S^{\mathrm{o}}\right)-\eta(0),\right.
$$

onde $[t]$ é o maior inteiro menor ou igual a $[t]$, e que

$$
\left|\eta\left(\operatorname{Cen}\left(t u, S^{\mathrm{o}}\right)\right)-\eta\left(\operatorname{Cen}\left([t] u, S^{\mathrm{o}}\right)\right)\right| \leq \sum_{v \in L\left(0, S^{\circ}\right)}\left|\nabla \eta\left(v, \theta\left(0 ; v, S^{\mathrm{o}}\right), S^{\mathrm{o}}\right)\right| \mathbf{1}_{[[t],[t]+1]}\left(v_{1}\right)+Z_{[t]+1}
$$

Portanto

$$
\lim _{t \rightarrow \infty} \frac{1}{t}\left|\eta\left(\operatorname{Cen}\left(t u, S^{\mathrm{o}}\right)\right)-\eta\left([t] u, S^{\mathrm{o}}\right)\right|=0
$$

e

$$
\lim _{t \rightarrow \infty} \frac{1}{t}\left[\eta\left(\operatorname{Cen}\left(t u, S^{\mathrm{o}}\right)\right)-\eta(0)\right]=\lim _{t \rightarrow \infty} \frac{1}{t}\left[\eta\left(\operatorname{Cen}\left([t] u, S^{\mathrm{o}}\right)\right)-\eta(0)\right]=\mathcal{C}\left(\nabla \eta \cdot \zeta_{u}\right)
$$

quase certamente.

Tendo em mãos a Proposição anterior podemos mostrar que

Lema 4.8. Dado $c \in \mathbb{R}^{d}$ e $u \in \mathbb{R}^{d}, I_{u}\left(\eta_{t}^{c}-\pi_{c}\right)=0$, quase-certamente, para todo $t \geq 0$. 
Demonstração. Primeiro note que

$$
\operatorname{div} \zeta_{u}=\sum_{v \in V(0)} \zeta_{u}\left(0, v, S^{\mathrm{o}}\right)=\sum_{\substack{v \in V(0) \\(v \cdot u)>0}} p_{u}\left(0, v, S^{\mathrm{o}}\right)-\sum_{\substack{v \in V(0) \\(v \cdot u)<0}} p_{u}\left(0, v, S^{\mathrm{o}}\right)=0, \quad \text { q.c. }
$$

pois

$$
\sum_{\substack{v \in V(0) \\(v \cdot u)>0}} p_{u}\left(0, v, S^{\mathrm{o}}\right)=\sum_{\substack{v \in V(0) \\(v \cdot u)<0}} p_{u}\left(0, v, S^{\mathrm{o}}\right)=V_{u}
$$

onde $V_{u}$ denota a medida de Lebesgue $d-1$ dimensional da projeção da célula de Voronoi da origem no hiperplano ortogonal a $u$.

Segue agora da Proposição 4.6 que, para todo $t \geq 0$

$$
\begin{aligned}
I_{u}\left(\eta_{t}^{c}\right) & =\mathcal{C}\left(\nabla \eta_{t}^{c} \cdot \zeta_{u}\right) \\
& =\mathcal{C}\left(\nabla \pi_{c} \cdot \zeta_{u}\right)+\mathcal{C}\left(\nabla \phi_{t} \cdot \zeta_{u}\right) \\
& =\mathcal{C}\left(\nabla \pi_{c} \cdot \zeta_{u}\right)-2 \mathbb{E}\left(\phi_{t} \cdot \operatorname{div} \zeta_{u}\right) \\
& =\mathcal{C}\left(\nabla \pi_{c} \cdot \zeta_{u}\right) \\
& =I_{u}\left(\pi_{c}\right)
\end{aligned}
$$

\subsection{O Resultado Principal}

Estamos prontos agora para dar uma demonstração do Teorema 1.2.

\section{Demostração do Teorema 1.2:}

Considere a enumeração $S^{\mathrm{o}}=\left\{v_{n} ; n \in \mathbb{Z}\right\}$ dada em [16] pela qual $\tau_{v_{n}} \bar{S}^{\mathrm{o}}$ tem a mesma distribuição de $\bar{S}^{\text {o. }}$.

Queremos mostrar que existe $h_{c}: \Xi_{1} \rightarrow \mathbb{R}$, harmônica em $G\left(S^{\circ}\right)$, com gradiente covariante, e tal que para todo $n \in \mathbb{Z}$

$$
\lim _{t \rightarrow \infty} \mathbb{E}\left[\left|a\left(0, v_{n}, S^{\mathrm{o}}\right) \nabla \eta_{t}^{c}\left(0, v_{n}, \bar{S}^{\mathrm{o}}\right)-a\left(0, v_{n}, S^{\mathrm{o}}\right) \nabla h_{c}\left(0, v_{n}, \bar{S}^{\mathrm{o}}\right)\right|^{2}\right]=0
$$

Se existir $h_{c}$ que satisfaça o limite acima, então pela covariância de $\nabla \eta_{t}$ temos que

$$
\eta_{t}^{c}\left(v_{n}, v_{m}, \bar{S}^{\mathrm{o}}\right)=\eta_{t}^{c}\left(0, v_{m}-v_{n}, \tau_{v_{n}} \bar{S}^{\mathrm{o}}\right) \stackrel{L_{2}}{\longrightarrow} \nabla h_{c}\left(0, v_{m}-v_{n}, \tau_{v_{n}} \bar{S}^{\mathrm{o}}\right)
$$


e portanto $\nabla h_{c}$ é covariante.

Observe agora que

$$
\begin{aligned}
\mathbb{E}\left[\left|a\left(0, v_{n}, S^{\mathrm{o}}\right)\left[\nabla \eta_{t}^{c}\left(0, v_{n}, \bar{S}^{\mathrm{o}}\right)-\nabla h_{c}\left(0, v_{n}, \bar{S}^{\mathrm{o}}\right)\right]\right|^{2}\right] & \leq \mathbb{E}\left[\sum_{v \in V(0)}\left|\nabla \eta_{t}^{c}\left(0, v, \bar{S}^{\mathrm{o}}\right)-\nabla h_{c}\left(0, v, \bar{S}^{\mathrm{o}}\right)\right|^{2}\right] \\
& =2 \mathcal{C}\left(\left|\nabla \eta_{t}^{c}-\nabla h_{c}\right|^{2}\right) .
\end{aligned}
$$

Precisamos então mostrar que existe $h_{c}$ harmônica e tal que $\nabla \eta_{t}^{c}$ converge para $\nabla h_{c}$ na norma de $\mathcal{H}$. As ideias básicas para a prova são as seguintes: primeiro mostramos a existência de limites fracos por subsequências; em seguida mostramos que qualquer limite fraco tem divergente nulo; estudando as normas dos limites mostramos a unicidade e passamos de limite fraco para forte; verificando a propriedade do co-ciclo, mostramos que o limite é um gradiente; finalizamos mostrando que o limite possue inclinação correta nas direções coordenadas.

Dada uma função $f: \Xi_{1} \rightarrow \mathbb{R}$, também para simplificar, escreveremos apenas $f$ ao invés de $f\left(0, \bar{S}^{\mathrm{o}}\right)$.

Deste modo, como $\mathcal{C}\left(\left|\nabla \eta_{t}\right|^{2}\right)$ é decrescente (Corolário 4.4), segue que $\nabla \eta_{t}$ converge por subsequências na topologia fraca de $\mathcal{H}$, ou seja, para toda sequência $\left\{t_{k}\right\}_{k \geq 0}$, existe uma subsequência $\left\{t_{k_{j}}\right\}_{j \geq 0}$ e um campo $\zeta_{\infty} \in \mathcal{H}$ tais que

$$
\lim _{j \rightarrow \infty} \mathcal{C}\left(\nabla \eta_{t_{k_{j}}}^{c} \cdot \zeta\right)=\mathcal{C}\left(\zeta_{\infty} \cdot \zeta\right)
$$

para todo $\zeta \in \mathcal{H}$.

Considere agora um limite fraco $\zeta_{\infty}$ e a sequência $\left\{t_{k}\right\}_{k \geq 0}$ que leva a $\zeta_{\infty}$. Definindo $\phi=\frac{\operatorname{div} \zeta_{\infty}}{|V|}$, segue de (4.41), e da fórmula de integração por partes que

$$
\begin{aligned}
\frac{1}{2} \mathbb{E}\left[\frac{\left|\operatorname{div} \zeta_{\infty}\right|^{2}}{|V|}\right] & =\mathbb{E}\left[\phi \cdot \operatorname{div} \zeta_{\infty}\right] \\
& =-\mathcal{C}\left(\zeta_{\infty} \cdot \nabla \phi\right) \\
& =\lim _{k \rightarrow \infty}-\mathcal{C}\left(\nabla \eta_{t_{k}}^{c} \nabla \phi\right) \\
& =\lim _{k \rightarrow \infty} \mathbb{E}\left[\Delta \eta_{t_{k}}^{c} \cdot \phi\right] .
\end{aligned}
$$

Mas da desigualdade de Hölder, e do Lema 4.5 temos que

$$
\lim _{k \rightarrow \infty}\left|\mathbb{E}\left[\Delta \eta_{t_{k}}^{c} \cdot \phi\right]\right|^{2} \leq \lim _{k \rightarrow \infty} \mathbb{E}\left[\left|\frac{\Delta \eta_{t_{k}}^{c}}{|V(0)|}\right|^{2}\right] \mathbb{E}\left[\left|\operatorname{div} \zeta_{\infty}\right|^{2}\right]=0
$$


e portanto

$$
\operatorname{div} \zeta_{\infty}\left(0, \bar{S}^{\mathrm{o}}\right)=0
$$

quase certamente. E pela covariância de $\zeta_{\infty}$ (ver (4.40)), segue que para todo $n \in \mathbb{Z}$

$$
\operatorname{div} \zeta_{\infty}\left(v_{n}, \bar{S}^{\mathrm{o}}\right)=0
$$

quase certamente.

De (4.12), (4.41), (4.42), e da fórmula de integração por partes podemos concluir também que

$$
\begin{aligned}
\mathcal{C}\left(\left|\zeta_{\infty}\right|^{2}\right) & =\lim _{k \rightarrow \infty} \mathcal{C}\left(\nabla \eta_{t_{k}}^{c} \cdot \zeta_{\infty}\right) \\
& =\mathcal{C}\left(\nabla \pi_{c} \cdot \zeta_{\infty}\right)+\lim _{k \rightarrow \infty} \mathcal{C}\left(\nabla \phi_{t_{k}}^{c} \cdot \zeta_{\infty}\right) \\
& =\mathcal{C}\left(\nabla \pi_{c} \cdot \zeta_{\infty}\right)-\lim _{k \rightarrow \infty} \mathbb{E}\left[\phi_{t_{k}}^{c} \operatorname{div} \zeta_{\infty}\right] \\
& =\mathcal{C}\left(\nabla \pi_{c} \cdot \zeta_{\infty}\right) .
\end{aligned}
$$

Tome agora dois limites fracos $\zeta_{\infty, 1}$ e $\zeta_{\infty, 2}$. Usando (4.12), (4.41), (4.42), e a fórmula de integração por partes, segue que, se $\left(t_{k}\right)_{k>0}$ é a subsequência que leva para $\zeta_{\infty, 1}$, então

$$
\mathcal{C}\left(\zeta_{\infty, 1} \zeta_{\infty, 2}\right)=\lim _{k \rightarrow \infty} \mathcal{C}\left(\nabla \eta_{t_{k}}^{c} \zeta_{\infty, 2}\right)=\mathcal{C}\left(\nabla \pi_{c} \zeta_{\infty, 2}\right)+\lim _{k \rightarrow \infty} \mathcal{C}\left(\nabla \phi_{t_{k}} \zeta_{\infty, 2}\right)=\mathcal{C}\left(\left|\zeta_{\infty, 2}\right|^{2}\right)
$$

Usando o mesmo argumento para $\zeta_{\infty, 1}$

$$
\mathcal{C}\left(\left|\zeta_{\infty, 2}\right|^{2}\right)=\mathcal{C}\left(\left|\zeta_{\infty, 1}\right|^{2}\right)=\mathcal{C}\left(\zeta_{\infty, 1} \zeta_{\infty, 2}\right)
$$

e portanto

$$
\mathcal{C}\left(\left|\zeta_{\infty, 1}-\zeta_{\infty, 2}\right|^{2}\right)=0
$$

e o limite fraco é único.

Por outro lado

$$
\begin{aligned}
\mathcal{C}\left(\left|\nabla \eta_{t}\right|^{2}\right) & =\mathcal{C}\left(\nabla \pi_{c} \nabla \eta_{t}^{c}\right)+\mathcal{C}\left(\nabla \phi_{t} \nabla \eta_{t}\right) \\
& =\mathcal{C}\left(\nabla \pi_{c} \nabla \eta_{t}\right)-\mathbb{E}\left(\phi_{t} \Delta \eta_{t}^{c}\right)
\end{aligned}
$$

Da desigualdade de Hölder, segue que

$$
\left|\mathbb{E}\left[\phi_{t} \Delta \eta_{t}^{c}\right]\right|^{2} \leq \mathbb{E}\left[|V(0)|\left|\phi_{t}\right|^{2}\right] \mathbb{E}\left[\frac{\left|\Delta \eta_{t}^{c}\right|^{2}}{|V(0)|}\right] \leq \frac{E_{\nu_{o}}\left[E_{S^{\circ}}^{0}\left[\left|\left(c \cdot X_{t}\right)\right|^{2}\right]\right]}{t} t \mathbb{E}\left[\frac{\left|\Delta \eta_{t}^{c}\right|^{2}}{|V(0)|}\right] .
$$


Mas, pelo Lema $4.3, \mathbb{E}\left[\frac{\left|\Delta \eta_{t}\right|^{2}}{|V(0)|}\right]$ é integrável e portanto, existe uma sequência $\left(t_{k}\right)_{k \geq 0}$ tal que

$$
\lim _{k \rightarrow \infty} t_{k} \mathbb{E}\left[\frac{\left|\Delta \eta_{t_{k}}\right|^{2}}{|V(0)|}\right]=0
$$

Assim, do Lema 3.3, concluímos que

$$
\lim _{k \rightarrow \infty} \frac{E_{\nu_{o}}\left[E_{S^{\circ}}^{0}\left[\left|\left(c \cdot X_{t_{k}}\right)\right|^{2}\right]\right]}{t_{k}} t_{k} \mathbb{E}\left[\frac{\left|\Delta \eta_{t_{k}}^{c}\right|^{2}}{|V(0)|}\right]=0
$$

Deste modo, combinando (4.43), (4.45) e (4.46), segue que

$$
\mathcal{C}\left(\left|\nabla \eta_{t}^{c}\right|^{2}\right) \rightarrow \mathcal{C}\left(\left|\zeta_{\infty}\right|^{2}\right)
$$

e portanto $\nabla \eta_{t}^{c}$ converge forte (na norma de $\mathcal{H}$ ) para $\zeta_{\infty}$.

Em resumo, mostramos até agora que existe $\zeta_{\infty}: \Xi_{2} \rightarrow \mathbb{R}$ tal que div $\zeta_{\infty}=0$ quase-certamente, e

$$
\lim _{t \rightarrow \infty} \mathcal{C}\left(\left|\nabla \eta_{t}^{c}-\zeta_{\infty}\right|^{2}\right)=0
$$

Precisamos verificar agora que $\zeta_{\infty}$ é um campo gradiente. Isso é equivalente a mostrar que $\zeta_{\infty}$ satisfaz a propriedade do co-ciclo, ou seja, existe $\mathcal{N}^{\star} \subseteq \mathcal{N}^{m}, \operatorname{com} \mathbb{P}\left(\bar{S}^{\text {o }} \in \mathcal{N}^{\star}\right)=1$ e tal que para todo $\xi \in \mathcal{N}^{\star}$ e todo caminho $w_{0}, w_{1}, \ldots, w_{k}=w_{0} \in \xi \operatorname{com} a\left(w_{i}, w_{i-1}\right)=1, i=1, \ldots, k$

$$
\sum_{i=1}^{k} \zeta_{\infty}\left(w_{i}, w_{i-1}, \xi\right)=0
$$

Para isso, dados $i, j \in \mathbb{Z}$, observe que, por (4.40), $a\left(v_{i}, v_{j}\right) \nabla \eta_{t}\left(v_{i}, v_{j}\right)$ converge em $L_{2}$ para $a\left(v_{i}, v_{j}\right) \zeta_{\infty}\left(v_{i}, v_{j}\right)$. Existe portanto uma subsequência $\left(t_{k}\right)_{k \geq 0}$ de tempos para a qual a convergência é quase certa. Denote por $\mathcal{N}_{i, j} \subset \mathcal{N}^{m}$ o conjunto de medida um onde tal convergência ocorre.

Observe agora que, para todo $i, j \in \mathbb{Z}, a\left(v_{i}, v_{j}\right) \nabla \eta_{t_{k}}^{c}\left(v_{i}, v_{j}\right)$ converge quase certamente para $a\left(v_{i}, v_{j}\right) \zeta_{\infty}\left(v_{i}, v_{j}\right)$ ao longo da mesma subsequência $\left(t_{k}\right)_{k \geq 0}$. Defina então

$$
\mathcal{N}^{\star}=\bigcap_{i, j \in \mathbb{Z}} \mathcal{N}_{i, j}
$$

Basta agora notar que, como para cada $t \geq 0, \eta_{t}^{c}$ satisfaz a propriedade de co-ciclo, então $\zeta_{\infty}$ também deve satisfazer, e assim $\zeta_{\infty}$ tem que ser um gradiente.

Temos então que, para cada $c \in \mathbb{R}^{d}$, existe uma função harmônica $h_{c}$ tal que $\nabla \eta_{t}^{c}$ converge em $\mathcal{H}$ 
para $\nabla h_{c}$. Para finalizar, basta mostrar agora que, fixada uma direção coordenada $e$, a inclinação de $h_{c}$ é a quase certamente, a mesma de $\pi_{c}$.

Mas, do Lema 4.8 segue diretamente que

$$
\begin{aligned}
I_{e}\left(h_{c}\right) & =\mathcal{C}\left(\nabla h_{c} \cdot \zeta_{u}\right) \\
& =\lim _{t \rightarrow \infty} \mathcal{C}\left(\nabla \eta_{t}^{c} \cdot \zeta_{u}\right) \\
& =I_{e}\left(\pi_{c}\right),
\end{aligned}
$$

o que finaliza a demostração.

Vamos agora nos concentrar no caso bi-dimensional $(d=2)$. Em [4], Berger e Biskup trazem uma maneira construtiva de mostrar que o máximo da diferença entre a função harmônica e a função linear correspondente em uma caixa de raio $n$, é sub-linear em $n$. A idéia consiste em encontrar uma densidade positiva de pontos "bons", formando o que chamaremos de linhas "boas", ao longo das quais a sub-linearidade acontece com a mesma velocidade. Depois, controlando a distância entre tais linhas e usando o princípio do máximo para funções harmônicas, é possível controlar o máximo em caixas suficientemente grandes. A seguir adaptaremos tal técnica para o nosso caso. Dadas as características no processo que estamos trabalhando, algumas adaptações serão necessárias, mas as idéias centrais permanecem iguais.

Antes de passarmos à demonstração, faremos algumas considerações.

Inicialmente, dada uma direção coordenada $e \in \mathbb{R}^{2}$, defina

$$
Z_{k}:=\max _{T \in[k, k+1]}\{\|\operatorname{Cen}(T e, S)-T e\|\}
$$

e observe que, para $r>1$

$$
\mathbb{P}\left(Z_{k}>r\right)=\mathbb{P}\left(Z_{0}>r\right) \leq e^{-2 r}
$$

isto porque $\left(Z_{k}\right)$ é uma sequência estacionária e

$$
\left\{\max _{T \in[0,1]}\left\{\left\|\operatorname{Cen}\left(T e_{1}, S\right)-T e_{1}\right\|\right\}\right\} \subseteq\{S \cap[0,1] \times[-r+1, r-1]=\emptyset\} .
$$

De onde concluímos que $\mathbb{E}\left[\left|Z_{k}\right|^{n}\right]<\infty$ para todo $n$ e assim

$$
\lim _{k \rightarrow \infty} \frac{Z_{k}}{k}=0, \quad \text { q.c }
$$


$\mathrm{e}$

$$
\lim _{T \rightarrow \infty} \frac{\|\operatorname{Cen}(T e, S)-T e\|}{T}=0 \quad \text { q.c. }
$$

Dando sequência, queremos mostrar que, dado um plano $\pi_{c}(v)=(c \cdot v)$ e uma função harmônica $h$, tal que $I_{e}(h)=I_{e}\left(\pi_{c}\right)$ em qualquer direção coordenada $e \in \mathbb{R}^{2}$, então

$$
\lim _{n \rightarrow \infty} \max _{v \in[-n, n]^{2} \cap S^{\circ}}\left\{\frac{\left|h\left(v, S^{\mathrm{o}}\right)-\pi_{c}(v)\right|}{n}\right\}=0, \quad \text { q.c.. }
$$

Mas o evento $\Gamma=\{\xi \in \mathcal{N}:(4.49)$ acontece $\}$ é um evento claramente invariante por translações, e portanto, pela Proposição 2.9 , se $\mu(\Gamma)=1$ então $\mu_{\mathrm{o}}\left(\Gamma_{\mathrm{o}}\right)=1$, de modo que podemos deixar de lado o ponto na origem e trabahar apenas com o processo estacionário $S$.

Para simplificar a notação, a não ser que se faça necessário, deixaremos de lado a dependência em $S$ nas funções utilizadas. Utilizaremos também $e_{1}=(1,0)$ e $e_{2}=(0,1) \in \mathbb{Z}^{2}$.

Defina então $\psi(v, S)=h(v, S)-\pi_{c}(v)$ e, para cada $z \in \mathbb{Z}^{2}$ faça $v_{z}=\operatorname{Cen}(z, S)$. Agora, para $K>0$ e $\varepsilon>0$, diremos que $z \in \mathbb{Z}^{2}$ é $K, \varepsilon-$ bom se, para todo $T \in \mathbb{R}$

$$
\left|\psi\left(v_{z+T e}\right)-\psi\left(v_{z}\right)\right| \leq K+\varepsilon T
$$

$\mathrm{e}$

$$
\left|\pi_{c}\left(v_{z+T e}-(z+T e)\right)\right| \leq K+\varepsilon T .
$$

onde $e$ é um dos vetores coordenados.

Denote por $\mathbb{B}_{K, \varepsilon}(S)$ o conjunto dos sítios $K, \varepsilon-$ bom em $\mathbb{Z}^{2}$ para a configuração de pontos $S$.

Pela definição de inclinação dada em (1.10), se $I_{e_{1}}(\psi)=I_{e_{2}}(\psi)=0$, então existe $K_{1}>0$ suficientemente grande, tal que (4.50) seja satisfeito para a origem com probabilidade positiva. Usando agora (4.48), a mesma análise pode ser feita para (4.51). Deste modo, como $\mathbb{B}_{K, \varepsilon}$ é crescente em $K$, existe $K_{0}>0$ tal que $\mathbb{P}\left(0 \in \mathbb{B}_{K, \varepsilon}\right)>0$ para todo $K>K_{0}$.

Defina agora $X_{0}=\min \left\{k>0: k e_{1} \in \mathbb{B}_{K, \varepsilon}\right\}$. Para $i>0$ faça

$$
X_{i}:=\min \left\{k>X_{i-1}: k e_{1} \in \mathbb{B}_{K, \varepsilon}\right\},
$$

e para $i<0$

$$
X_{i}=\max \left\{k<X_{i+1}: k e_{1} \in \mathbb{B}_{K, \varepsilon}\right\} .
$$

Analogamente, defina $\left(Y_{i}\right)_{i \in \mathbb{Z}}$, usando o vetor $e_{2}$ no lugar de $e_{1}$. 
Lema 4.9. Se $K>K_{0}$ então

$$
\lim _{n \rightarrow \infty} \frac{\max \left\{\left(X_{i}-X_{i-1}\right): X_{i}, X_{i-1} \in[-n, n]\right\}}{n}=0, \quad \text { q.c., }
$$

$e$

$$
\lim _{n \rightarrow \infty} \frac{\max \left\{\left(Y_{i}-Y_{i-1}\right): Y_{i}, Y_{i-1} \in[-n, n]\right\}}{n}=0, \quad \text { q.c.. }
$$

Demonstração. Defina

$$
U_{z}=\left\{z \in \mathbb{B}_{K, \varepsilon}\right\},
$$

e note que

$$
\mathbf{1}_{U_{z}}(S)=\mathbf{1}_{U_{0}}\left(\tau_{z} S\right)
$$

Pelo Teorema Ergódico de Birkhoff vale então que

$$
\lim _{n \rightarrow \infty} \frac{1}{2 n+1} \sum_{k=-n}^{n} \mathbf{1}_{U_{k e_{1}}}(S)=\mathbb{P}\left(U_{0}\right), \quad \text { q.c. }
$$

Mas se (4.52) não ocorresse, o limite acima não poderia existir.

O mesmo argumento vale para justificar (4.53).

Passemos agora para a demonstração do Teorema 1.3.

Demonstração do Teorema 1.3. Tome $\mathcal{N}^{\sharp}$ o conjunto das configurações $\xi \in \mathcal{N}$ tais que (4.52) e (4.53) valham simultaneamente.

Fixe $\xi \in \mathcal{N}^{\sharp}$, e $\epsilon \in(0,1 / 2)$. Como $\mathbb{B}_{K, \varepsilon}$ é crescente em $K$, e $\cup_{K} \mathbb{B}_{K, \varepsilon}=\mathbb{Z}^{2}$, então podemos escolher $K=K(\xi)$, tal que $0 \in \mathbb{B}_{K, \varepsilon}$. Tome $n_{1}:=n_{1}(\xi)$ e $n_{2}:=n_{2}(\xi)$ tais que para todo $n>n_{1}$

$$
\max \left\{\left(X_{i}-X_{i-1}\right): X_{i}, X_{i-1} \in[-n, n]\right\} \leq \varepsilon n,
$$

e para todo $n>n_{2}$

$$
\max \left\{\left(Y_{i}-Y_{i-1}\right): Y_{i}, Y_{i-1} \in[-n, n]\right\} \leq \varepsilon n .
$$

Para cada $i \in \mathbb{Z}$, defina retas horizontais

$$
l_{i}^{1}=\left\{Y_{i}+t(1,0): t \in \mathbb{R}\right\}
$$

e retas verticais

$$
l_{i}^{2}=\left\{X_{i}+t(0,1): t \in \mathbb{R}\right\}
$$


Defina agora o grid $\mathbb{G}=\bigcup_{i \in \mathbb{Z}}\left\{l_{i}^{1} \cup l_{i}^{2}\right\}$

Fixe $n>\max \left\{n_{1}, n_{2}\right\}$ e tome $v \in \xi \cap[-n, n]^{2}$. Suponha agora que $v$ esteja entre as linhas verticais $l_{i}^{2}$ e $l_{i+1}^{2}$ de $\mathbb{G}$. Vamos agora tentar localizar estas linhas. Observe primeiro que, como $n>n_{1}$, então $\left|X_{i}\right| \leq n$ ou $\left|X_{i+1}\right| \leq n$. Suponha então que $\left|X_{i}\right| \leq n$. Queremos mostrar que $\left|X_{i+1}\right| \leq 2 n$. Mas se $X_{i+1}>n$, então em particular, $X_{i+1}>n_{1}$ e portanto

$$
X_{i+1}-X_{i} \leq \max \left\{\left(X_{j}-X_{j-1}\right):\left|X_{j}\right|<X_{i+1},\left|X_{j-1}\right|<X_{i+1}\right\} \leq \varepsilon X_{i+1} .
$$

De onde segue que

$$
X_{i+1} \leq \frac{n}{1-\varepsilon} \leq 2 n
$$

Podemos concluir ainda que

$$
X_{i+1}-X_{i} \leq 2 \varepsilon n
$$

Aplicando o mesmo argumento para as linhas horizontais, concluímos que se $v \in S \cap[-n, n]^{2}$ então $v$ pertence a um retângulo inteiramente contido dentro de $[-2 n, 2 n]^{2}$, cuja fronteira é formada por pedaços de linhas em $\mathbb{G}$, e cuja distância entre faces paralelas não ultrapassa $2 \varepsilon n$.

Suponha agora que $\mathrm{C}(v) \cap \mathbb{G} \neq \emptyset$. Sem perda de generalidade, suponha que $\mathrm{C}(v)$ seja cruzada por uma linha horizontal, digamos $l_{i}^{x}$. Isso significa que existe $\alpha \in[-2 n, 2 n]$ tal que $v=\operatorname{Cen}\left(Y_{i}+\alpha e_{1}\right)$, e portanto por (4.50)

$$
|\psi(v)| \leq 2 K+\left(\alpha+Y_{i}\right) \varepsilon \leq 2 K+4 \varepsilon n .
$$

Se $\mathrm{C}(v) \cap \mathbb{G}=\emptyset$, então procedemos da seguinte maneira. Denote por $R_{v}$ o retângulo com fronteira no grid $\mathbb{G}$ envolvendo $v$. Denote agora por $\mathcal{G}_{v}$ o subgrafo de $\mathcal{G}(\xi)$ formado apenas pelos pontos $w \in \xi$ tais que $\mathrm{C}(w) \cap R_{v} \neq \emptyset$. Note que se $w \in \xi$ é vizinho de $v$, então $w \in \mathcal{G}_{v}$, e que a fronteira $\delta \mathcal{G}_{v}$ de $\mathcal{G}_{v}$ é composta apenas por pontos cuja célula de Voronoi é cortada pela fronteira de $R_{v}$. Observe agora que $x \mapsto h(x)=\psi(x)+\pi_{c}(x)$ é harmônica em $\mathcal{G}_{v}$ com condições de fronteira dadas por $\left.h\right|_{\delta \mathcal{G}_{v}}$, e portanto, pelo Princípio do máximo e do mínimo para funções harmônica, existem $v^{\prime}, v^{\prime \prime} \in \delta \mathcal{G}_{v}$ tais que $h\left(v^{\prime}\right) \leq h(v) \leq h\left(v^{\prime \prime}\right)$, e assim podemos encontrar $v^{*} \in \delta G_{v}$ tal que

$$
|\psi(v)| \leq\left|\psi\left(v^{*}\right)-\pi_{c}(v)\right| \leq\left|\psi\left(v^{*}\right)\right|+\left|\pi\left(v^{*}-v\right)\right|
$$

Por (4.54), $\left|\psi\left(v^{*}\right)\right| \leq 2 K+4 \varepsilon n$. Por outro lado, podemos repetir a argumentação usada na conclusão de (4.54) e supor que $v^{*}=\operatorname{Cen}\left(Y_{i}+\alpha e_{1}\right)$ para algum $i$ e $\alpha \in[-2 n, 2 n]$. Assim, usando (4.51), $v \in \mathcal{G}_{v}$ e o fato dos lados de $R_{v}$ estarem a uma distância menos que $2 \varepsilon n$, concluímos que

$$
\left|\pi_{c}\left(v^{*}-v\right)\right| \leq\left|\pi_{c}\left(v^{*}-\left(Y_{i}+\alpha e_{1}\right)\right)\right|+\left|\pi_{c}\left(Y_{i}+\alpha e_{1}-v\right)\right| \leq K+4 \varepsilon n,
$$


e portanto

$$
\|\psi(v)\| \leq 3 K+8 \varepsilon n,
$$

o que conclui a demonstração. 


\section{Capítulo 5}

\section{Considerações Finais}

\subsection{Aplicação - O Princípio de Invariância}

Vamos agora utilizar os resultados apresentados para $d=2$, e demonstrar o princípio de invariância para o passeio aleatório simples na triangulação de Delaunay, introduzido no capítulo 3.

A principal idéia por trás da demostração deste resultado é a de utilizar a deformação harmônica definida na seção 1.1.3 para transformar o passeio aleatório em um martingal. O princípio de invariância para este novo passeio segue do TCL Funcional para Martingais (Teorema 7.4 de [9]), depois de verificadas algumas condições. A partir daí, utiliza-se a sub-linearidade da deformação (Teorema 1.3) para mostrar que, na mesma escala, a diferença dos dois passeios converge a zero.

Tais idéias são as mesmas que permeiam os resultados apresentados em [4] e [20], no caso do cluster de percolação supecrítico. Infelizmente, para $d \geq 3$, a sublinearidade em direções coordenadas, dada no Teorema 1.2, não é o bastante para controlar a diferença. Nos artigos citados acima, os autores precisam lançar mão das estimativas para as probabilidades de transição em $n$ passos do passeio aleatório no cluster, calculados por Barlow em [2], e ainda não determinadas para o nosso caso.

Antes de passarmos para o enunciado e demostração do resultado, vamos fixar algumas notações. Seja $\tilde{X}_{n}$ o passeio aleatório em tempo discreto definido na Seção 3.2. Para $\varepsilon>0$ seja

$$
X_{t}^{n}=\frac{1}{\sqrt{n}}\left[\tilde{X}_{\lfloor t n\rfloor}+(t n-\lfloor t n\rfloor)\left(\tilde{X}_{\lfloor t n\rfloor+1}-\tilde{X}_{\lfloor t n\rfloor}\right)\right] .
$$

Considere agora as funções harmônicas $h_{1}, h_{2}$ determinadas pelo Teorema 1.2, e defina

$$
h(\cdot, \cdot)=\left(h_{1}(\cdot, \cdot), h_{2}(\cdot, \cdot)\right)
$$


Abusando da notação, escreveremos

$$
\mathcal{C}\left(|\nabla h|^{2}\right)=\mathcal{C}\left(\left|\nabla h_{1}\right|^{2}\right)+\mathcal{C}\left(\left|\nabla h_{2}\right|^{2}\right)
$$

Denote por $\theta: \mathbb{R}^{2} \rightarrow \mathbb{R}^{2}$ o operador de rotação de $\frac{\pi}{2}$ em torno da origem, e considere sua extensão para $\mathcal{N}^{m}$ dada por

$$
\theta \bar{\xi}=\left\{\left(\theta(v), m_{v}\right):\left(v, m_{v}\right) \in \bar{\xi}\right\}
$$

Como temos feito desde o início deste trabalho, denotaremos por $S^{\text {o }}$ o processo de Poisson em $\mathbb{R}^{d}, S^{\mathrm{o}}=S+\delta_{0}$ sua versão de Palm com lei $\mu_{\mathrm{o}}$. Observe que pela construção da dinâmica, e pela isotropia do processo de Poisson

$$
\eta_{t}^{e_{1}}\left(v, \bar{S}^{\mathrm{o}}\right)=\eta_{t}^{e_{2}}\left(\theta(v), \theta \bar{S}^{\mathrm{o}}\right) \quad \text { e } \quad \eta_{t}^{e_{2}}\left(v, S^{\mathrm{o}}\right)=-\eta_{t}^{e_{1}}\left(\theta(v), \theta S^{\mathrm{o}}\right)
$$

e portanto

$$
\mathcal{C}\left(\left|\nabla h_{2}\right|^{2}\right)=\lim _{t \rightarrow \infty} \mathcal{C}\left(\left|\nabla \eta_{t}^{e_{1}}\right|^{2}\right)=\lim _{t \rightarrow \infty} \mathcal{C}\left(\left|\nabla \eta_{t}^{e_{2}}\right|^{2}\right)=\mathcal{C}\left(\left|\nabla h_{1}\right|^{2}\right),
$$

e assim

$$
\mathcal{C}\left(|\nabla h|^{2}\right)=2 \mathcal{C}\left(\left|\nabla h_{1}\right|^{2}\right)
$$

Além disso, seguindo os mesmos argumentos que em (4.44), obtemos que

$$
\mathcal{C}\left(\nabla h_{1} \nabla h_{2}\right)=\lim _{t \rightarrow \infty} \mathcal{C}\left(\nabla \pi_{e_{1}} \nabla \eta_{t}^{e_{2}}\right)=\lim _{t \rightarrow \infty} \mathcal{C}\left(\nabla \pi_{e_{2}} \nabla \eta_{t}^{e_{1}}\right)
$$

Mas por (5.1) e pela isotropia de $\bar{S}^{\text {o, }}$

$$
\mathcal{C}\left(\nabla \pi_{e_{1}} \nabla \eta_{t}^{e_{2}}\right)=-\mathcal{C}\left(\nabla \pi_{e_{2}} \nabla \eta_{t}^{e_{1}}\right)
$$

e portanto

$$
\mathcal{C}\left(\nabla h_{1} \nabla h_{2}\right)=0
$$

Dado $T>0$, denote por $\left(C[0, T], \mathcal{B}_{T}\right)$ a conjunto das funções contínuas de $[0, T]$ em $\mathbb{R}$, munido com a $\sigma$-álgebra de Borel relativa a norma do supremo.

Com a notação fixada acima mostraremos agora o seguinte resultado.

Teorema 5.1. Considere $d=2$. Dado $\xi \in \mathcal{N}^{\circ}$ seja $\left\{\tilde{X}_{n} ; n \geq 0\right\}$ o passeio aleatório com lei $P_{\xi}^{0}$. Então, $\mu_{\mathrm{o}}$-quase certamente, e para todo $T>0$, a lei do processo $\left(X_{t}^{n} ; t \in[0, T]\right)$ em $\left(C[0, T], \mathcal{B}_{T}\right)$ converge fraco quando $n \rightarrow \infty$, para a lei de um movimento Browniano isotrópico $\left(B_{t} ; t \in[0, T]\right)$ em $\mathbb{R}^{2}$, com constante de difusão $D=E\left(\left|B_{1}\right|^{2}\right)=\frac{1}{\mathbb{E}[|V(0)|]} \mathcal{C}\left(|\nabla h|^{2}\right)$. 
Demonstração. Defina

$$
M_{n}=h\left(\tilde{X}_{n}\right),
$$

e faça $\mathcal{F}_{n}=\sigma\left(\tilde{X}_{1}, \ldots, \tilde{X}_{n}\right)$. Como, para $\mu_{\mathrm{o}}$-quase todo $\xi \in \mathcal{N}^{\circ}$, a função $h: v \in \xi \rightarrow h(v, \xi)$ é harmônica em $\mathcal{G}(\xi)$, então

$$
E_{\xi}^{0}\left[M_{n+1} \mid \mathcal{F}_{n}\right]=\frac{1}{\left|V\left(\tilde{X}_{n}\right)\right|} \sum_{v \in V\left(\tilde{X}_{n}\right)} h(v)=h\left(\tilde{X}_{n}\right)=M_{n},
$$

e, portanto, $\left(M_{n}\right)_{n \geq 0}$ é um martingal com respeito a $\left\{\mathcal{F}_{n} ; n \geq 0\right\}$. Além disso, segue diretamente do fato de $\nabla h_{1}, \nabla h_{2} \in \mathcal{H}$ que $M_{n}$ é quadrado-integrável.

Denotando por $M_{t}^{n}$ a interpolação linear de $n^{-\frac{1}{2}} M_{\lfloor t n\rfloor}$, vamos mostrar que a lei de $\left(M_{t}^{n} ; t \in[0, T]\right)$ converge fracamente, quando $n \rightarrow \infty$, para a lei de $\left(B_{t} ; t \in[0, T]\right)$. Para isso mostraremos primeiro que para todo $c \in \mathbb{R}^{2}$, a lei do processo $\left(c \cdot M_{t}^{n} ; t \in[0, T]\right)$ converge para a lei de um movimento Browniano unidimensional com coeficiente de difusão $\frac{1}{2} D\|c\|^{2}$.

Pelo TCL Funcional para Martingais (ver [9]) precisamos mostrar que, $\mu_{\mathrm{o}}$-quase certamente,

$$
V_{n}^{\epsilon}:=\frac{1}{n} \sum_{k=1}^{n} E_{\xi}^{0}\left[\left(c \cdot\left(M_{k+1}-M_{k}\right)\right)^{2} \mathbf{1}_{\left\{\left|c \cdot\left(M_{k+1}-M_{k}\right)\right| \geq \epsilon \sqrt{n}\right\}} \mid \mathcal{F}_{k}\right] \stackrel{n \rightarrow \infty}{\longrightarrow} 0
$$

para todo $\epsilon>0$, e

$$
V_{n}:=\frac{1}{n} \sum_{k=1}^{n} E_{\xi}^{0}\left[\left(c \cdot\left(M_{k+1}-M_{k}\right)\right)^{2} \mid \mathcal{F}_{k}\right] \stackrel{n \rightarrow \infty}{\longrightarrow} \frac{1}{2} D\|c\|^{2} .
$$

Para isso, observe que

$$
\left(c \cdot\left(M_{k+1}-M_{k}\right)\right)=\left(c \cdot\left(h\left(\tilde{X}_{k+1}, \xi\right)-h\left(\tilde{X}_{k}, \xi\right)\right)\right)=\left(c \cdot h\left(\tilde{X}_{k+1}-\tilde{X}_{k}, \tau_{\tilde{X}_{k}} \xi\right)\right.
$$

Portanto, se definirmos

$$
\phi_{N}(\xi)=E_{\xi}^{0}\left[\left(c \cdot M_{1}\right)^{2} \mathbf{1}_{\left|\left(c \cdot M_{1}\right)\right| \geq N}\right]
$$

temos que

$$
V_{n}^{\epsilon}=\frac{1}{n} \sum_{k=1}^{m} \phi_{\epsilon \sqrt{n}}\left(\tau_{\tilde{X}_{k}} \xi\right)
$$

$\mathrm{e}$

$$
V_{n}=\frac{1}{n} \sum_{k=1}^{m} \phi_{0}\left(\tau_{\tilde{X}_{k}} \xi\right)
$$


Fixado $N>0$, tomando $n$ suficientemente grande, temos

$$
\phi_{\epsilon \sqrt{n}}(\xi) \leq \phi_{N}(\xi)
$$

e portanto, para todo $\epsilon>0$, segue do Lema 3.1, e do Teorema Ergódico de Birkhoff, que

$$
\limsup _{n \rightarrow \infty} V_{n}^{\epsilon} \leq E_{\nu_{o}}\left[E_{\xi}\left[\left|\left(c \cdot\left(M_{1}\right)\right)\right|^{2} \mathbf{1}_{\left|M_{1}\right| \geq N}\right]\right]
$$

Assim, fazendo $N \rightarrow \infty$, pelo Teorema da Convergência Dominada

$$
\lim _{n \rightarrow \infty} V_{n}^{\epsilon}=0
$$

Do mesmo modo

$$
\lim _{n \rightarrow \infty} V_{n}=E_{\nu_{o}}\left[E_{\xi}\left[\left|\left(c \cdot\left(M_{1}\right)\right)\right|^{2}\right]\right] .
$$

Mas por (5.2) e (5.3) obtemos que

$$
E_{\nu_{o}}\left[E_{\xi}\left[\left|\left(c \cdot\left(M_{1}\right)\right)\right|^{2}\right]\right]=\frac{1}{2}[\mathbb{E}[|V(0)|]]^{-1} \mathcal{C}\left(|\nabla h|^{2}\right)\|c\|^{2}=\frac{1}{2} D\|c\|^{2} .
$$

Concluimos então que $\left(c \cdot M_{t}^{n} ; t \in[0, T]\right)$ converge para um movimento Browniano com coeficiente de difusão

$$
\frac{1}{2} D\|c\|^{2}
$$

com $D=[\mathbb{E}[|V(0)|]]^{-1} \mathcal{C}\left(|\nabla h|^{2}\right)>0$. Agora, pelo dispositivo de Cramér-Wold (ver [9]), segue que a lei de $\left(M_{t}^{n} ; t \in[0, T]\right)$ converge fraco para a lei de $\left(B_{t} ; t \in[0, T]\right)$.

Para finalizar a demostração precisamos mostrar que $\left(X_{t}^{n}-M_{t}^{n} ; t \in[0, T]\right)$ converge fraco a zero, quando $n \rightarrow \infty$. Para isso, considerando $T=1$, é suficiente mostrar que, $\mu_{\mathrm{o}}$-quase certamente,

$$
\max _{0 \leq k \leq n} \frac{1}{\sqrt{n}}\left\|\tilde{X}_{k}-M_{k}\right\| \stackrel{n \rightarrow \infty}{\longrightarrow} 0
$$

em $P_{\xi}^{0}$-probabilidade.

Neste sentido, observe primeiro que pelo Teorema 1.3, dado $\epsilon>0$ existe $K>0$, dependendo de $\epsilon$ e do meio $\xi$, tal que para todo $v \in \xi$

$$
\|\psi(v)\|=\|h(v)-v\| \leq K+\epsilon\|v\|
$$


Tomando $\varepsilon<\frac{1}{2}$ temos que

$$
\|\psi(v)\| \leq 2 K+2 \epsilon\|h(v)\|
$$

e portanto, pelo Teorema de Portmanteau

$$
\begin{aligned}
\limsup _{n \rightarrow \infty} P_{\xi}^{0}\left\{\max _{0 \leq k \leq n}\left\|\psi\left(\tilde{X}_{k}\right)\right\| \geq \delta \sqrt{n}\right\} & \leq \limsup _{n \rightarrow \infty} P_{\xi}^{0}\left\{\max _{0 \leq k \leq n}\left\|M_{k}\right\| \geq \frac{\delta \sqrt{n}}{2 \epsilon}-K \epsilon^{-1}\right\} \\
& \leq P\left\{\max _{t \in[0,1]}\left|B_{t}\right| \geq \frac{\delta}{2 \epsilon}\right\}
\end{aligned}
$$

e o último termo da expressão acima converge a zero quando $\epsilon \rightarrow 0$.

\subsection{Problemas Abertos}

Para finalizar vamos comentar rapidamente alguns dos problemas que continuam sem solução, e que pretendemos abordar no futuro.

Antes de mais nada, gostariamos de mostrar a convergência quase-certa da nossa dinâmica. Nossa prova para convergência não determina a taxa de decaimento da distância $L_{2}$, o que ajudaria a determinar tal convergência.

Outro resultado que ainda não temos é o da unicidade (módulo somas) da função harmônica com gradiente covariante e inclinação pré-definida. Este mesmo problema, considerado no caso do cluster de percolação, é também deixado como aberto nas considerações finais de [4] e, como os autores comentam, ainda não está claro que condições são necessárias para haver unicidade da função harmônica com comportamento assintótico linear pré-definido.

Outro problema interessante seria encontrar uma demonstração para o Teorema 1.3 para o caso $d>2$, o que permitiria concluir o princípio de invariância também nestas dimensões.

Para terminar este trabalho, vamos descrever em poucas palavras um problema similar ao estudado aqui, e que achamos igualmente interessante.

Fixe uma caixa $A_{n}:=[-n, n]^{d}$ e tome $\mathcal{G}_{n}=\left(\mathcal{V}_{n}, \mathcal{E}_{n}\right)$ a triangulação de Delaunay restrita a $A_{n}$. Mais exatamente, faça

$$
\mathcal{V}_{n}=\bigcup_{v \in S^{\circ} \cap A_{n}} V(v)
$$

e $\mathcal{E}_{n}$ todos os elos de $\mathcal{E}$ cujo vértice inicial está em $A_{n}$. Chamaremos de fronteira os vértices de $\partial \mathcal{V}_{n}:=\mathcal{V}_{n} \backslash\left\{S^{\circ} \cap A_{n}\right\}$. 
Fixando condições de fronteira apropriadas, como por exemplo

$$
f_{n}(v)=v_{1}, \quad v \in \partial \mathcal{V}_{n},
$$

ou ainda

$$
f_{n}(v)= \begin{cases}n & , \quad \text { se } v \in \partial \mathcal{V}_{n} \text { e } v_{1}>0 \\ -n & , \quad \text { se } v \in \partial \mathcal{V}_{n} \text { e } v_{1}<0\end{cases}
$$

denote por $\phi_{n}$ a única função harmônica em $\mathcal{V}_{n}$ e condições de fronteira dadas por $f_{n}$. Gostaríamos de saber se, de algum modo, a sequência $\left(\phi_{n}\right)_{n>0}$ se aproxima de alguma função harmônica em todo grafo. Seria interessante se, além disto, a função encontrada fosse a mesma determinada pelo processo de Harness, mas até o momento não temos claro nem se o limite, caso exista, possui gradiente covariante. 


\section{Apêndice A}

\section{Resultados Auxiliares}

\section{A.1 Funções Harmônicas em Grafos Finitos}

Seja $\mathcal{G}=(\mathcal{V}, \mathcal{E})$ um grafo finito conexo. Escreva $\mathcal{V}=I \cup B$ de modo que

(a) $I \cap B=\emptyset$;

(b) Todo vértice em $\mathcal{V}$ possui ao menos um vizinho em $I$;

(c) O subgrafo $\mathcal{G}^{\prime}=\left(I, \mathcal{E}^{\prime}\right)$ de $\mathcal{G} \operatorname{com} \mathcal{E}^{\prime}=\{(u, v) \in \mathcal{E}: u, v \in I\}$ é conexo.

Denote $I$ de vértices interiores e $B$ de fronteira de do grafo $\mathcal{G}$. Por uma questão de coerência, consideraremos que $\mathcal{G}$ é orientado, mas que $(u, v) \in \mathcal{E}$ se e só se $(v, u) \in \mathcal{E}$.

A seguir repetiremos algumas das definições já colocadas na introdução do trabalho. Considere então um conjunto de pesos (condutividade) $(a(u, v))_{(u, v \in \mathcal{E})}$ positivos e tais que $a(u, v)=a(v, u)$. Para facilitar a notação estenda os pesos $a(\cdot, \cdot)$ para todo $\mathcal{V} \times \mathcal{V}$ fazendo $a(u, v)=0$ se $(u, v) \notin \mathcal{E}$, e faça $a_{u}=\sum_{v \in \mathcal{V}} a(u, v)$. Defina também a matriz $P:=(p(u, v))_{(u, v) \in \mathcal{E}}$ dada por

$$
p(u, v)=\frac{a(u, v)}{a_{u}}
$$

Note que, pela conectividade de $G$ a matriz $P$ define uma cadeia de Markov irredutível em $V$ e que a medida de probabilidade dada por $\mu(u)=\frac{a_{u}}{\sum_{v} a_{v}}$, é reversível para $P$. De fato, se $c=\sum_{v} a_{v}$ então

$$
\mu(u) p(u, v)=c a_{u} p(u, v)=c a(u, v)=c a(v, u)=c a_{v} p(v, u)=\mu(v) p(v, u) .
$$


Diremos portanto que uma função $h: \mathcal{V} \rightarrow \mathbb{R}$ é harmônica para $P:=(p(v, w))_{v, w \in \mathcal{V}}$ em $I$ se

$$
h(v)=\sum_{w \in \mathcal{V}} p(v, w) h(w), \text { para todo } v \in I .
$$

Observe que (A.1) é o mesmo que

$$
\sum_{w \in \mathcal{V}} a(v, w)(h(v)-h(w))=0, \text { para todo } v \in I
$$

No caso em que $I=\mathcal{V}$ diremos simplesmente que $h$ é harmônica em $\mathcal{G}$.

Para verificar a existência de tais funções vamos considerar o seguinte exemplo: Seja $\left(X_{n}\right)_{n \geq 0}$ uma cadeia de markov com probabilidade de transição dada por $P$. Considere o tempo de parada $T=\min \left\{n \geq 0: X_{n} \in B\right\}$ e note que, como $V$ é finito e $X_{n}$ é irredutível, então $T<\infty$ quase certamente. Considere agora uma função $f: B \rightarrow \mathbb{R}$ definida na fronteira de $G$, e defina a função $h: V \rightarrow \mathbb{R}$ por

$$
h(u)=\mathbb{E}\left[f\left(X_{T}\right) \mid X_{0}=u\right] .
$$

Observe agora que se $u \in B$ então $T=0$ e portanto $h(u)=f(u)$. Agora, para $u \in I$ vale que

$$
h(u)=\sum_{v \in V} \mathbb{E}\left[f\left(X_{T}\right) \mid X_{1}=v\right] \mathbb{P}\left(X_{1}=v \mid X_{0}=u\right)=\sum_{v \in V} h(v) p(u, v)
$$

e portanto $h$ é harmônica em $I$ com condição de fronteira dada por $f$.

Um resultado importante para grafos finitos é o chamado Princípio do Máximo e Mínimo que diz que

Proposição A.1 (Princípio do Máximo e do Mínimo). Se $h: \mathcal{V} \rightarrow \mathbb{R}$ é harmônica para $P$ em I com condição de fronteira $f: B \rightarrow \mathbb{R}$ então os valores máximo e o mínimo de $h$ são atingidos em $B$, ou seja, existem vértices $v_{1}, v_{2} \in B$ tais que

$$
h\left(v_{1}\right)=\max _{u \in \mathcal{V}} h(u)
$$

$e$

$$
h\left(v_{2}\right)=\min _{u \in \mathcal{V}} h(u)
$$

Demonstração. Como $\mathcal{V}$ é finito, para mostrar (A.3) basta mostrar que para todo $u \in I$ existe $v \in B$ tal que $h(u) \leq h(v)$. Para isso considere $v \in I$. Se $h$ é constante o resultado segue imediatamente. Suponha então que $h$ não seja constante. 
Como $h(v)$ é uma média ponderada do valor de $h$ em seus vizinhos, então $h(v)$ deve ser estritamente menor ao máximo de $h$ nos vizinhos de $v$ (lembre-se que estamos supondo $h$ não constante). Seja $v_{1}$ este vizinho. Se $v_{1} \in B$ acabou, se $v_{1} \in I$ repetimos o mesmo argumento para encontrar $v_{2}$. Definimos assim uma sequência $v_{1}, v_{2}, \ldots$. Note que se $v_{n}$ permanecer em $I$ então deverão haver $i>j$ tais que $v_{i}=v_{j}$. Mas por construção $h\left(v_{n+1}\right)-h\left(v_{n}\right)>0$ e portanto

$$
0=h\left(v_{i}\right)-h\left(v_{j}\right)=\sum_{k=1}^{i-j} h\left(v_{j+k}\right)-h\left(v_{j+k-1}\right)>0 .
$$

Portanto teremos que, eventualmente $v_{n} \in B$.

A equação (A.4) sai seguindo os mesmos argumentos.

Como corolário segue que

Corolário A.2. Se $h: V \rightarrow \mathbb{R}$ é harmônica para $P$ em I com condições de fronteira $f: B \rightarrow \mathbb{R}$ constate $(f \equiv c)$ então h é também constante igual a $c$.

Corolário A.3. Se $h_{1}: V \rightarrow \mathbb{R}$ e $h_{2}: V \rightarrow \mathbb{R}$ são harmônicas para $P$ em I com condições de fronteira $f: B \rightarrow \mathbb{R}$ então $h_{1}=h_{2}$.

Demonstração. Basta notar que $h=h_{1}-h_{2}$ é harmônica com condição de fronteira constante e igual a 0 .

Outra característica importante das funções harmônicas é a de ser, dentre todas as funções definidas em $V$ com mesma condição de fronteira, a que minimiza um dado funcional conhecido como energia. Este resultado, conhecido no contexto de redes elétricas como Princípio de Thompson, e normalmente enunciado no contexto de fluxos unitários, pode ser encontrado em [8]. Abaixo enunciaremos uma versão um pouco mais geral, mas cuja demonstração é essencialmente a mesma.

Proposição A.4. Seja $h: V \rightarrow \mathbb{R}$ a (única) função harmônica para $P$ em I com condições de fronteira dadas por $f: B \rightarrow \mathbb{R}$. Se $g: V \rightarrow \mathbb{R}$ é tal que $g(u)=f(u)=h(u)$ para todo $u \in B$, então

$$
\sum_{u, v \in V}(h(u)-h(v))^{2} a(u, v) \leq \sum_{u, v \in V}(g(u)-g(v))^{2} a(u, v) .
$$


Demonstração. Defina $d:=g-h$, e observe que $d(u)=0$ para todo $u \in B$. Temos então que

$$
\begin{gathered}
\sum_{u, v \in V}(g(u)-g(v))^{2} a(u, v)=\sum_{u, v \in V}(h(u)-h(v))^{2} a(u, v)+\sum_{u, v \in V}(d(u)-d(v))^{2} a(u, v) \\
+2 \sum_{u, v \in V}(h(u)-h(v)) a(u, v)(d(u)-d(v)) .
\end{gathered}
$$

Como $h$ é harmônica em $I$, então $\sum_{v}(h(u)-h(v)) a(u, v)=0$ para todo $u \in I$ e assim

$$
\begin{aligned}
\sum_{u, v \in V}(h(u)-h(v)) a(u, v)(d(u)-d(v))= & \sum_{u \in V} d(u) \sum_{v \in V}(h(u)-h(v)) a(u, v) \\
& -\sum_{v \in V} d(v) \sum_{u \in V}(h(u)-h(v)) a(u, v) \\
= & \sum_{u \in I} d(u) \sum_{v \in V}(h(u)-h(v)) a(u, v) \\
& -\sum_{v \in I} d(v) \sum_{u \in V}(h(u)-h(v)) a(v, u) \\
= & 0 .
\end{aligned}
$$

E portanto

$$
\begin{aligned}
\sum_{u, v \in V}(g(u)-g(v))^{2} a(u, v) & =\sum_{u, v \in V}(h(u)-h(v))^{2} a(u, v)+\sum_{u, v \in V}(d(u)-d(v))^{2} a(u, v) \\
& \geq \sum_{u, v \in V}(h(u)-h(v))^{2} a(u, v) .
\end{aligned}
$$

Na teoria de redes elétricas, um campo $j: \mathcal{E} \rightarrow \mathbb{R}$ tal que $j(u, v)=-j(v, u)$ é conhecido como fluxo, e o funcional

$$
\mathbf{E}[j]:=\sum_{u, v \in V}(j(u, v))^{2} a(u, v)
$$

representa a energia dissipada no circuito pelo fluxo $j$. Para maiores detalhes sobre o assunto ver [8].

\section{A.2 A Medida de Campbell}

A seguir vamos justificar a existência da medida $\mathcal{C}$ definida em (4.17). As demonstrações dos resultados aqui apresentados podem ser encontradas em [7].

Dado o processo pontual marcado $\bar{S}^{\text {o }}$, e sendo $\mathcal{X}=\mathbb{R}^{d} \times \mathbb{R}^{d} \times \mathcal{N}^{m}$, podemos associar à lei de $\bar{S}^{\text {o }}$ 
uma medida $\hat{\mathcal{C}}$ definida em $(\mathcal{X}, \mathcal{B}(\mathcal{X}))$ como a medida que para cada $A, B \in \mathcal{B}\left(\mathbb{R}^{d}\right)$ e $U \in \mathcal{B}\left(\mathcal{N}^{m}\right)$ vale

$$
\hat{\mathcal{C}}(A \times B \times U)=\mathbb{E}\left[\left|S^{\mathrm{o}} \cap A\right|\left|S^{\mathrm{o}} \cap B\right| \mathbf{1}_{U}\left(\bar{S}^{\mathrm{o}}\right)\right] .
$$

Esta medida é conhecida como medida de Campbell de segunda ordem associada a $\bar{S}^{\text {o }}$. Sua caracterização é dada no lema abaixo, cuja demostração pode ser encontrada em [7]. As medidas de Campbell de primeira ordem estão diretamente relacionadas com as medidas de Palm. Em verdade, usando tais medidas é possível definir medidas de Palm em um contexto mais geral de medidas aleatórias localmente finitas (não necessariamente medidas de contagem), sem a necessidade de pedir estacionariedade.

Lema A.5. A medida de Campbell definida em (A.5) concentra massa em

$$
\Xi_{2}=\left\{(x, y, \bar{\xi}) \in \mathbb{R}^{d} \times \mathbb{R}^{d} \times \mathcal{N}^{m}: x, y \in \bar{\xi}\right\}
$$

ou seja, $\hat{\mathcal{C}}\left(\left\{\mathbb{R}^{d} \times \mathbb{R}^{d} \times \mathcal{N}^{m}\right\} \backslash \Xi_{2}\right)=0$. Mais do que isso, a integral de uma função $\phi: \mathbb{R}^{d} \times \mathbb{R}^{d} \times \mathcal{N}^{m} \rightarrow \mathbb{R}$ em relação a $\hat{\mathcal{C}}$ é dada por

$$
\hat{\mathcal{C}}(\phi):=\int_{\Xi_{2}} \phi d \hat{\mathcal{C}}=\mathbb{E}\left[\sum_{v \in S^{\circ}} \sum_{w \in S^{\circ}} \phi\left(v, w, \bar{S}^{\mathrm{o}}\right)\right] .
$$

Seja $\mathcal{G}(\xi)$ o grafo de Delaunay determinado por $\xi \in \mathcal{N}$ e, para $v, w \in \xi$, seja $a: \Xi_{2} \rightarrow \mathbb{R}$ o campo dado por $a(v, w, \bar{\xi})=\mathbf{1}\{w \in V(v, \xi)\}$. Nestas condições, a medida $\mathcal{C}$ dada em (4.17), é simplesmente a medida absolutamente contínua em relação a $\hat{\mathcal{C}}$, com derivada de Radon-Nikodim dada por $a(v, w, \bar{\xi}) \delta_{o}(v)$, ou seja,

$$
\mathcal{C}(\zeta)=\mathbb{E}\left[\sum_{v \in S^{\circ}} a\left(0, v, \bar{S}^{\circ}\right) \zeta\left(0, v, \bar{S}^{\circ}\right)\right],
$$

onde $\zeta: \Xi_{2} \rightarrow \mathbb{R}$. 


\section{Referências Bibliográficas}

[1] F. Baccelli and B. Blaszczyszyn, On a coverage process ranging from the boolean model to the poisson-voronoi tessellation, Advances in Applied Probability 33 (2001), 293-323.

[2] M. T. Barlow, Random walks on supercritical percolation clusters, Ann. Probab. 32 (2004), no. 4, 3024-3084. MR MR2094438 (2006e:60146)

[3] V. Baumstark and G. Last, Some distributional results for poisson-voronoi tessellations, Advances in Applied Probability 39 (2007), no. 1, 16-40.

[4] N. Berger and M. Biskup, Quenched invariance principle for simple random walk on percolation clusters, Probab. Theory Rel. Fields 137 (2007), no. 1-2, 83-120.

[5] P. Calka and T. Schreiber, Limit theorems for the typical poisson-voronoi cell and the crofton cell with a large inradius, The Annals of Probability 33 (2005), no. 4, 1625-1642.

[6] D. J. Daley and D. Vere-Jones, Introduction to the theory of point processes, volume 1: Elementary theory and methods, 2nd ed., Springer-Berlin, 2003.

[7] _ Introduction to the theory of point processes, volume 2: General theory and structure, 2nd ed., Springer-Berlin, 2008.

[8] P. G. Doyle and J. L. Snell, Random walks and electric networks, 2000.

[9] R. Durrett, Probability: Theory and examples, 2nd ed. ed., Duxbury Press, 1996.

[10] P. A. Ferrari, C. Landim, and H. Thorisson, Poisson trees, succession lines and coalescing random walks, Ann. Inst. H. Poincaré Probab. Statist. 40 (2004), 141-152.

[11] P. A. Ferrari and B. M. Niederhauser, Harness processes and harmonic crystals, Stoch. Process. Appl. 116 (2006), no. 6, 939-956.

[12] P. A. Ferrari, B. M. Niederhauser, and E. A. Pechersky, Harness process and non-homogeneus crystals, J. Stat. Phys. 128 (2007), 1159-1176.

[13] P.A. Ferrari, R. Fernández, and N. L. Garcia, Perfect simulation for interacting point processes, loss networks and ising models, Stochastic Processes and their Applications 102 (2002), no. 1, 63-88. 
[14] J. M. Hammersley, Harnesses, Proc. Fifth Berkeley Sympos. Mathematical Statistics and Probability III (1966), 89-117.

[15] M. Heveling and G. Last, Characterization of palm measures via bijective point-shifts, The Annals of Probability 33 (2005), no. 5, 1698-1715.

[16] A. E. Holroyd and Y. Peres, Trees and matchings from point processes, Elect. Comm. in Probab. 8 (2003), 17-27.

[17] C. T. Hsiao, Stochastic processes with gaussian interaction of components, Z. Wahrsch. Verw. Geb. 59 (1982), 39-53.

[18] C. Kipnis and S.R.S. Varadhan, Central limit theorem for additive functionals of reversible Markov processes and applications to simple exclusions., Commun. Math. Phys. 104 (1986), 1-19 (English).

[19] A. De Masi, P. A. Ferrari, S. Goldstein, and W. D. Wick, Quenched invariance principle for simple random walk on percolation clusters, Journal of Statistical Physics 55 (1989), no. 3-4, $787-855$.

[20] P. Mathieu and A. Piatinitski, Quenched invariance principle for simple random walks on percolation clusters, Proceedings A of the Royal Society 463 (2007), 2287-2307.

[21] J. Møller, Random tessellations in $\mathbb{R}^{d}$, Advances in Applied Probability 21 (1989), 37-73.

[22] J. Møller and R. P. Waagepetersen, Statistical inference and simulation for spatial point processes, Chapman \& Hall/CRC, Boca Raton, 2004.

[23] L. Muche, The poisson-voronoi tessellation: relationships for edges, Advances in Applied Probability 37 (2005), no. 2, 279-296.

[24] A. Okabe, B. Boots, K. Sugihara, and S. N. Siu, Voronoi tesselation: Concept and applications on voronoi diagrams, 2nd ed., John Wiley \& Sons, LTD, New York, 2000.

[25] P. M. Soardi, Potential theory on infinite networks, Lecture Notes on Mathematics, no. 1590, Springer-Verlag Berlin Heideberg, New York, 1994.

[26] A. A. Tempel'man, Ergodic theorems for general dynamical systems, Trans. Moscow Math. Soc. (1972), no. 26, 94-132.

[27] H. Thorisson, Coupling, stationarity and regenaration, Springer, New York, 2000.

[28] Á. Timár, Tree and grid factors for general point processes, Elect. Comm. in Probab. 9 (2004), $53-59$.

[29] H. Zessin, Point processes in general position, Journal of Contemporary Mathematical Analysis (Armenian Academy of Sciences) 43 (2008), no. 1, 59-65. 